\title{
Refolding Foldamers: Triazene-Arylene Oligomers that Change Shape with Chemical Stimuli - Supporting Information
}

\author{
Simin Liu, Peter Y. Zavalij, Yiu-Fai Lam, and Lyle Isaacs* \\ Department of Chemistry and Biochemistry, University of Maryland College Park, MD 20742
}

Table of Contents

General experimental details

Expanded view of the $\mathrm{x}$-ray crystal structures of $\mathrm{CB}[10] \cdot \mathbf{3}$,

$\mathrm{CB}[10] \bullet 4, \mathrm{CB}[10] \bullet 7$, and $\mathrm{CB}[7] \bullet 7 \bullet \mathrm{CB}[7]$

${ }^{1} \mathrm{H}$ NMR spectra for $3,4,7,8$, and 11 in $\mathrm{D}_{2} \mathrm{O}$

Additional NMR experiments for $\mathrm{CB}[\mathrm{n}] \cdot \mathbf{3}$

Additional NMR experiments for $\mathrm{CB}[\mathrm{n}] \bullet 4$

Additional NMR experiments for $\mathrm{CB}[\mathrm{n}] \cdot 7$

Additional NMR experiments for $\mathrm{CB}[\mathrm{n}] \bullet \mathbf{8}$

Additional NMR experiments for $\mathrm{CB}[\mathrm{n}] \bullet \mathbf{1 1}$

NMR control experiments for the refolding of

3 in response to chemical stimuli

${ }^{1} \mathrm{H}$ and ${ }^{13} \mathrm{C}$ NMR spectra of $\mathbf{1}-\mathbf{1 1}$ in DMSO

\section{Pages}

S2

S3 - S6

$\mathrm{S} 7-\mathrm{S} 11$

$\mathrm{S} 12-\mathrm{S} 25$

S26 - S27

$\mathrm{S} 28-\mathrm{S} 36$

$\mathrm{S} 37-\mathrm{S} 40$

$\mathrm{S} 41-\mathrm{S} 45$

S46 - S54

S55 - S76 
General Experimental. Starting materials were purchased from commercial suppliers and were used without further purification. Melting points were measured in open capillary tubes and are uncorrected. NMR spectra were measured on spectrometers operating at 400 or 500 $\mathrm{MHz}$ for ${ }^{1} \mathrm{H}$ and 100 or $125 \mathrm{MHz}$ for ${ }^{13} \mathrm{C}$. The NMR temperature was maintained $( \pm 0.5 \mathrm{~K})$ with a Bruker eurotherm module that had been calibrated using the separation of the resonances of methanol. All spectra are referenced relative to residual ${ }^{1} \mathrm{H}$ solvent resonances. Mass spectrometry was performed using a JEOL AccuTOF electrospray mass spectrometer. 


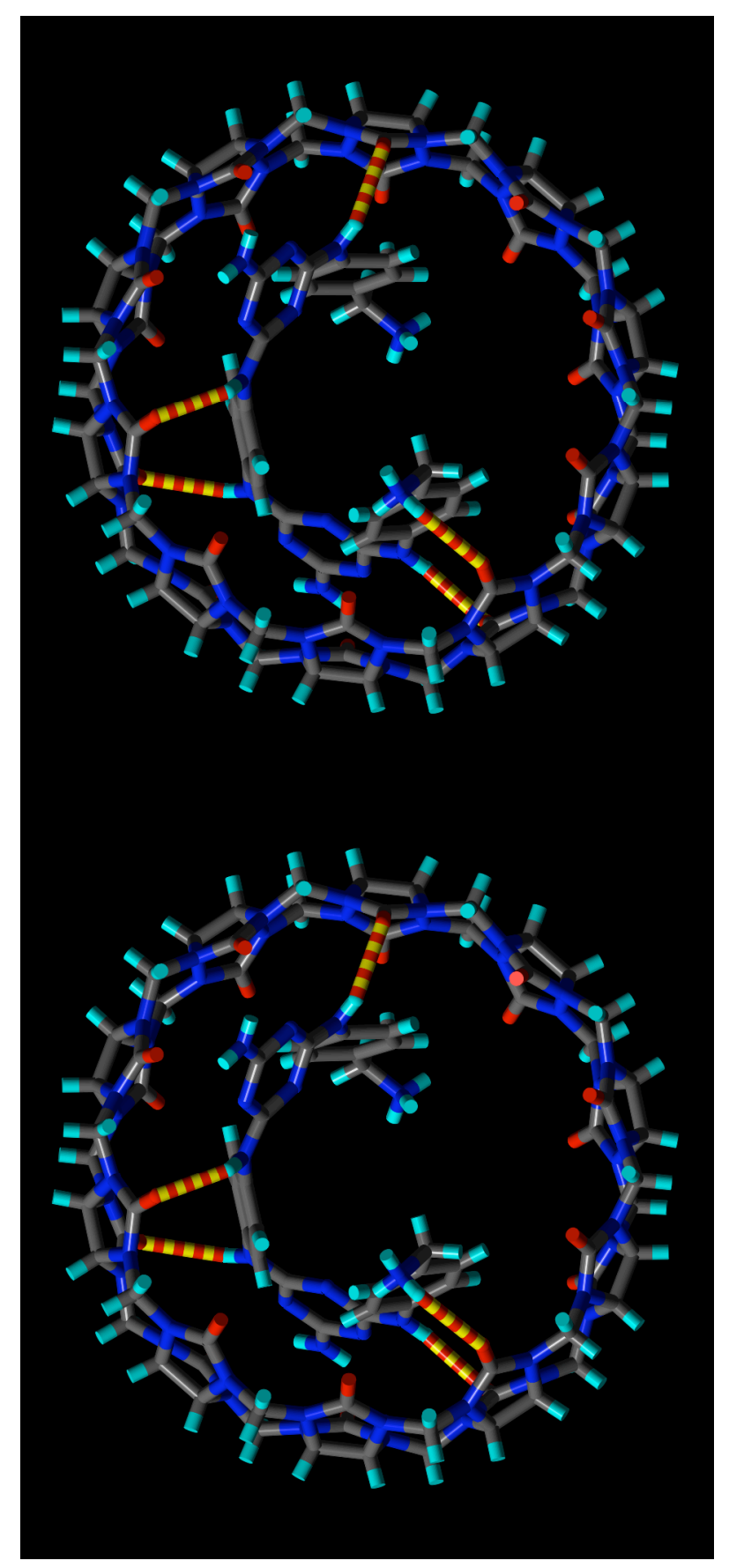

Figure S1. Enlarged cross-eyed stereoview of the structure of $\mathrm{CB}[10] \bullet 4$ presented in the text. Color code: $\mathrm{C}$, gray; $\mathrm{H}$, aqua; N, blue; O, red; H-bonds, red-yellow striped. 


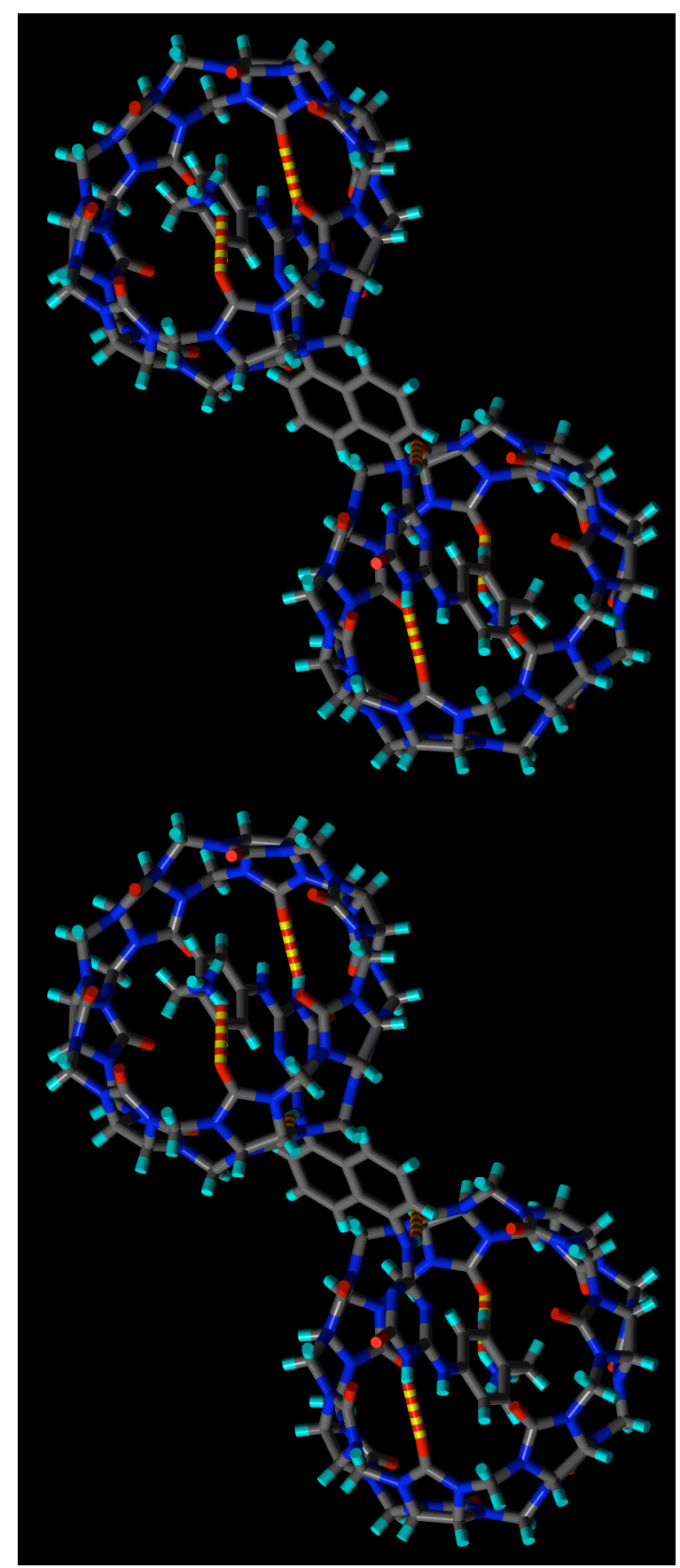

Figure S2. Enlarged cross-eyed stereoview of the structure of $\mathrm{CB}[7] \cdot 7 \cdot \mathrm{CB}[7]$ presented in the text. Color code: $\mathrm{C}$, gray; H, aqua; N, blue; O, red; H-bonds, red-yellow striped. 


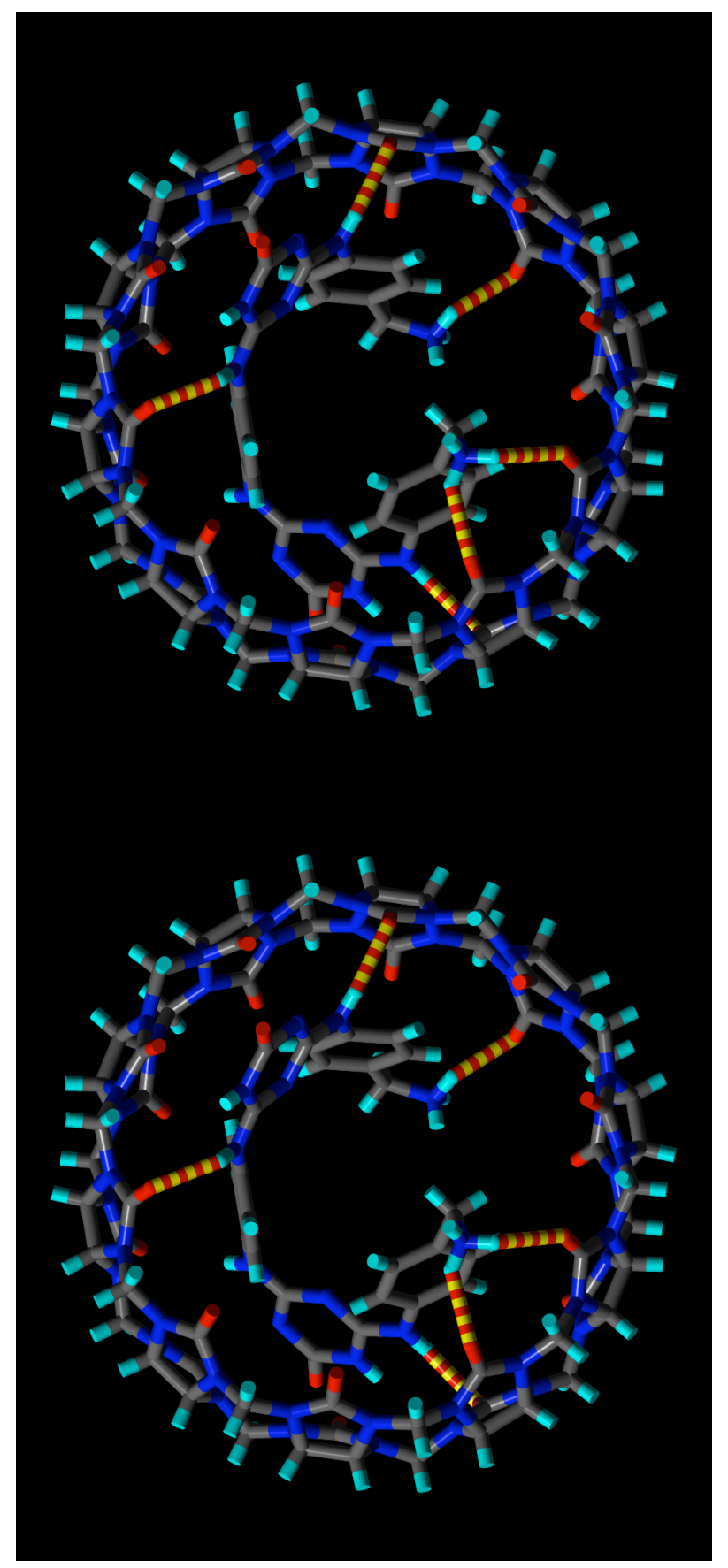

Figure S3. Enlarged cross-eyed stereoview of the structure of $\mathrm{CB}[10] \bullet \mathbf{3}$ presented in the text. Color code: $\mathrm{C}$, gray; $\mathrm{H}$, aqua; $\mathrm{N}$, blue; $\mathrm{O}$, red; H-bonds, red-yellow striped. 


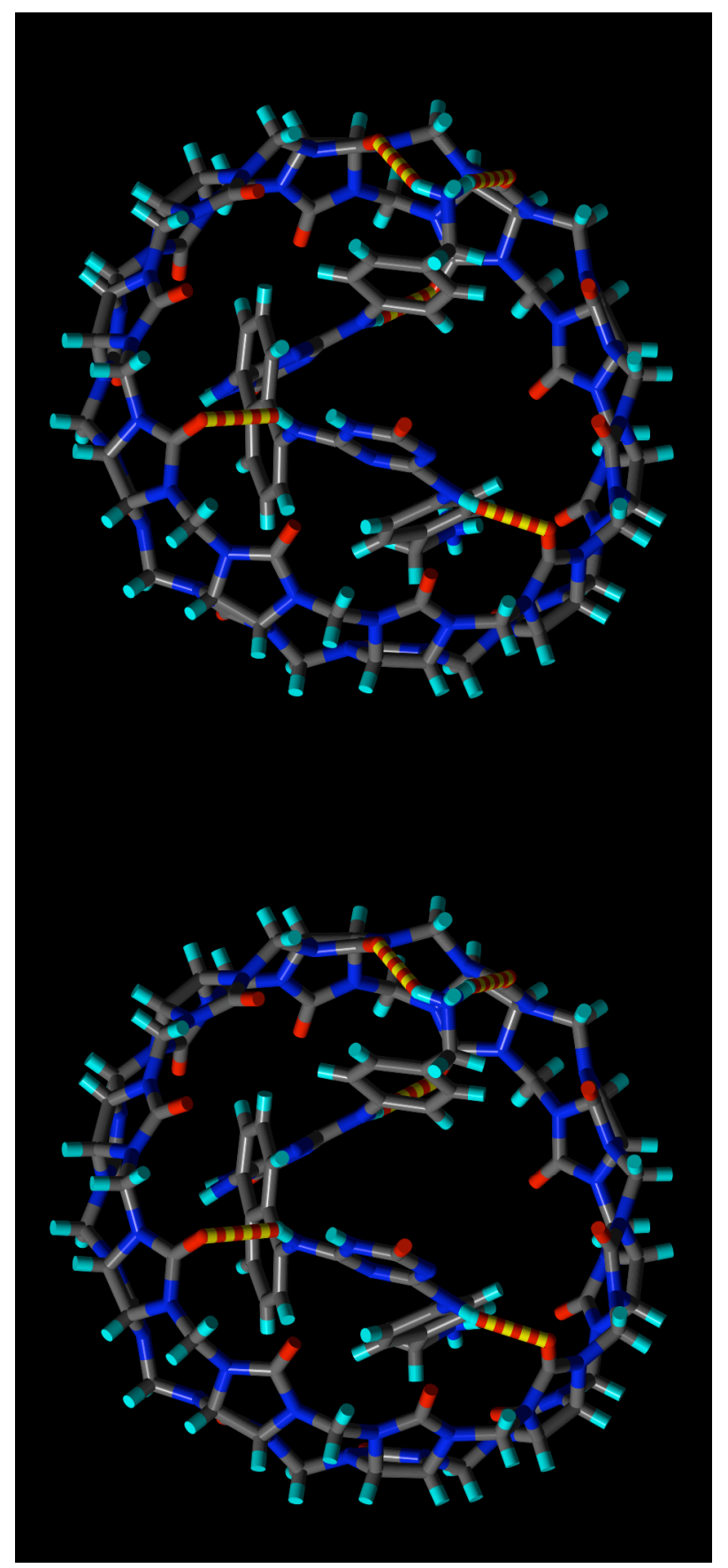

Figure S4. Enlarged cross-eyed stereoview of the structure of $\mathrm{CB}[10] \cdot 7$ presented in the text. Color code: $\mathrm{C}$, gray; $\mathrm{H}$, aqua; $\mathrm{N}$, blue; $\mathrm{O}$, red; H-bonds, red-yellow striped. 


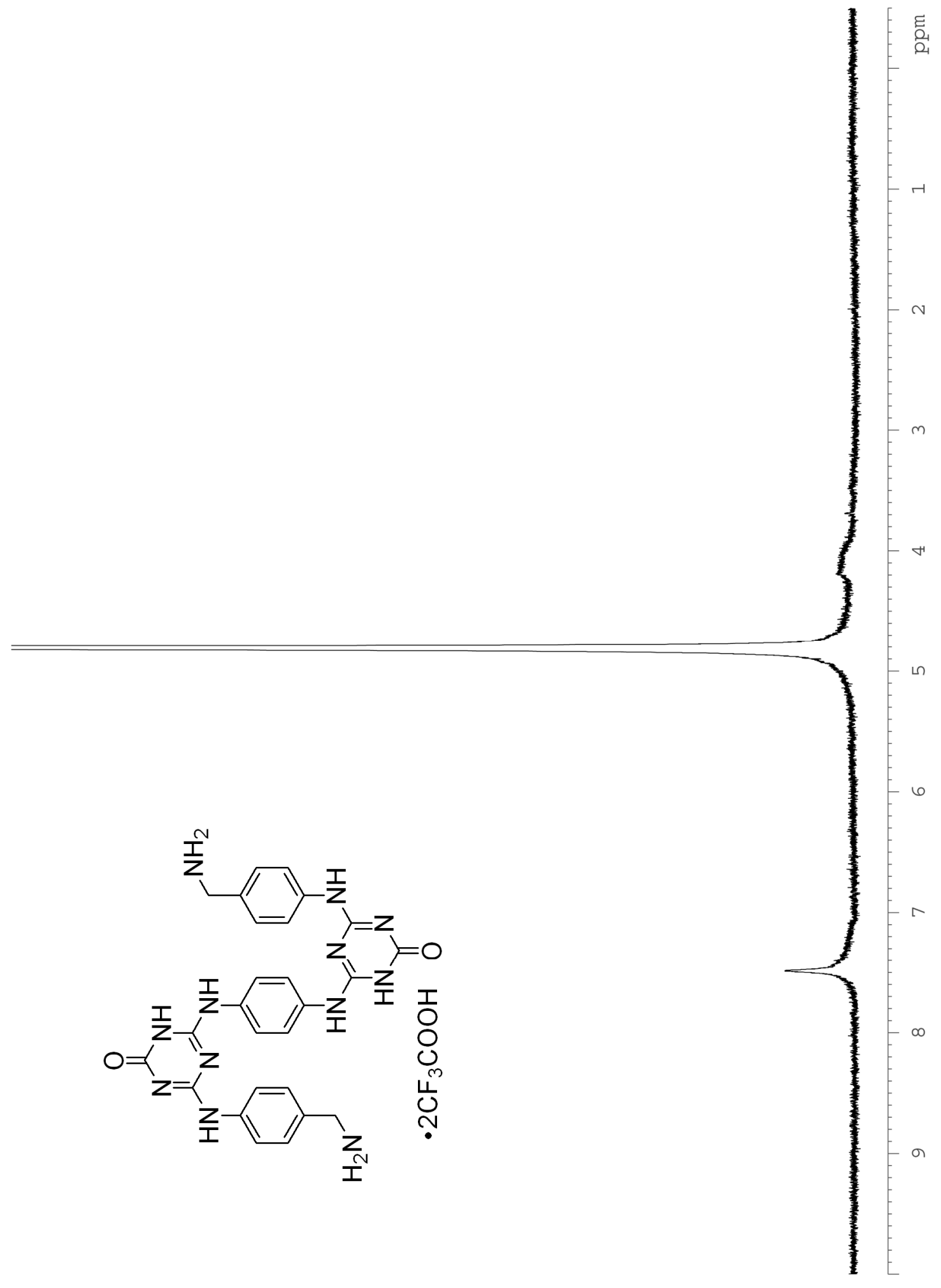

Figure S5. ${ }^{1} \mathrm{H}$ NMR spectrum $\left(400 \mathrm{MHz}, \mathrm{D}_{2} \mathrm{O}, \mathrm{RT}\right)$ for 3. 


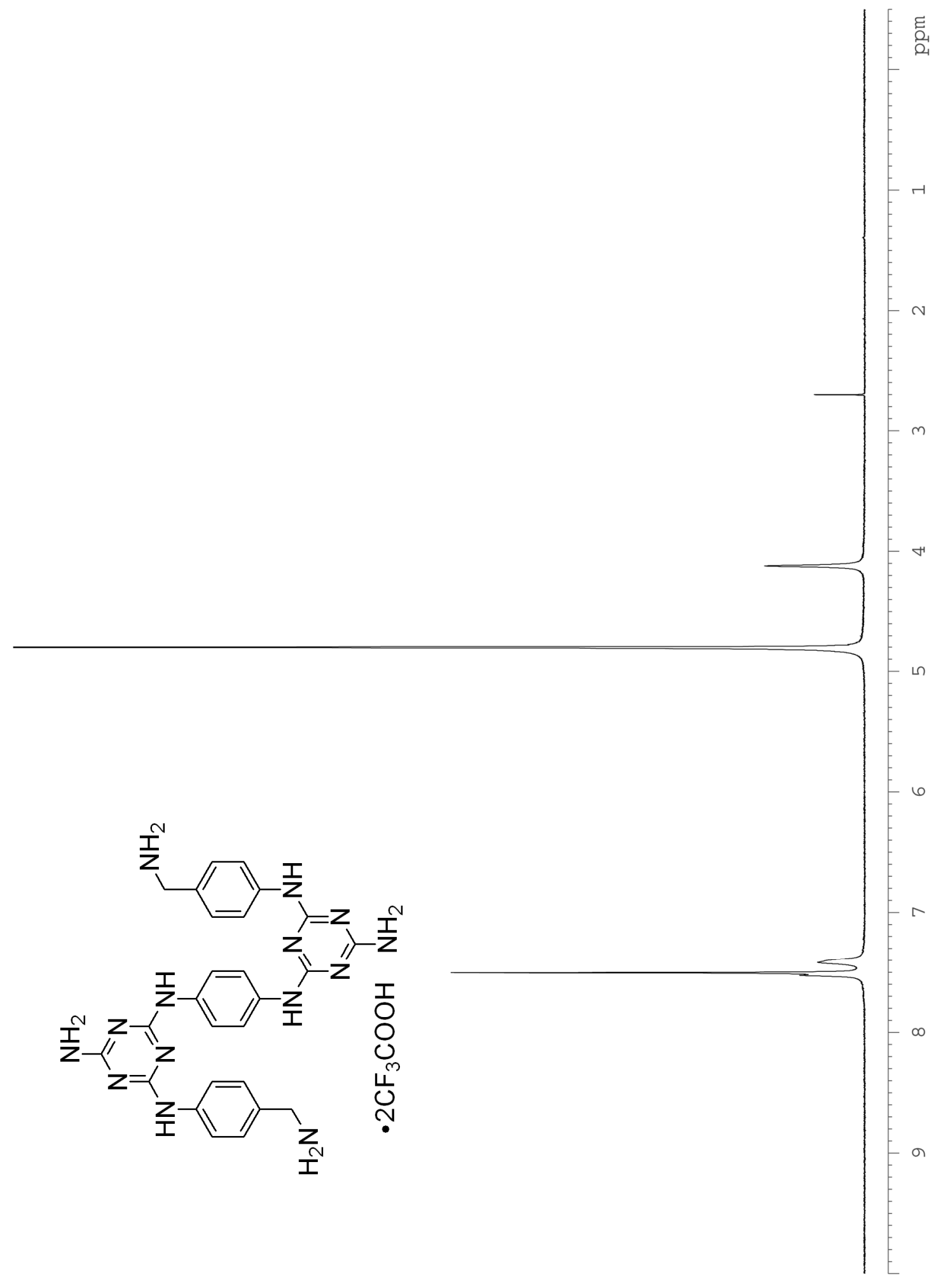

Figure S6. ${ }^{1} \mathrm{H}$ NMR spectrum $\left(400 \mathrm{MHz}, \mathrm{D}_{2} \mathrm{O}, \mathrm{RT}\right)$ for 4. 


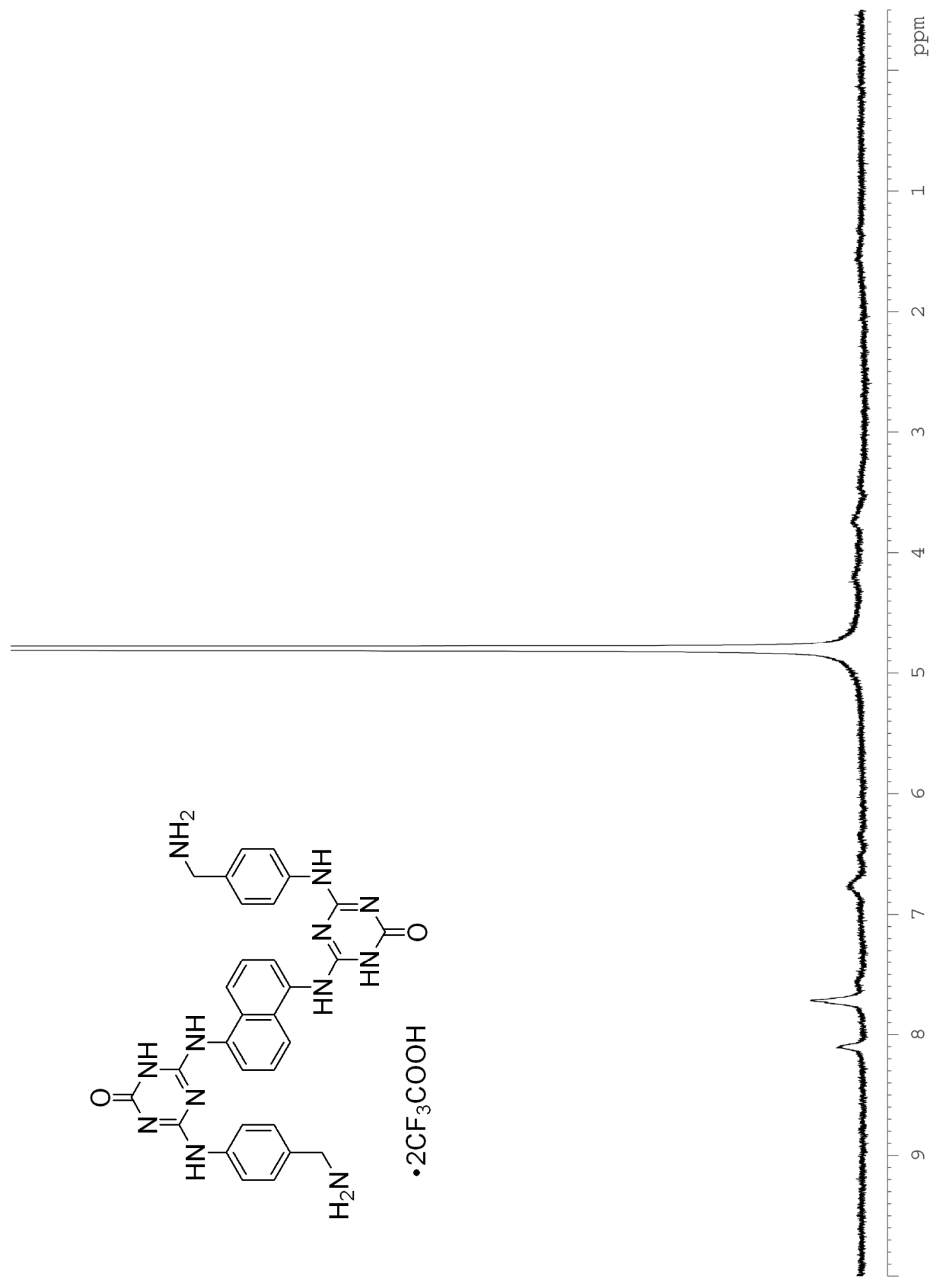

Figure S7. ${ }^{1} \mathrm{H}$ NMR spectrum $\left(400 \mathrm{MHz}, \mathrm{D}_{2} \mathrm{O}, \mathrm{RT}\right)$ for 7. 


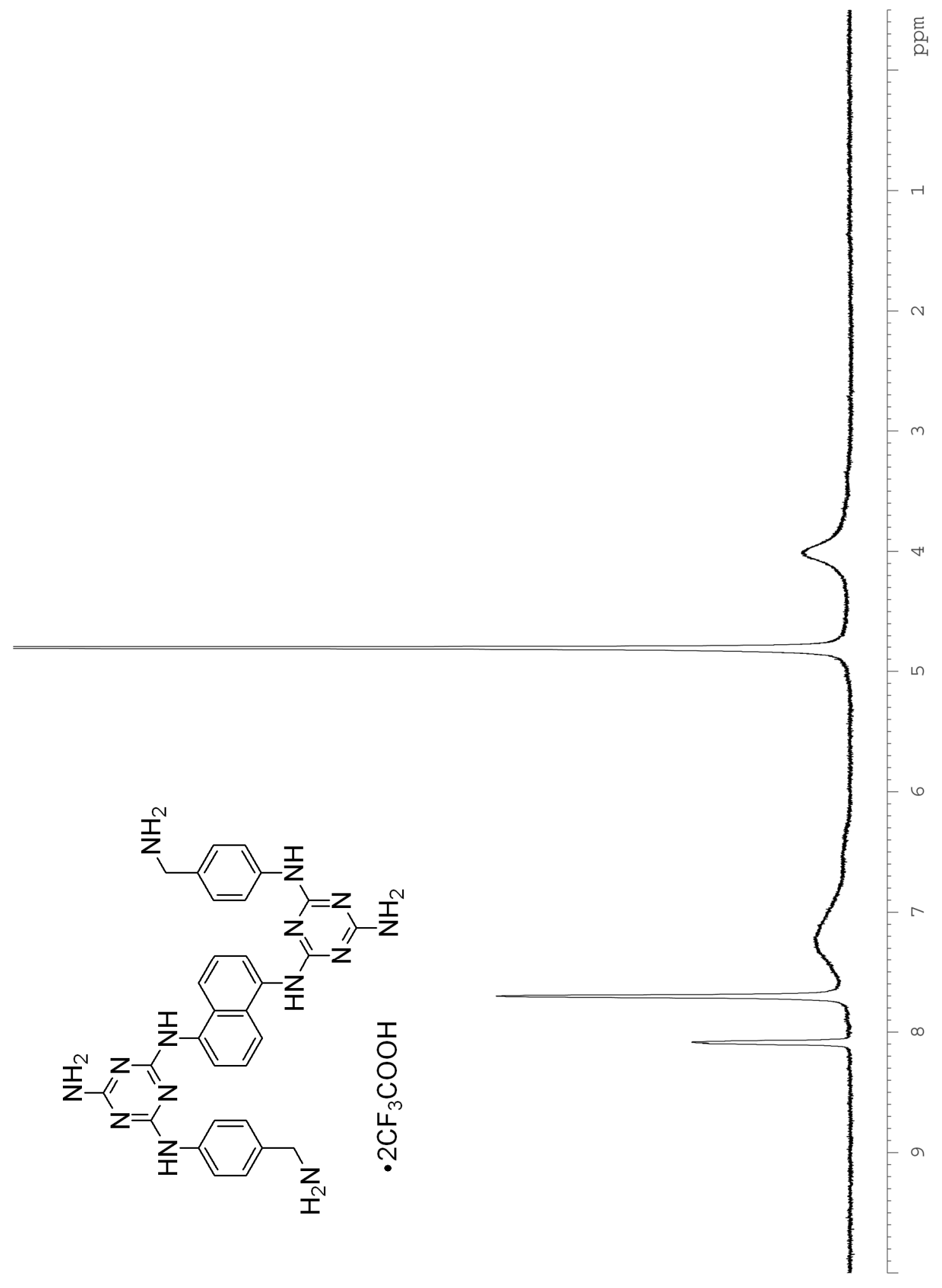

Figure S8. ${ }^{1} \mathrm{H}$ NMR spectrum $\left(400 \mathrm{MHz}, \mathrm{D}_{2} \mathrm{O}, \mathrm{RT}\right)$ for 8. 


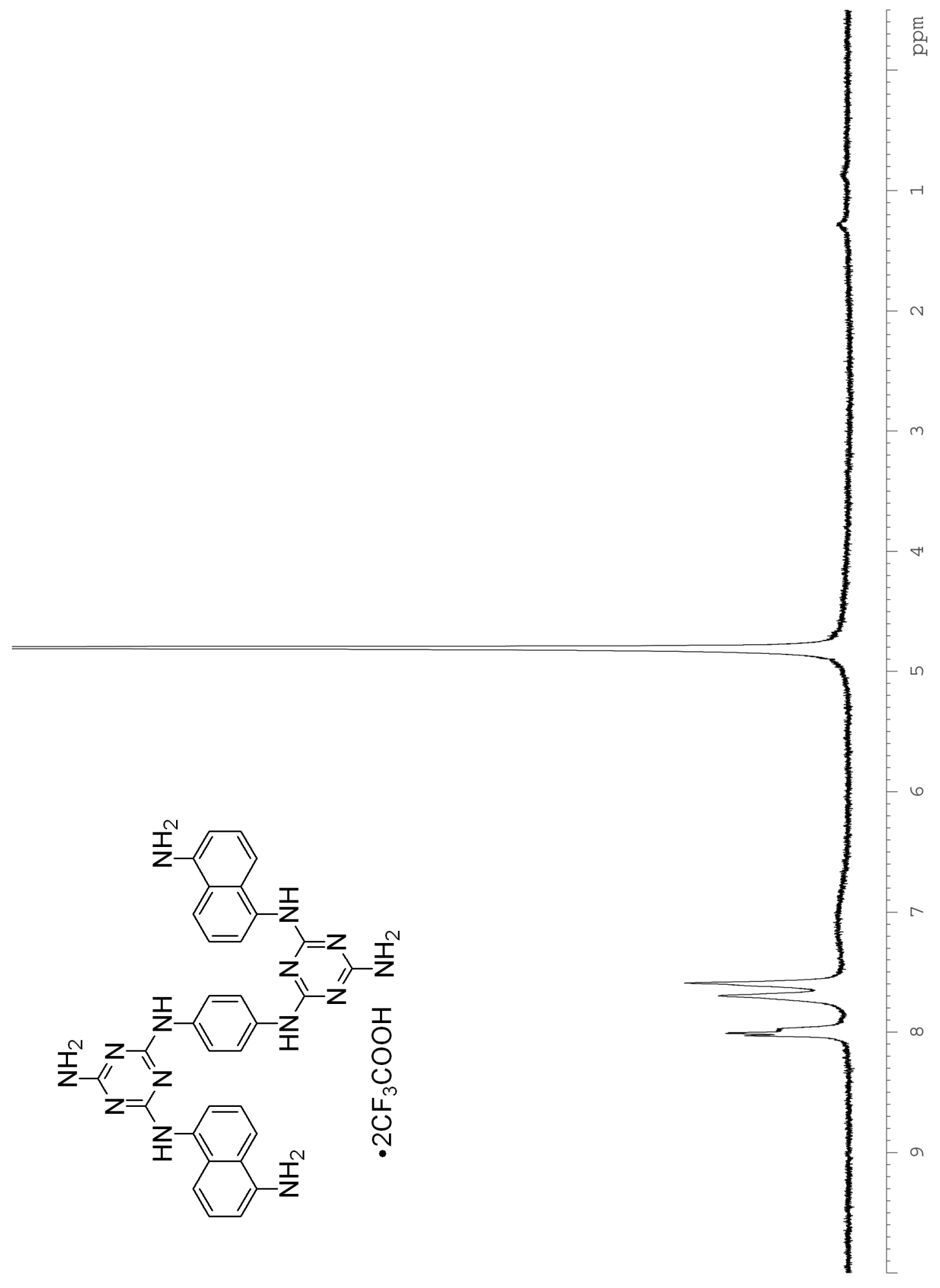

Figure S9. ${ }^{1} \mathrm{H}$ NMR spectrum (400 MHz, $\left.\mathrm{D}_{2} \mathrm{O}, \mathrm{RT}\right)$ for 11. 


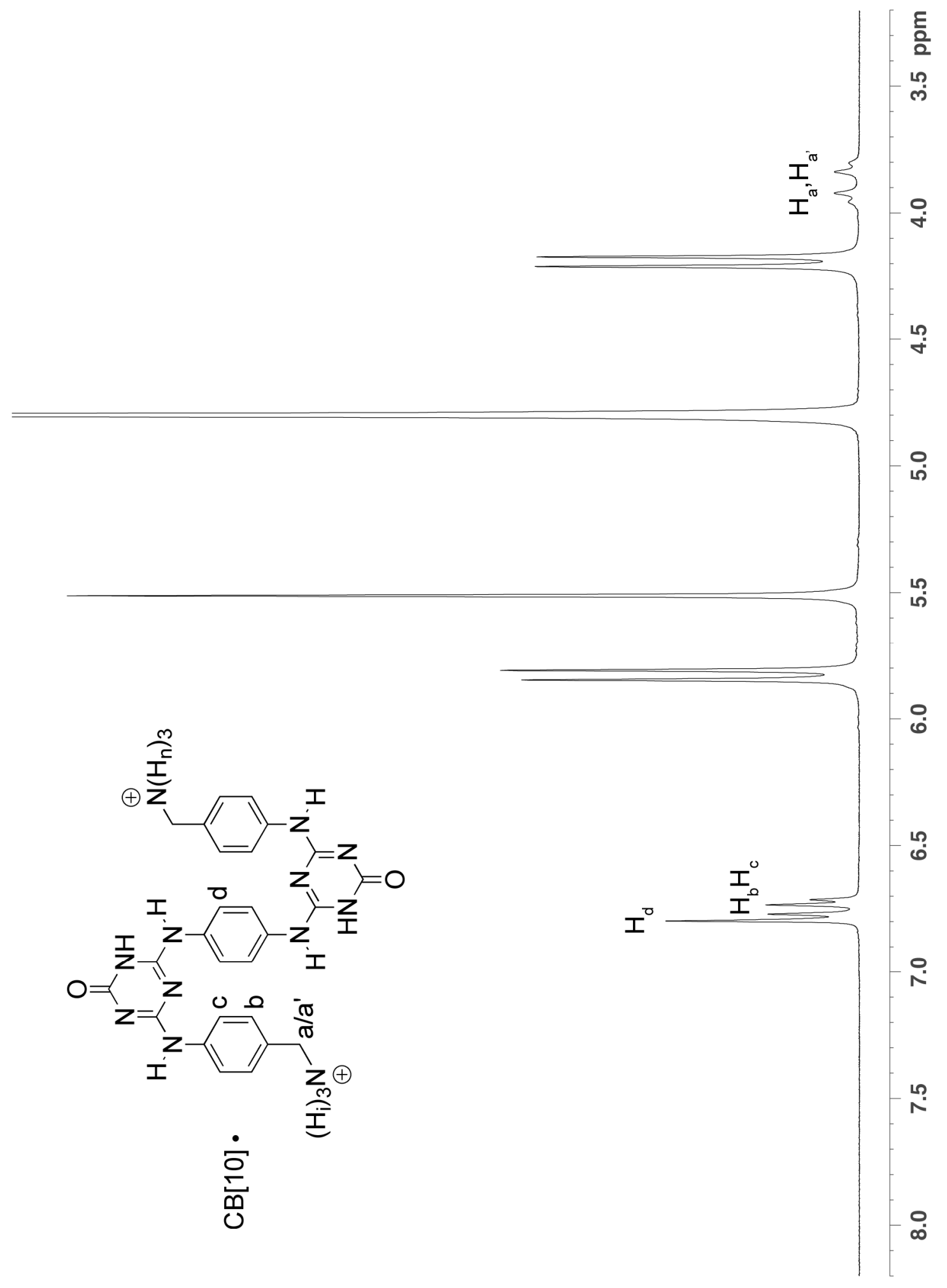

Figure S10. ${ }^{1} \mathrm{H}$ NMR spectrum $\left(400 \mathrm{MHz}, \mathrm{D}_{2} \mathrm{O}, \mathrm{RT}\right)$ for $\mathrm{CB}[10] \bullet 3$. 


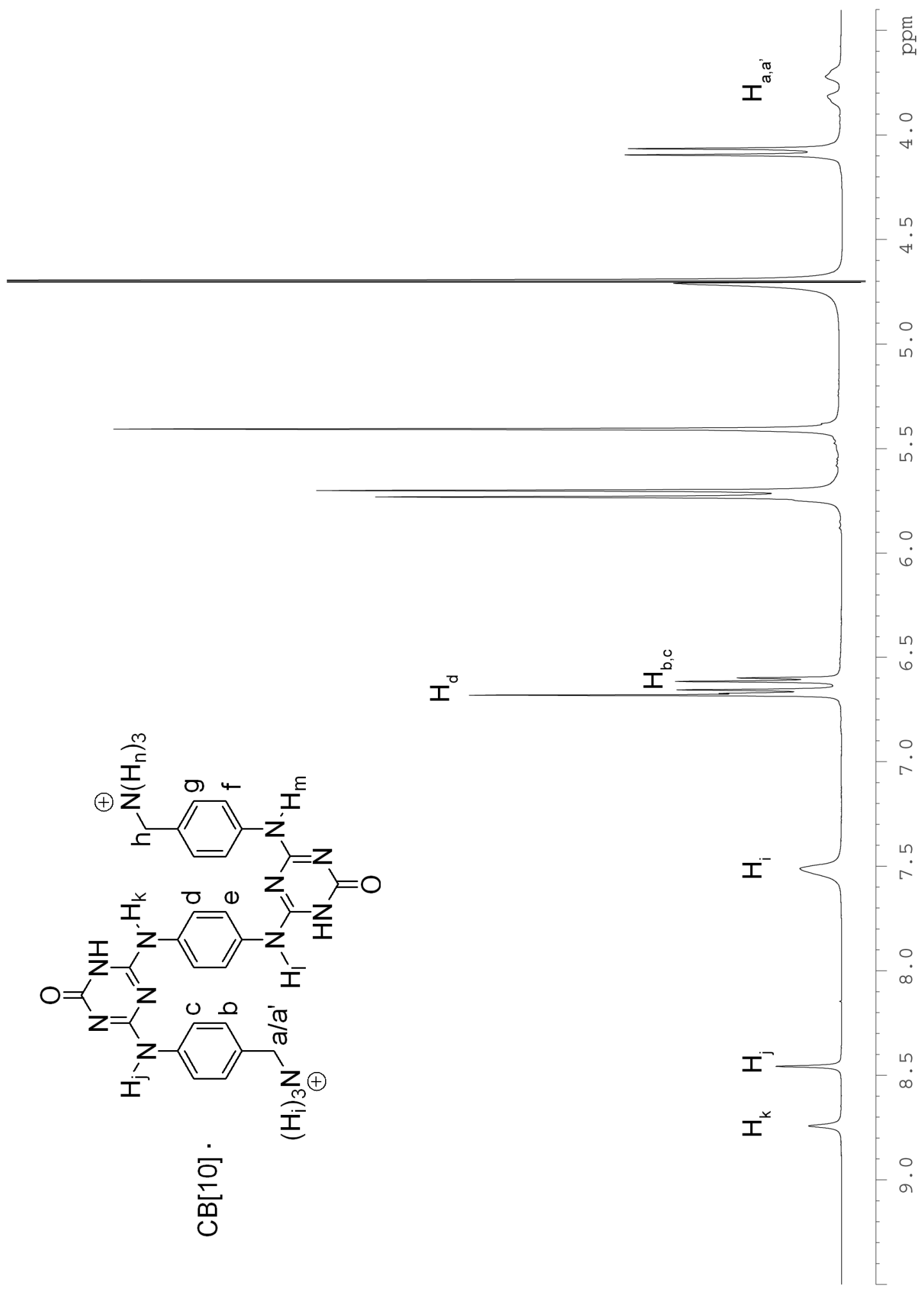

Figure S11. ${ }^{1} \mathrm{H}$ NMR spectrum $\left(500 \mathrm{MHz}, 85 \% \mathrm{H}_{2} \mathrm{O} / \mathrm{D}_{2} \mathrm{O}, \mathrm{RT}\right)$ for $\mathrm{CB}[10] \bullet 3$. 


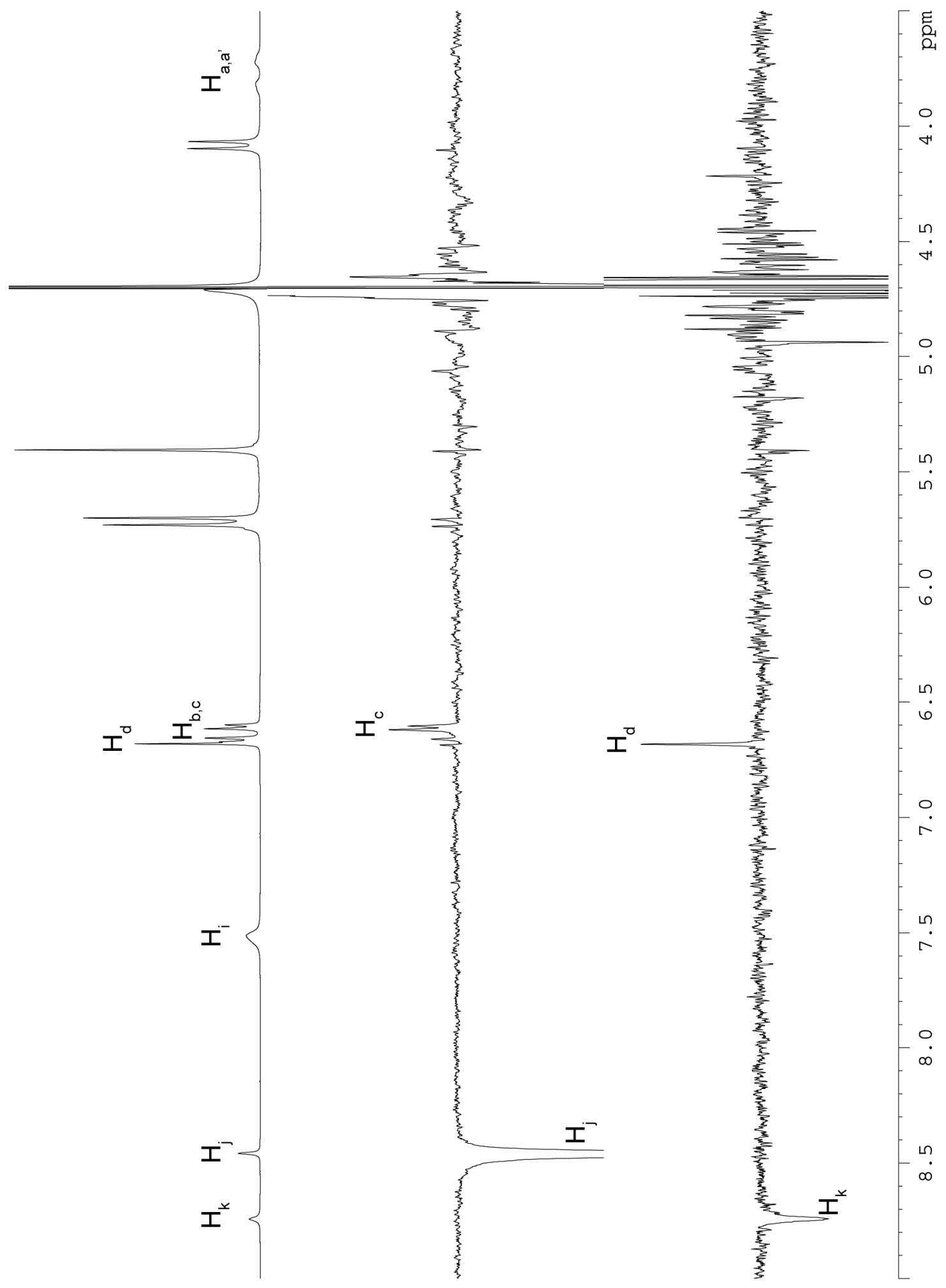

Figure S12. Selective 1D ROE spectra $\left(500 \mathrm{MHz}, 85 \% \mathrm{H}_{2} \mathrm{O} / \mathrm{D}_{2} \mathrm{O}, \mathrm{RT}\right)$ for $\mathrm{CB}[10] \bullet 3$. 


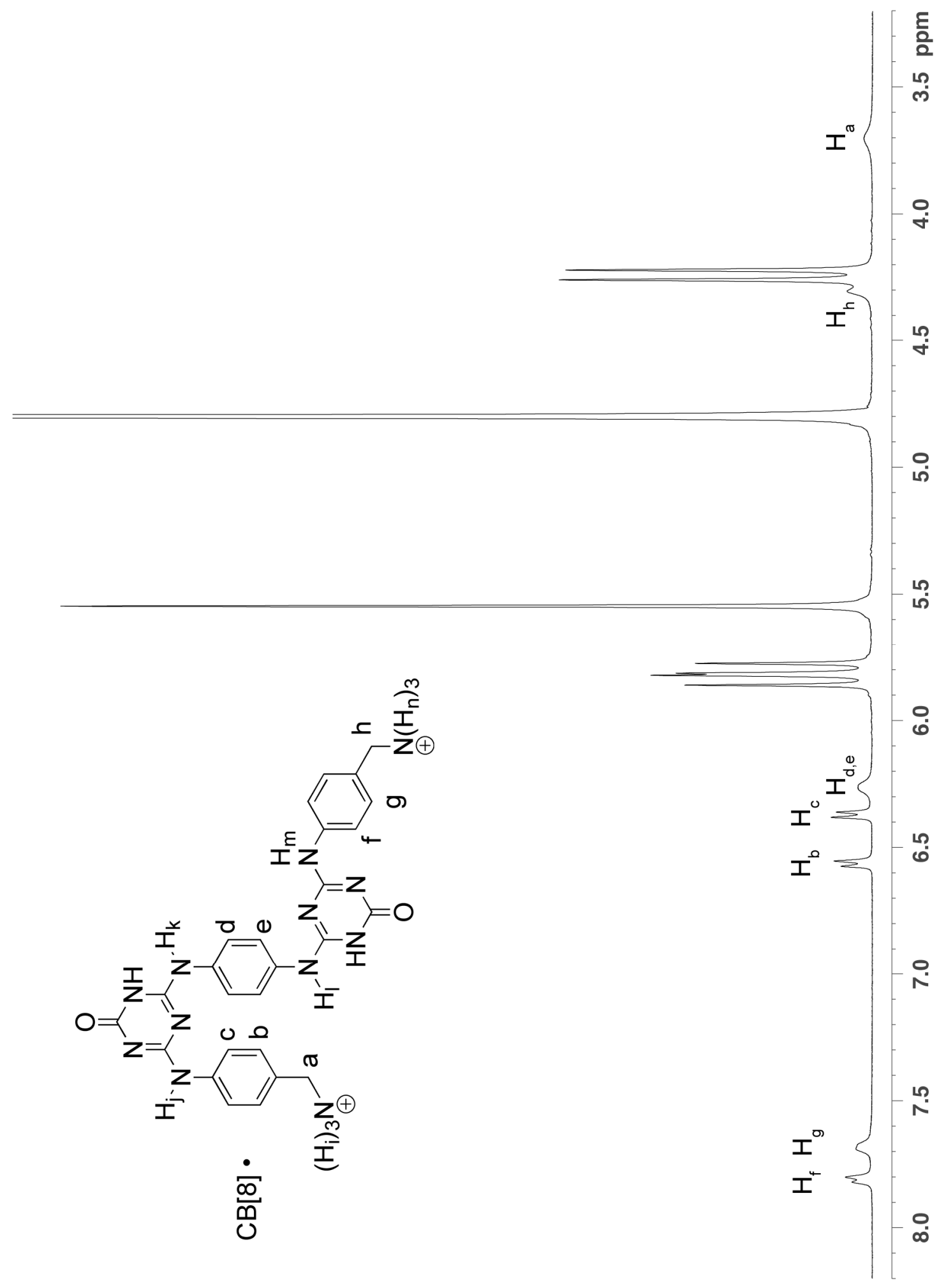

Figure S13. ${ }^{1} \mathrm{H}$ NMR spectrum $\left(400 \mathrm{MHz}, \mathrm{D}_{2} \mathrm{O}, \mathrm{RT}\right)$ for $\mathrm{CB}[8] \bullet 3$. 


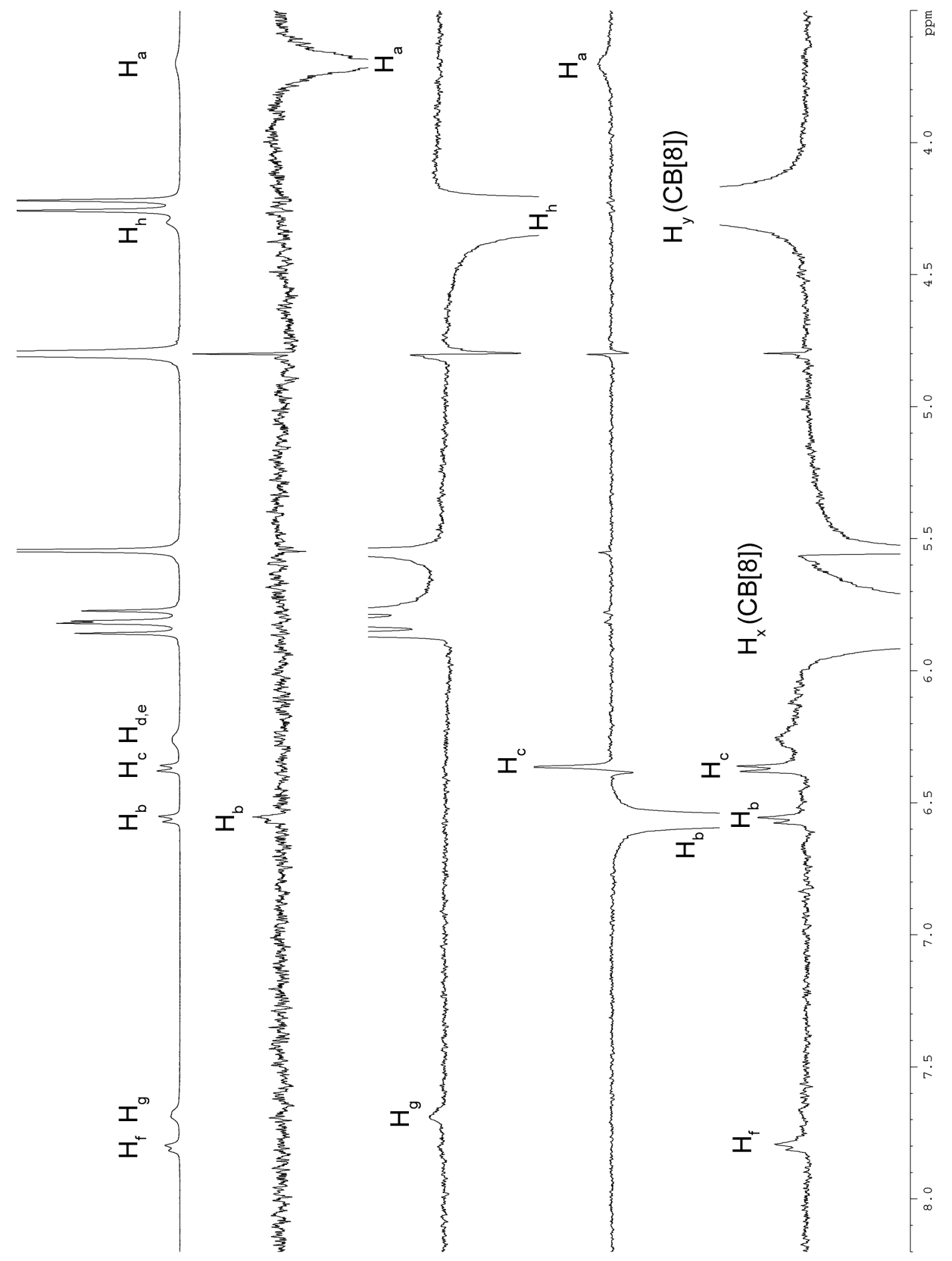

Figure S14. Selective 1D ROE spectra (400 MHz, $\left.\mathrm{D}_{2} \mathrm{O}, \mathrm{RT}\right)$ for $\mathrm{CB}[8] \bullet \mathbf{3}$. 


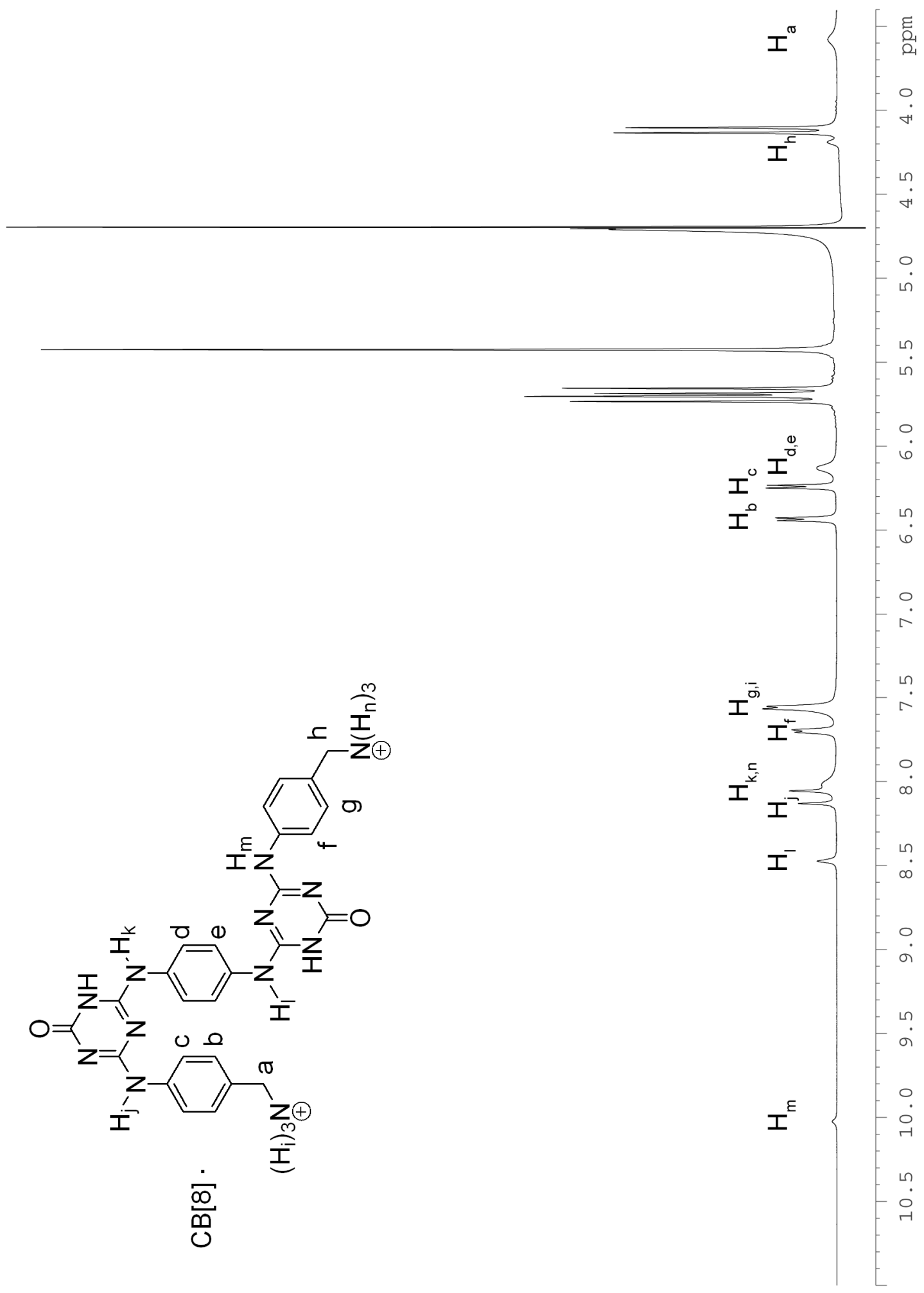

Figure S15. ${ }^{1} \mathrm{H}$ NMR spectrum $\left(500 \mathrm{MHz}, 85 \% \mathrm{H}_{2} \mathrm{O} / \mathrm{D}_{2} \mathrm{O}, \mathrm{RT}\right)$ for $\mathrm{CB}[8] \bullet 3$. 


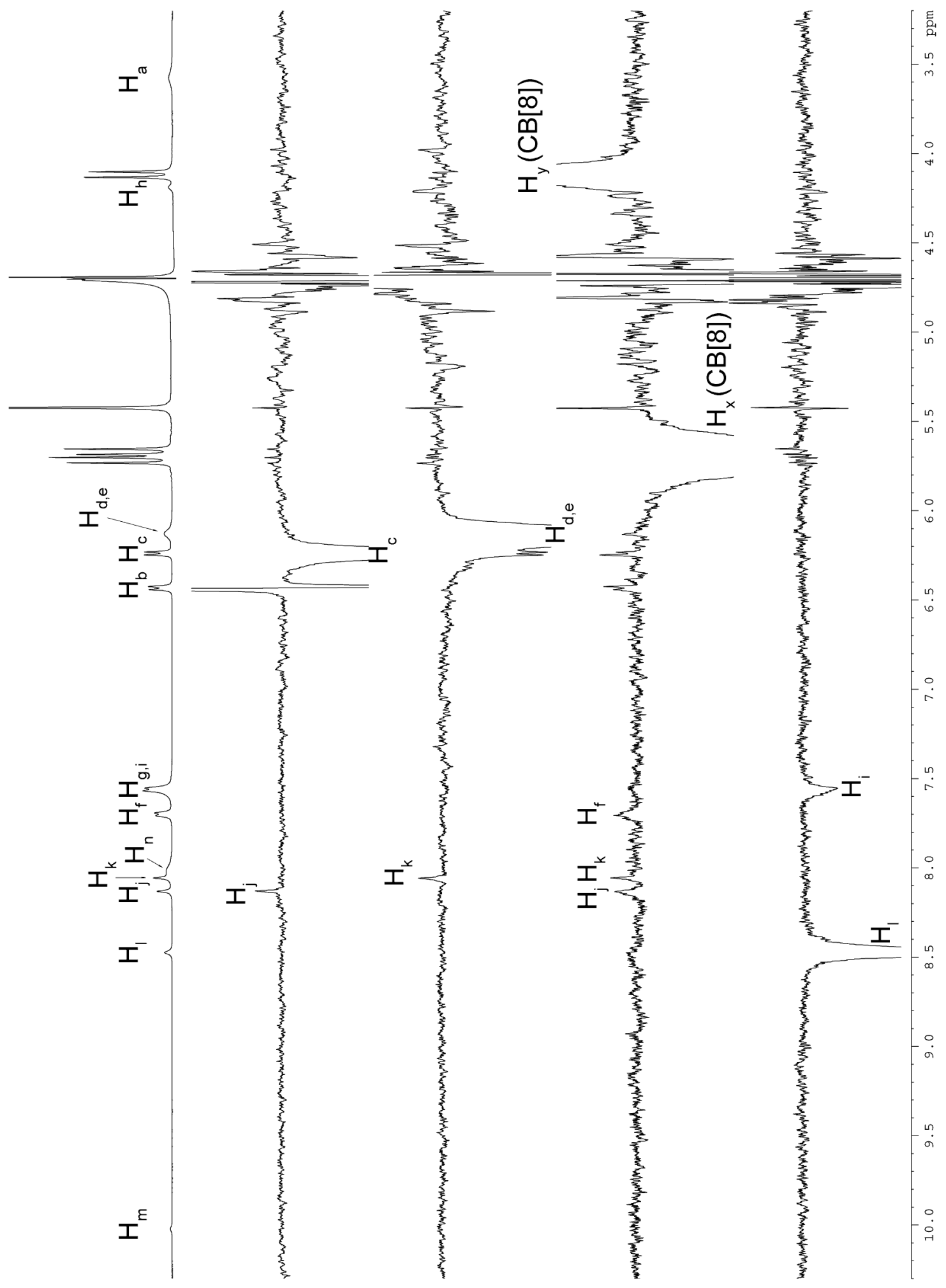

Figure S16. Selective 1D ROE spectra $\left(500 \mathrm{MHz}, 85 \% \mathrm{H}_{2} \mathrm{O} / \mathrm{D}_{2} \mathrm{O}\right.$, RT) for $\mathrm{CB}[8] \bullet 3$. 


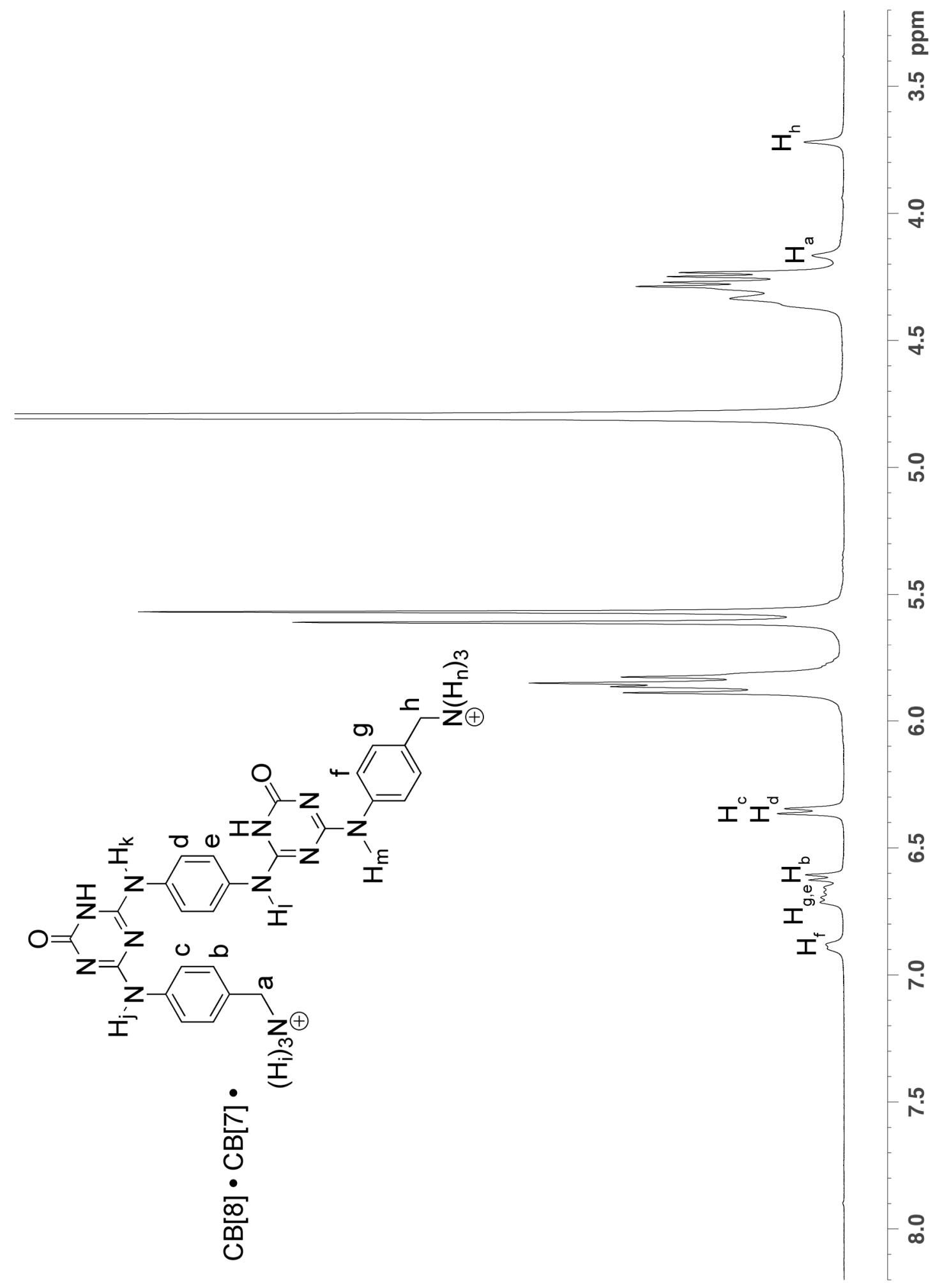

Figure S17. ${ }^{1} \mathrm{H}$ NMR spectrum (400 $\left.\mathrm{MHz}, \mathrm{D}_{2} \mathrm{O}, \mathrm{RT}\right)$ for $\mathrm{CB}[8] \bullet 3 \bullet \mathrm{CB}[7]$. 


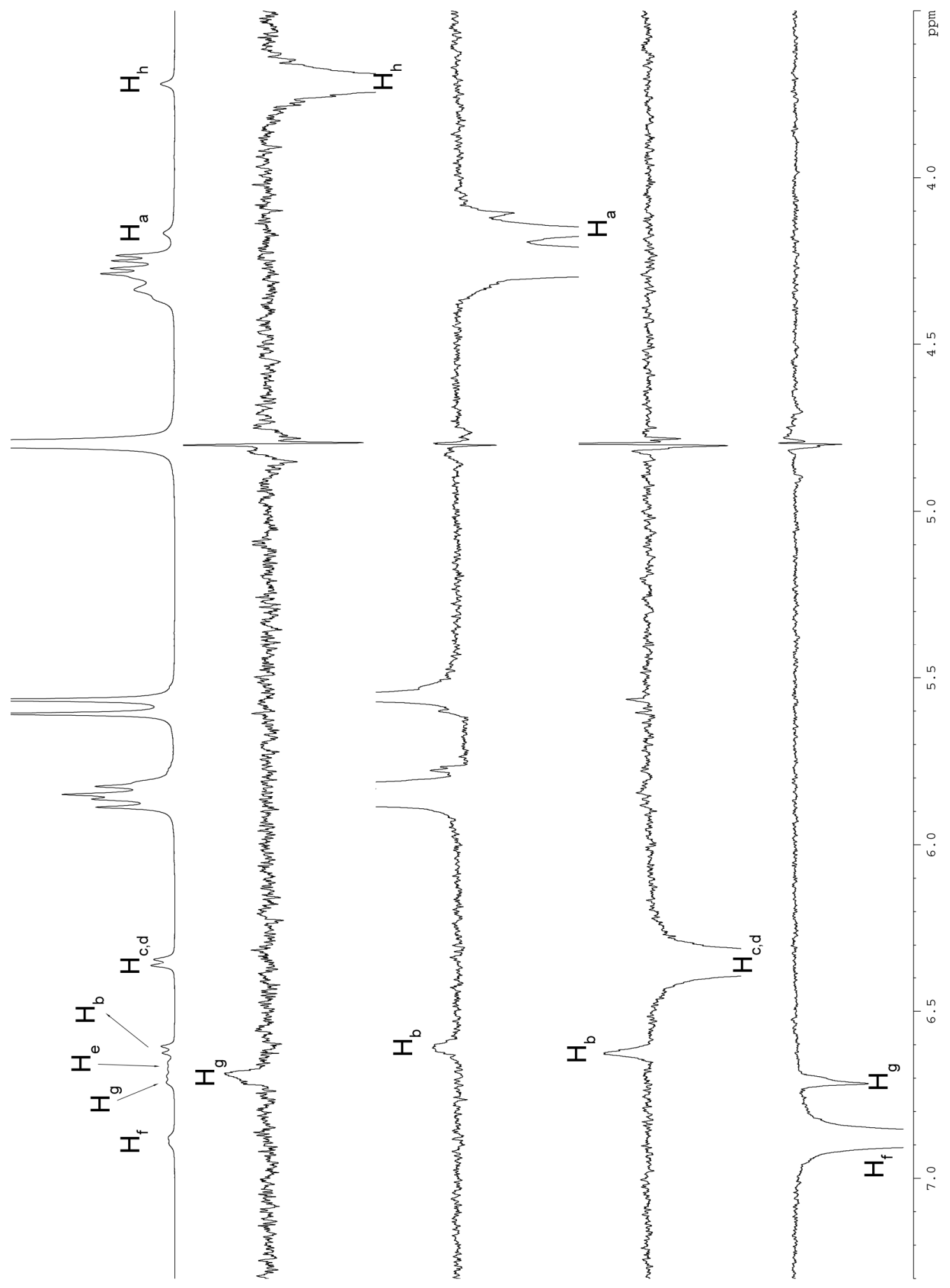

Figure S18. Selective 1D ROE spectra (400 MHz, $\left.\mathrm{D}_{2} \mathrm{O}, \mathrm{RT}\right)$ for $\mathrm{CB}[8] \bullet 3 \bullet \mathrm{CB}[7]$. 


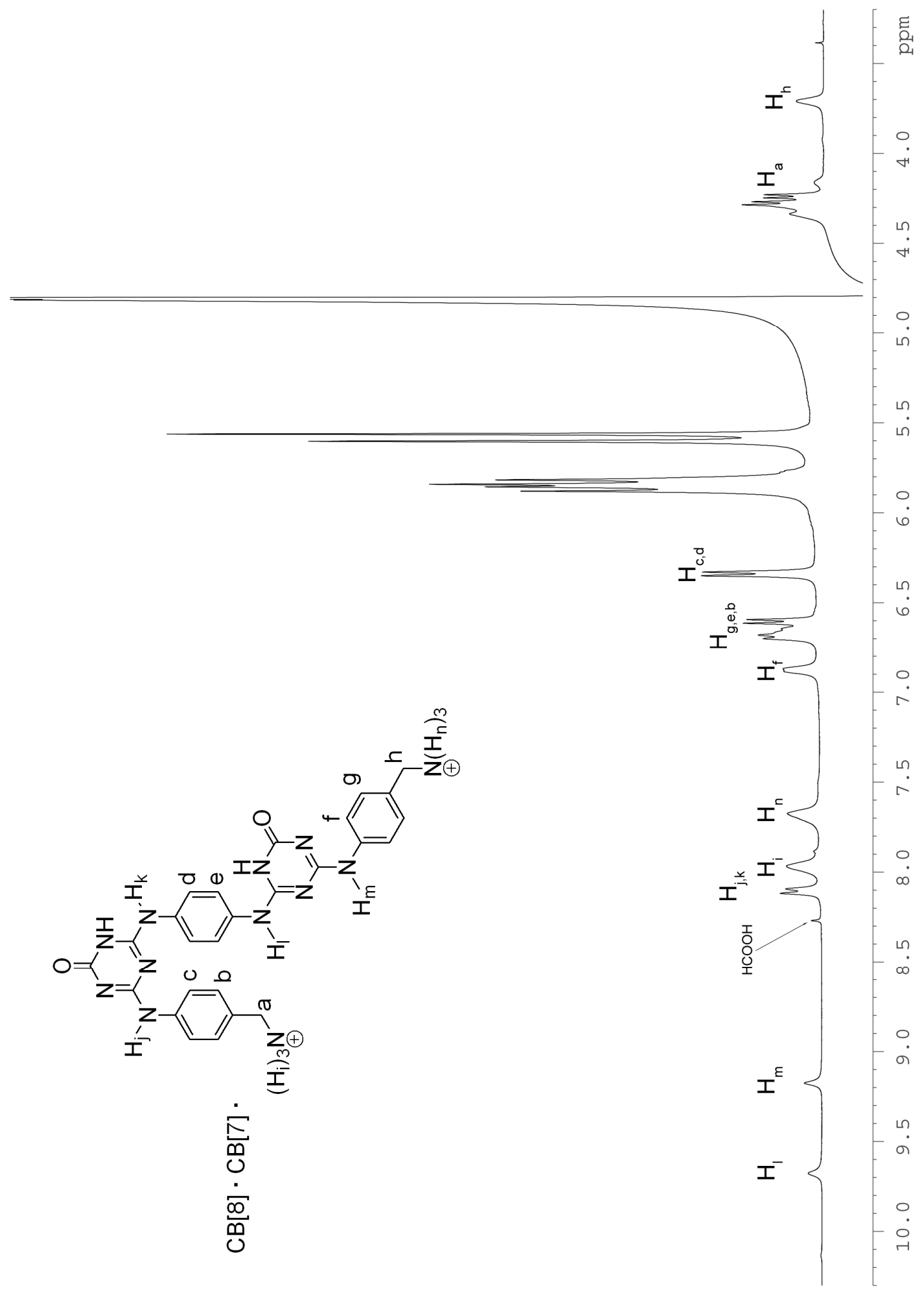

Figure S19. ${ }^{1} \mathrm{H}$ NMR spectrum $\left(400 \mathrm{MHz}, 85 \% \mathrm{H}_{2} \mathrm{O} / \mathrm{D}_{2} \mathrm{O}, \mathrm{RT}\right)$ for $\mathrm{CB}[8] \cdot 3 \bullet \mathrm{CB}[7]$. 


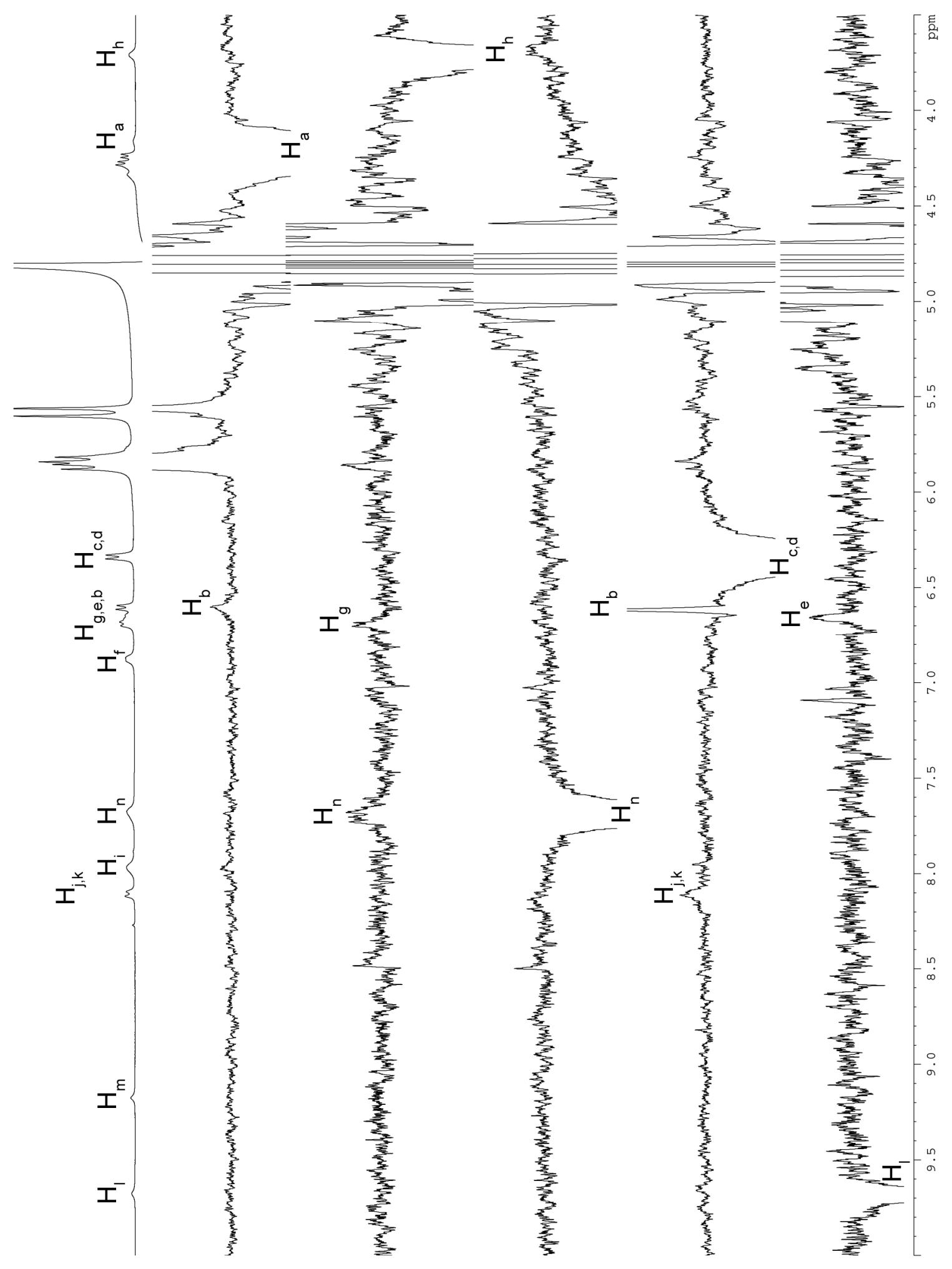

Figure S20. Selective 1D ROE spectra $\left(400 \mathrm{MHz}, 85 \% \mathrm{H}_{2} \mathrm{O} / \mathrm{D}_{2} \mathrm{O}, \mathrm{RT}\right)$ for $\mathrm{CB}[8] \cdot 3 \cdot \mathrm{CB}[7]$. 


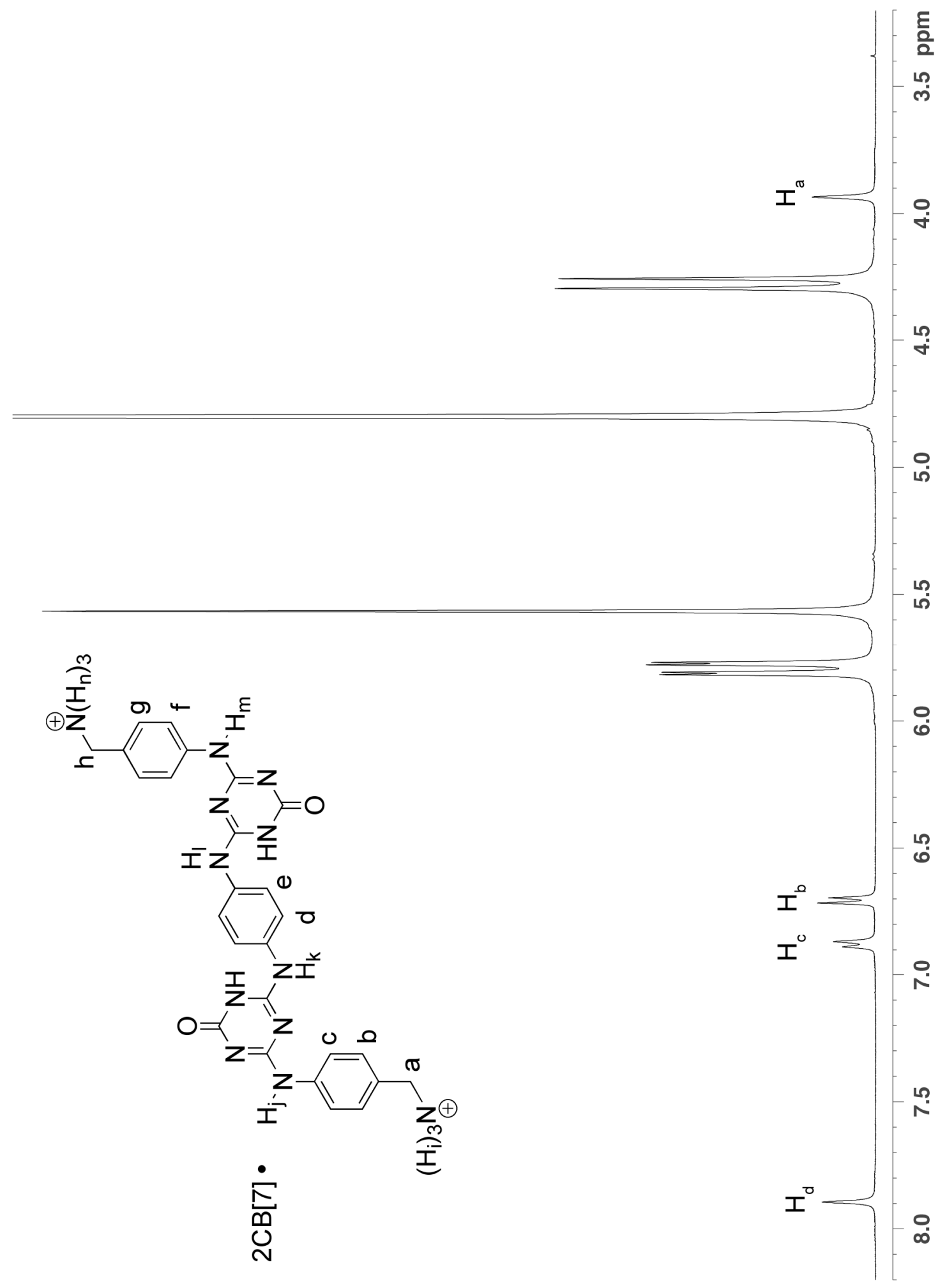

Figure S21. ${ }^{1} \mathrm{H}$ NMR spectrum $\left(400 \mathrm{MHz}, \mathrm{D}_{2} \mathrm{O}, \mathrm{RT}\right)$ for $\mathrm{CB}[7] \bullet 3 \bullet \mathrm{CB}[7]$. 


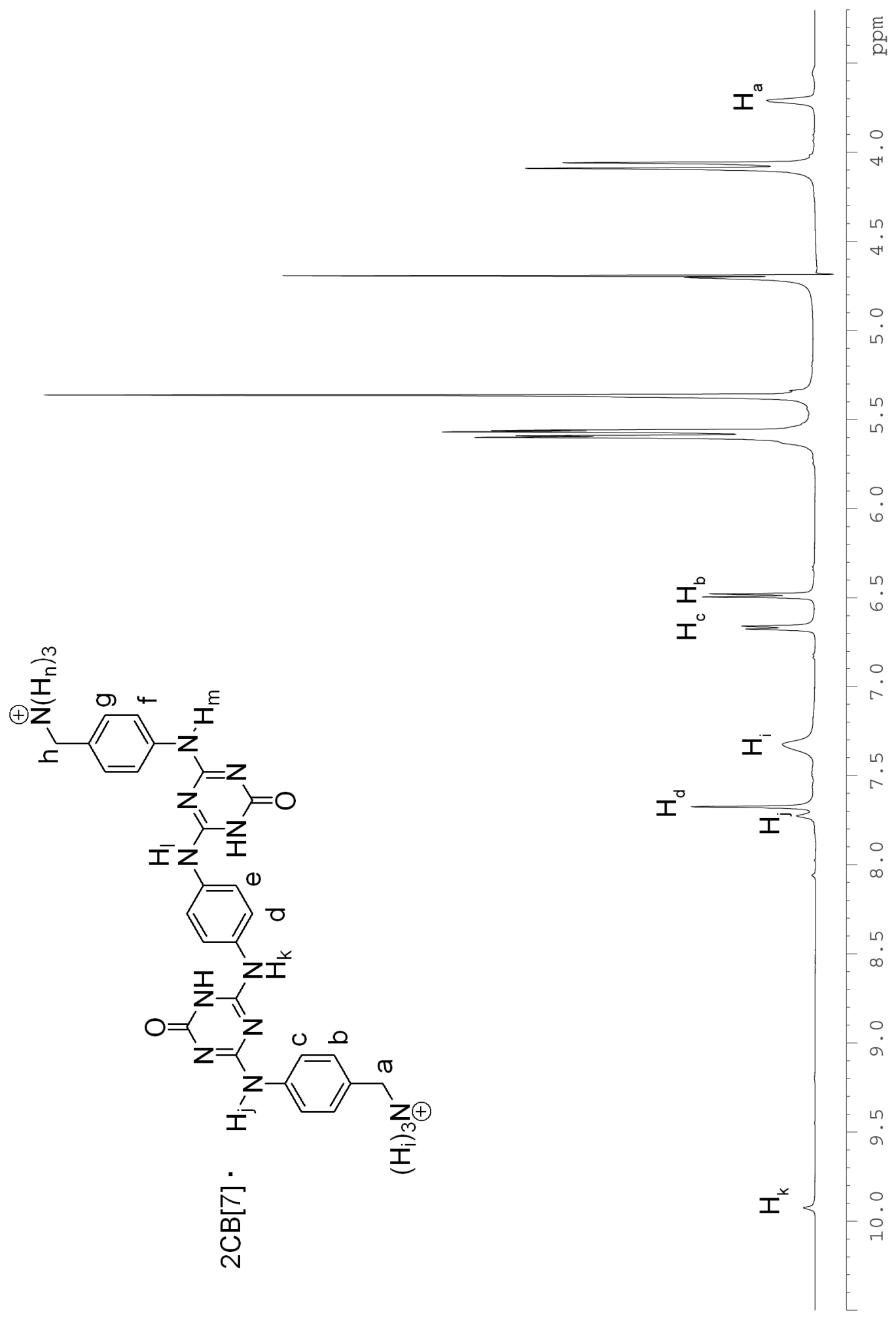

Figure S22. ${ }^{1} \mathrm{H}$ NMR spectrum $\left(500 \mathrm{MHz}, 85 \% \mathrm{H}_{2} \mathrm{O} / \mathrm{D}_{2} \mathrm{O}, \mathrm{RT}\right)$ for $\mathrm{CB}[7] \bullet 3 \bullet \mathrm{CB}[7]$. 


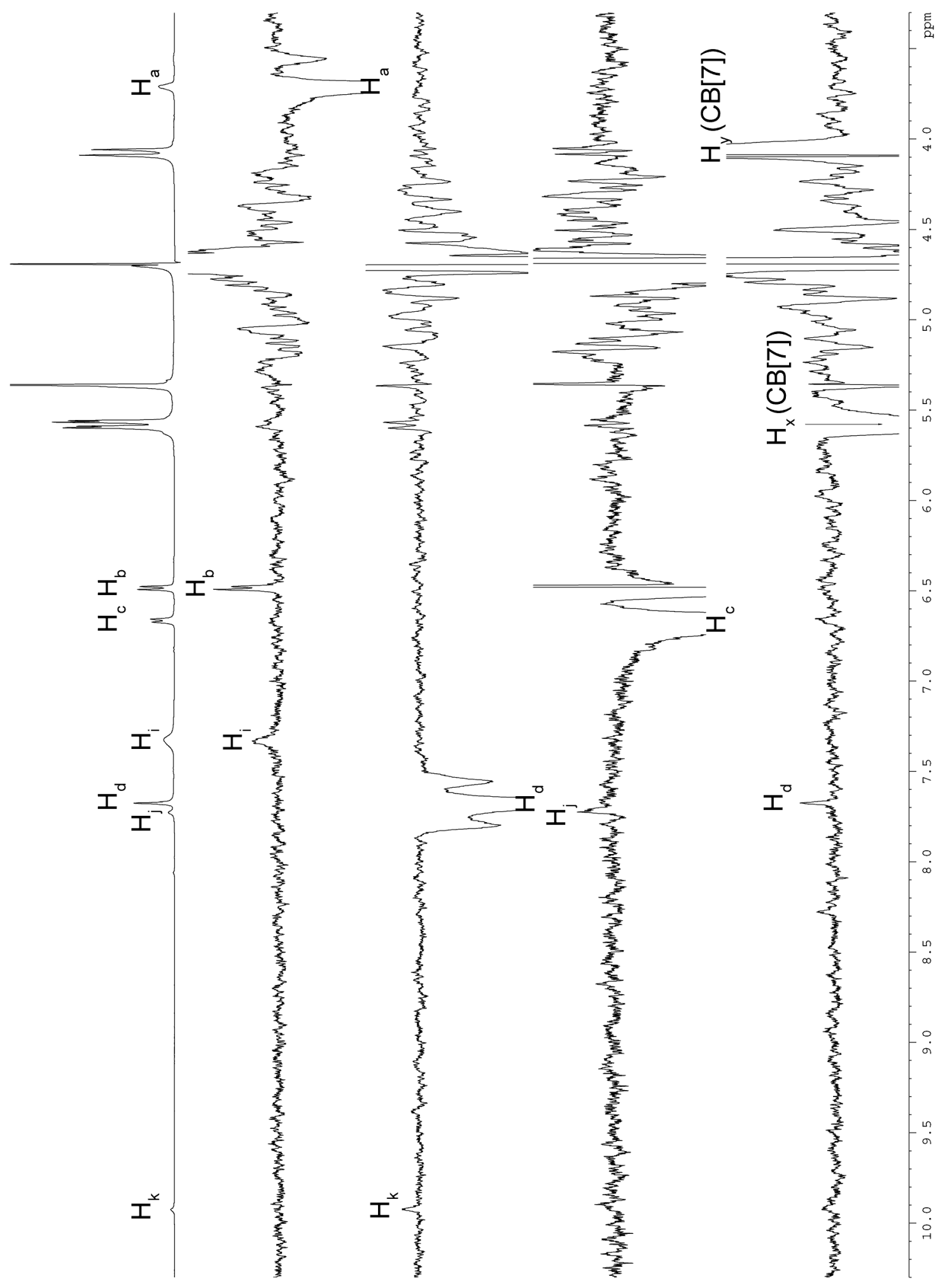

Figure S23. Selective 1D ROE spectra $\left(500 \mathrm{MHz}, 85 \% \mathrm{H}_{2} \mathrm{O} / \mathrm{D}_{2} \mathrm{O}, \mathrm{RT}\right)$ for $\mathrm{CB}[7] \cdot 3 \bullet \mathrm{CB}[7]$. 


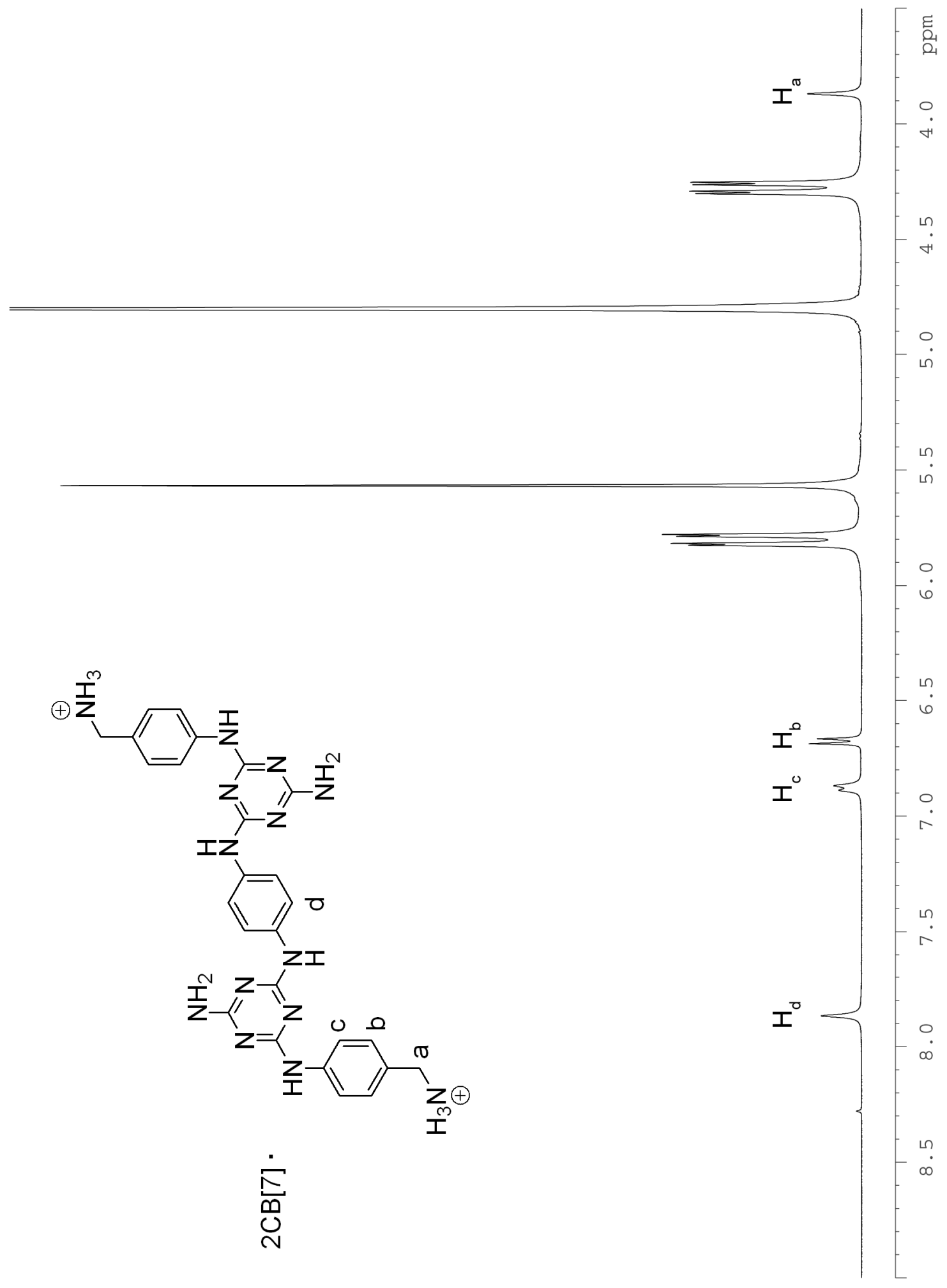

Figure S24. ${ }^{1} \mathrm{H}$ NMR spectrum $\left(400 \mathrm{MHz}, \mathrm{D}_{2} \mathrm{O}, \mathrm{RT}\right)$ for $\mathrm{CB}[7] \bullet 4 \cdot \mathrm{CB}[7]$. 


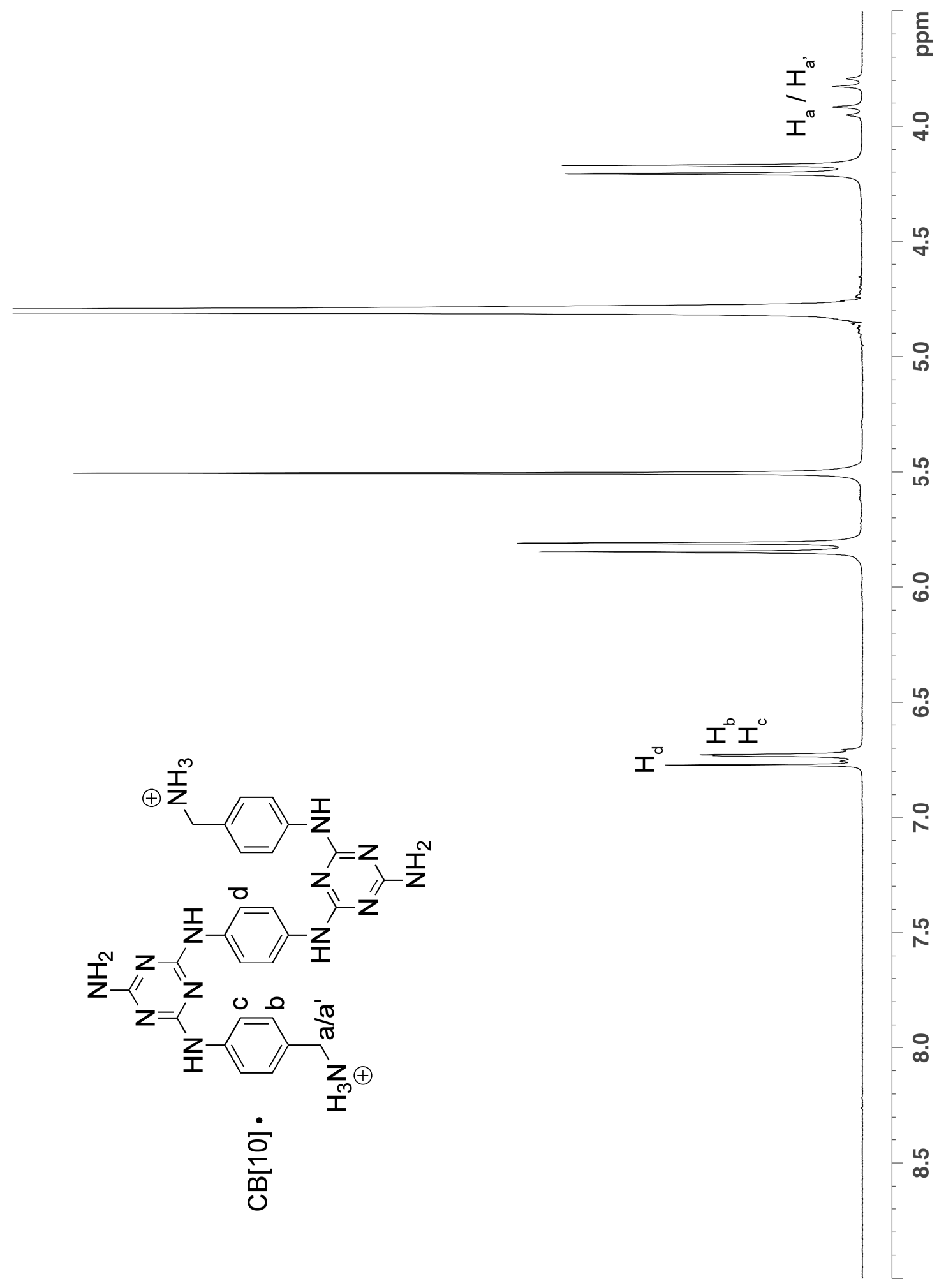

Figure S25. ${ }^{1} \mathrm{H}$ NMR spectrum $\left(400 \mathrm{MHz}, \mathrm{D}_{2} \mathrm{O}, \mathrm{RT}\right)$ for $\mathrm{CB}[10] \bullet 4$. 


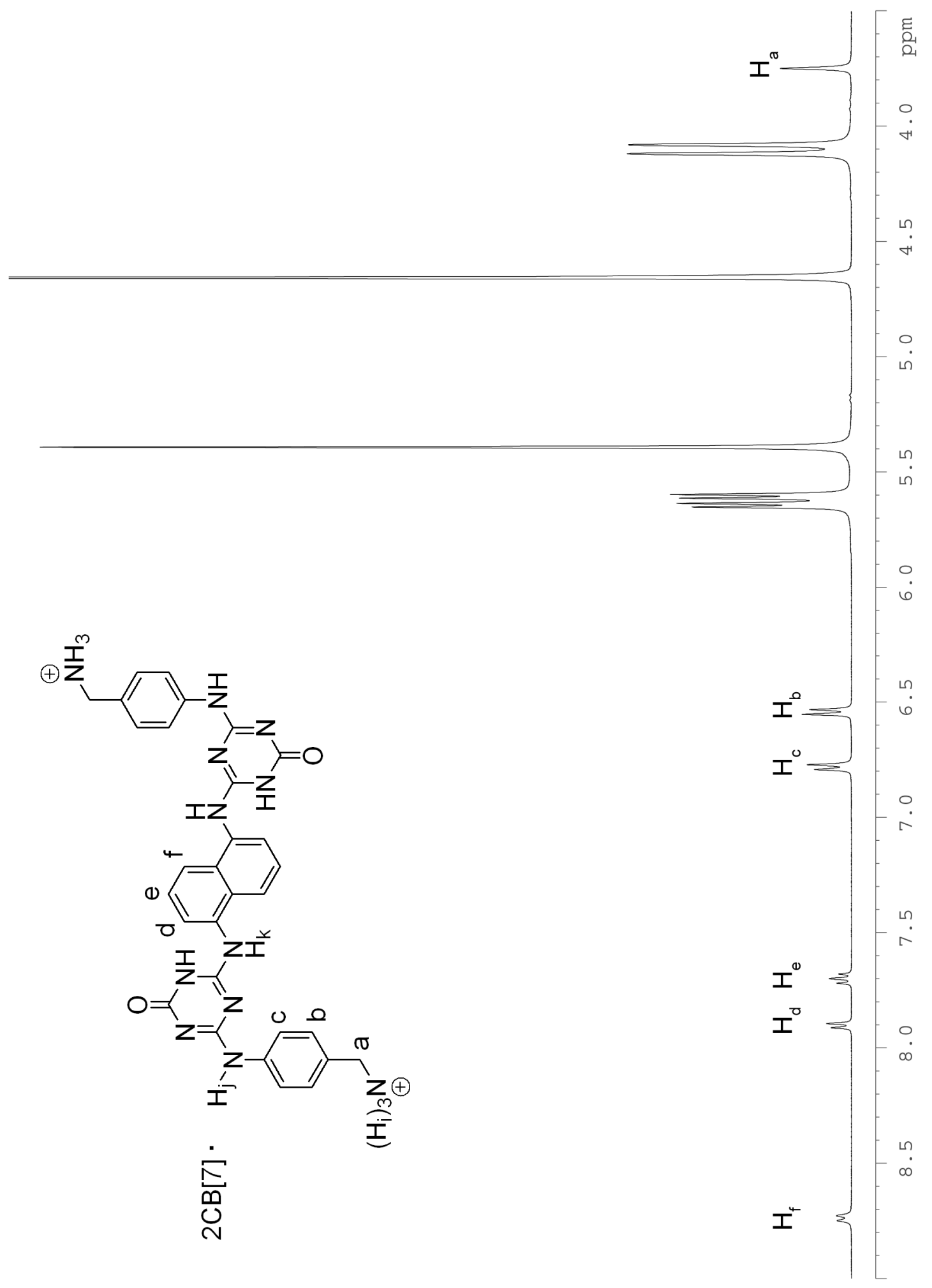

Figure S26. ${ }^{1} \mathrm{H}$ NMR spectrum $\left(400 \mathrm{MHz}, \mathrm{D}_{2} \mathrm{O}, \mathrm{RT}\right)$ for $\mathrm{CB}[7] \bullet 7 \bullet \mathrm{CB}[7]$. 


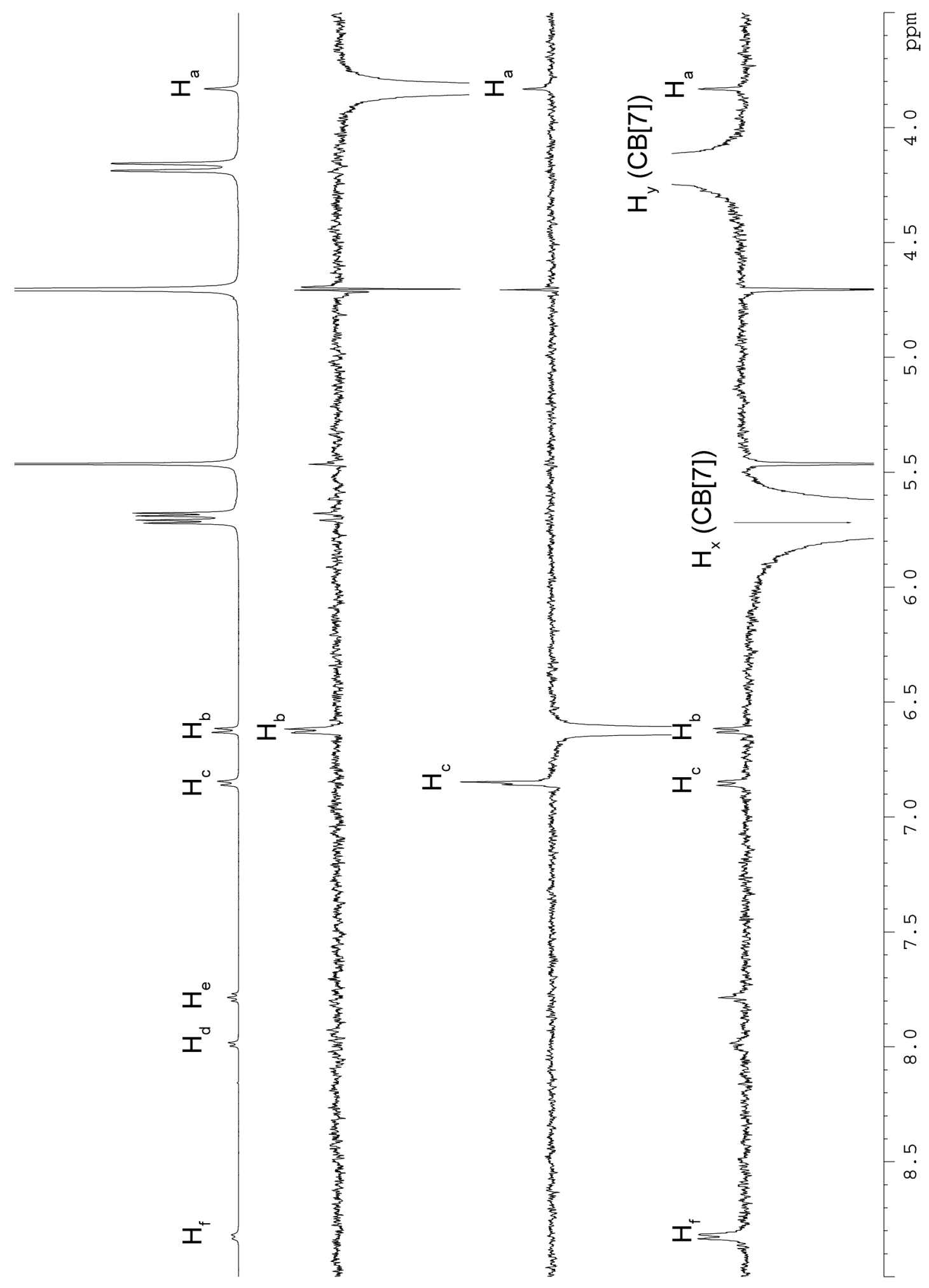

Figure S27. Selective 1D ROE spectra $\left(500 \mathrm{MHz}, \mathrm{D}_{2} \mathrm{O}, \mathrm{RT}\right)$ for $\mathrm{CB}[7] \cdot 7 \cdot \mathrm{CB}[7]$. 


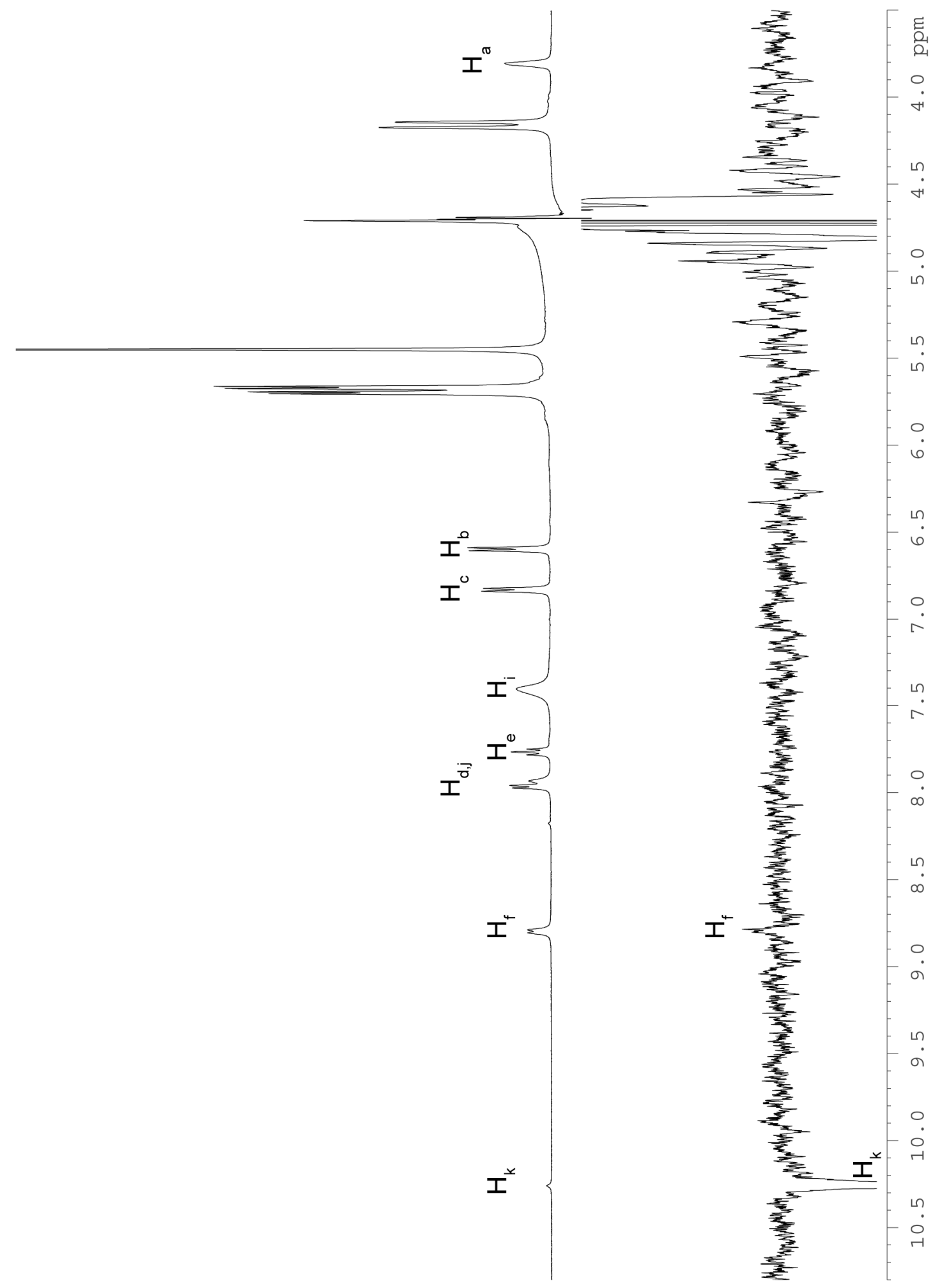

Figure S28. Selective 1D ROE spectra $\left(500 \mathrm{MHz}, 85 \% \mathrm{H}_{2} \mathrm{O} / \mathrm{D}_{2} \mathrm{O}\right.$, RT) for $\mathrm{CB}[7] \cdot 7 \bullet \mathrm{CB}[7]$. 


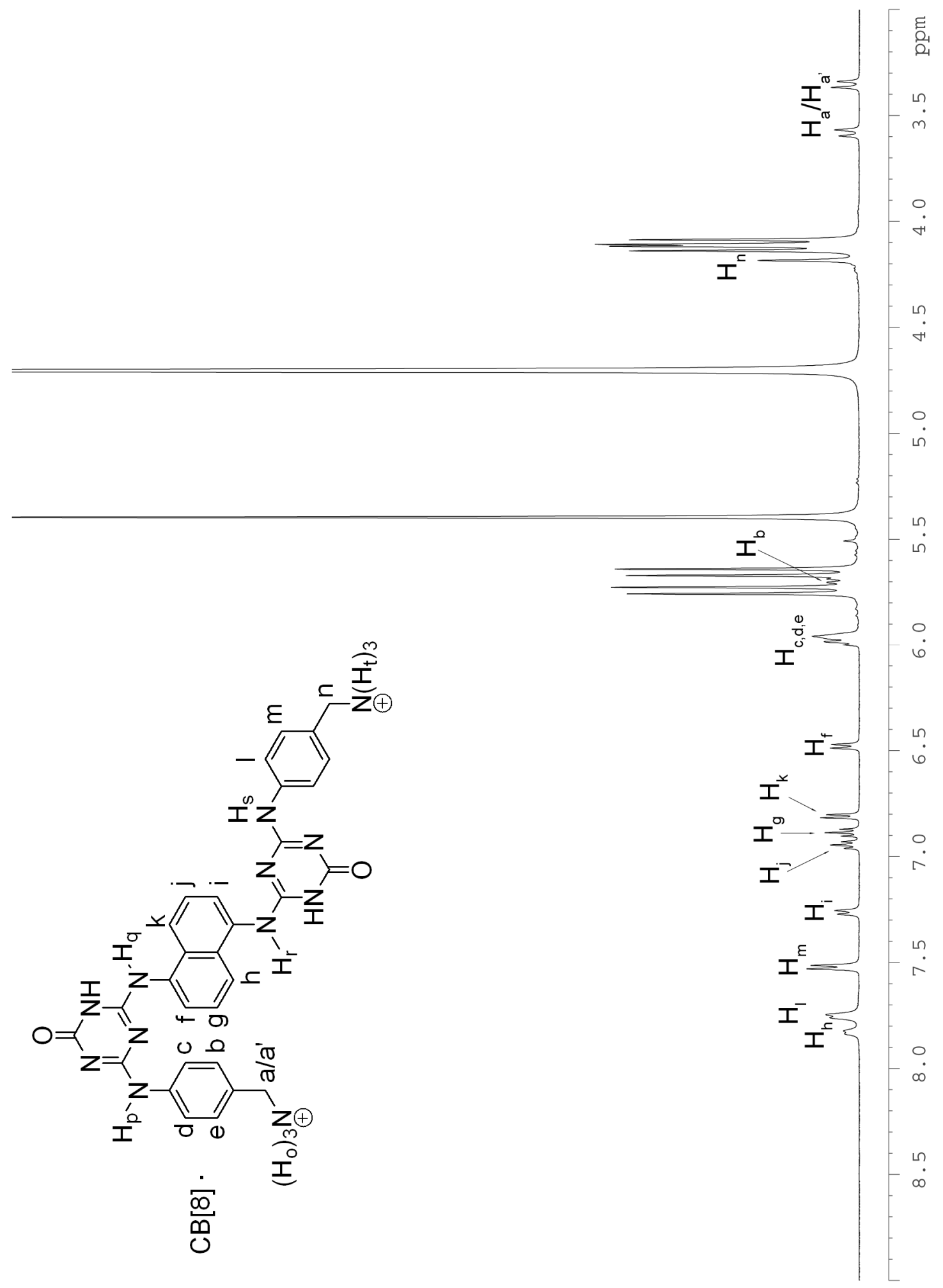

Figure S29. ${ }^{1} \mathrm{H}$ NMR spectrum $\left(500 \mathrm{MHz}, \mathrm{D}_{2} \mathrm{O}\right.$, RT) for $\mathrm{CB}[8] \bullet 7$. 


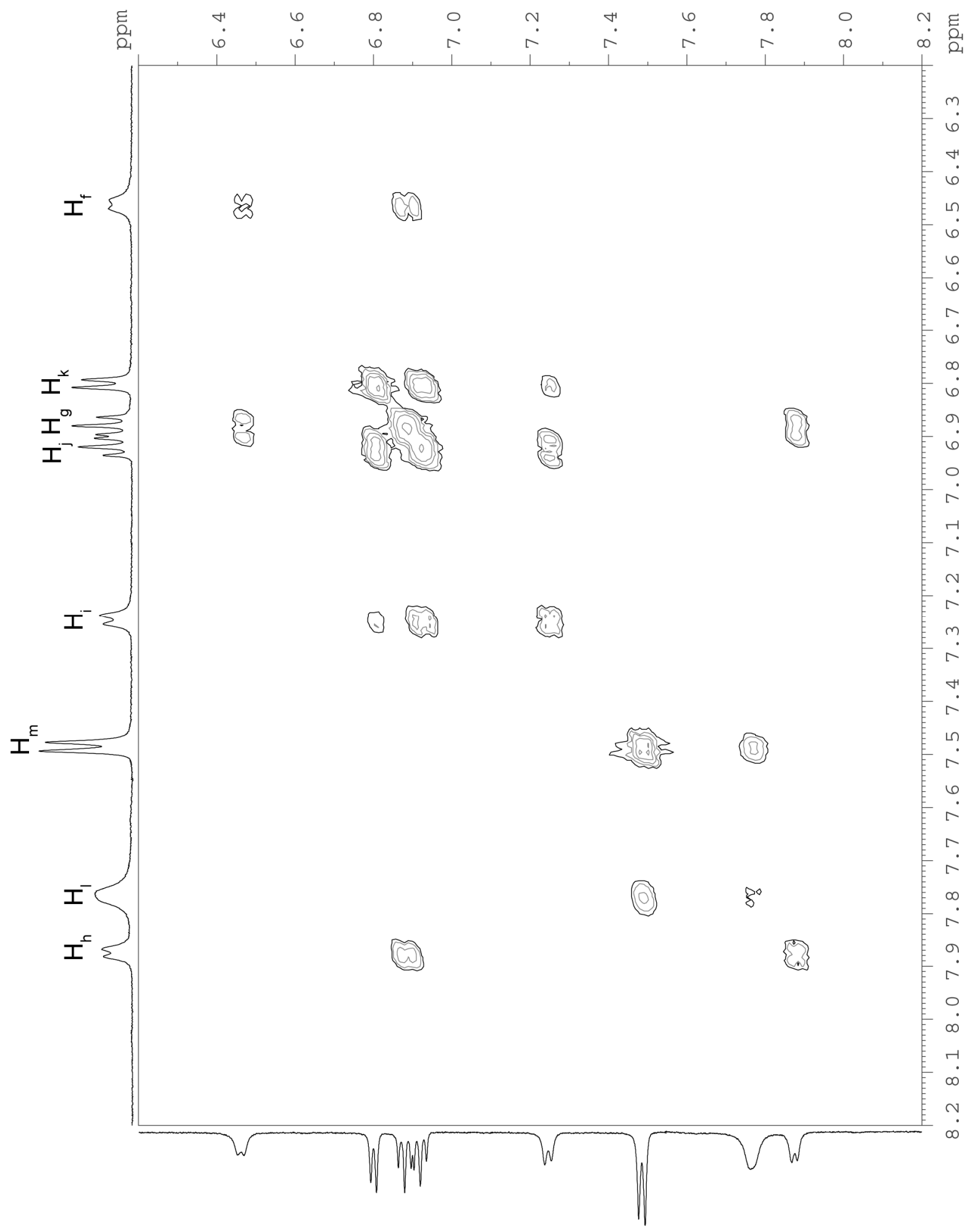

Figure S30. COSY spectrum $\left(500 \mathrm{MHz}, \mathrm{D}_{2} \mathrm{O}, \mathrm{RT}\right)$ for $\mathrm{CB}[8] \bullet 7$. 


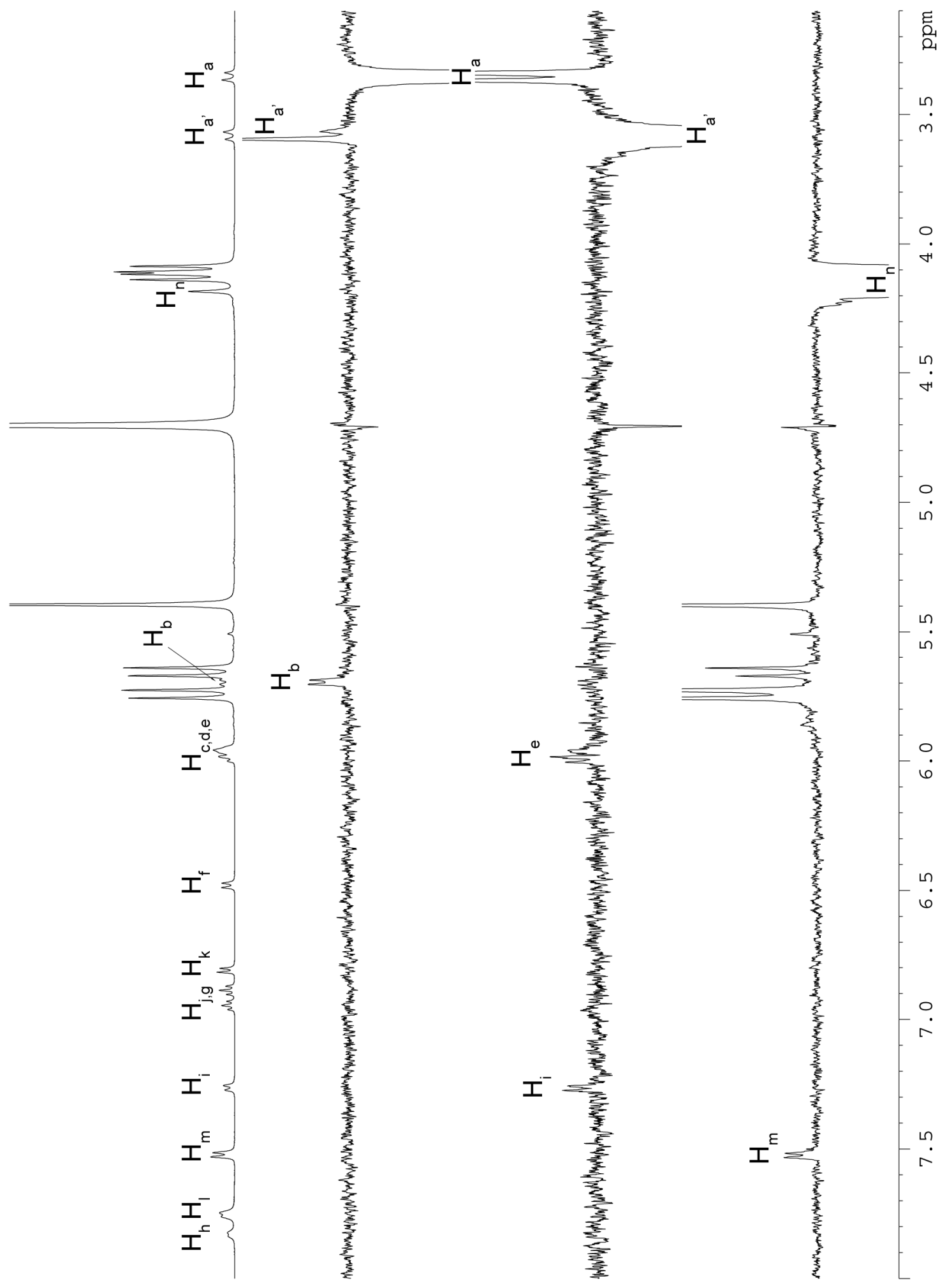

Figure S31. Selective 1D ROE spectra (500 MHz, $\mathrm{D}_{2} \mathrm{O}$, RT) for CB[8]•7. 


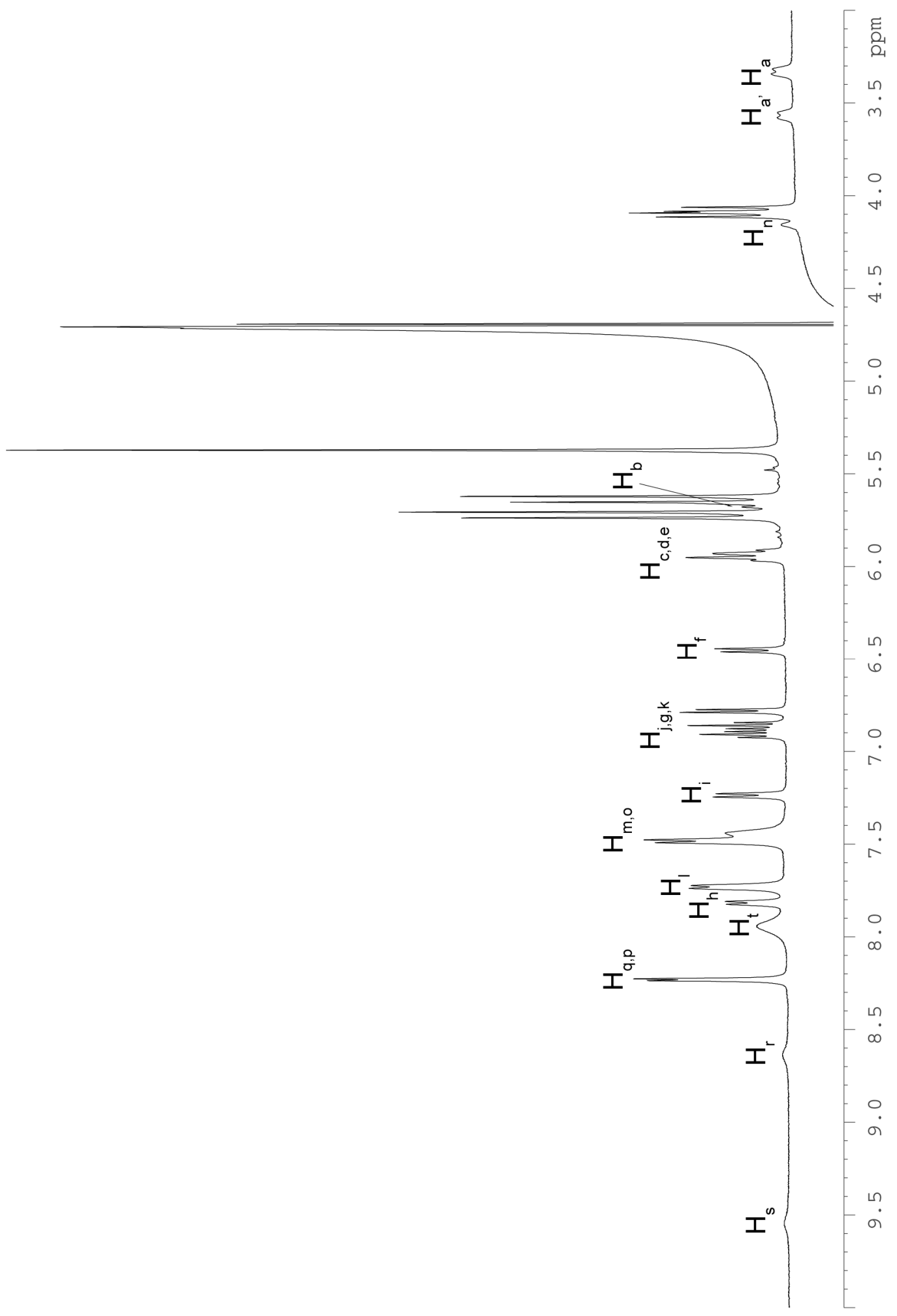

Figure S32. ${ }^{1} \mathrm{H}$ NMR spectrum $\left(500 \mathrm{MHz}, 85 \% \mathrm{H}_{2} \mathrm{O} / \mathrm{D}_{2} \mathrm{O}\right.$, RT) for $\mathrm{CB}[8] \bullet 7$. 


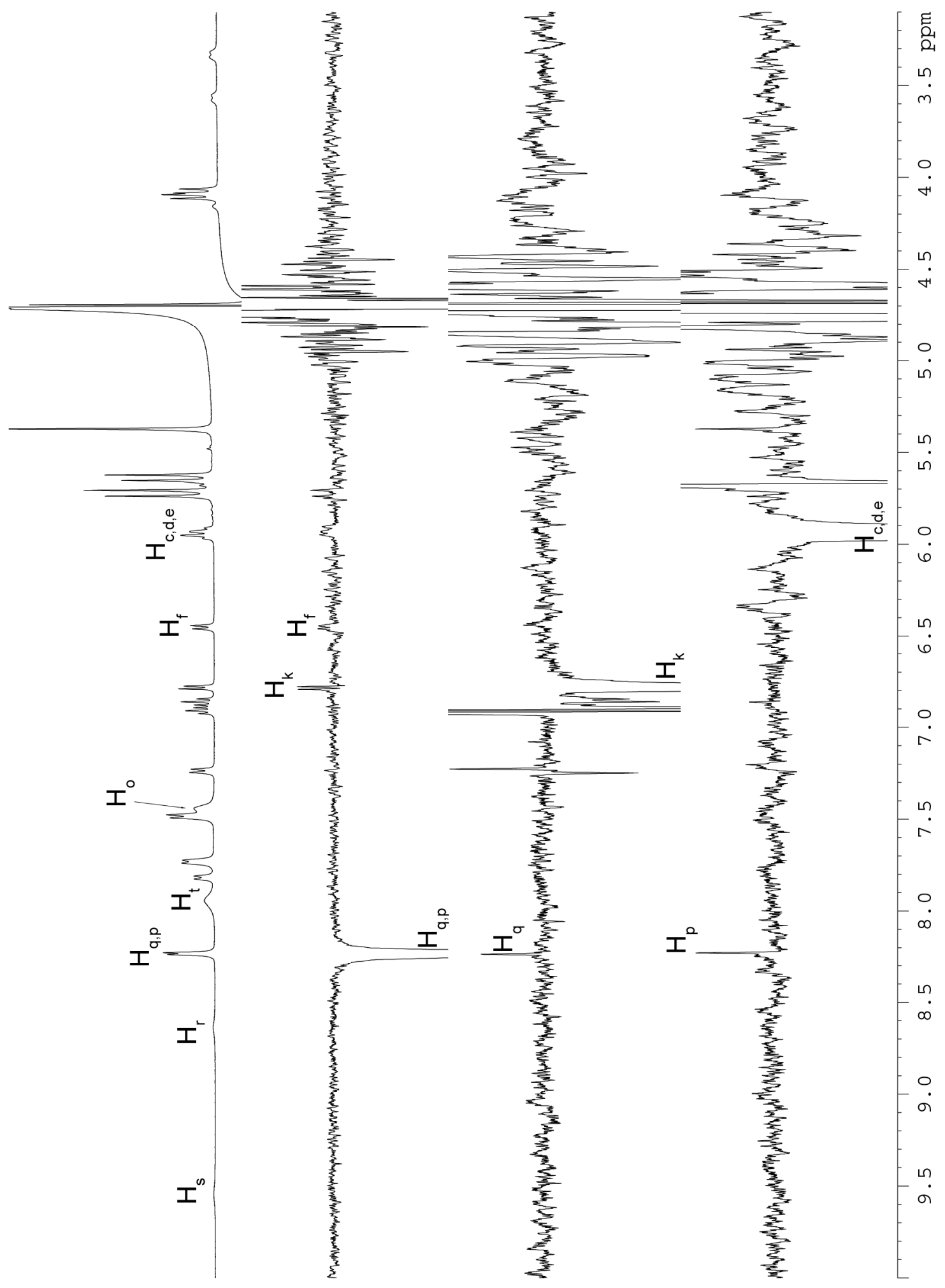

Figure S33. Selective 1D ROE spectra $\left(500 \mathrm{MHz}, 85 \% \mathrm{H}_{2} \mathrm{O} / \mathrm{D}_{2} \mathrm{O}, \mathrm{RT}\right)$ for $\mathrm{CB}[8] \bullet 7$. 


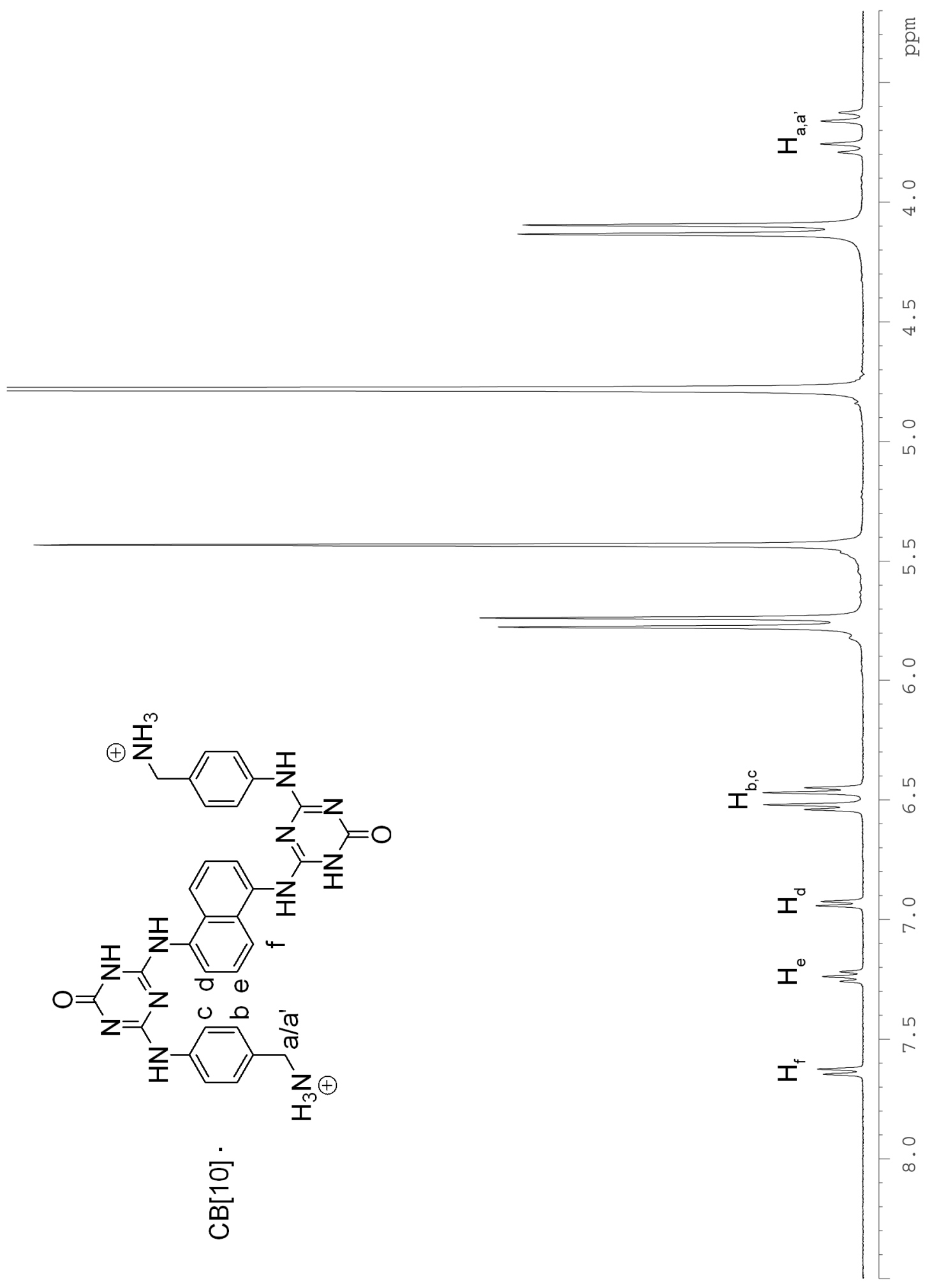

Figure S34. 1H NMR spectrum (400 MHz, $\mathrm{D}_{2} \mathrm{O}$, RT) for $\mathrm{CB}[10] \bullet 7$. 


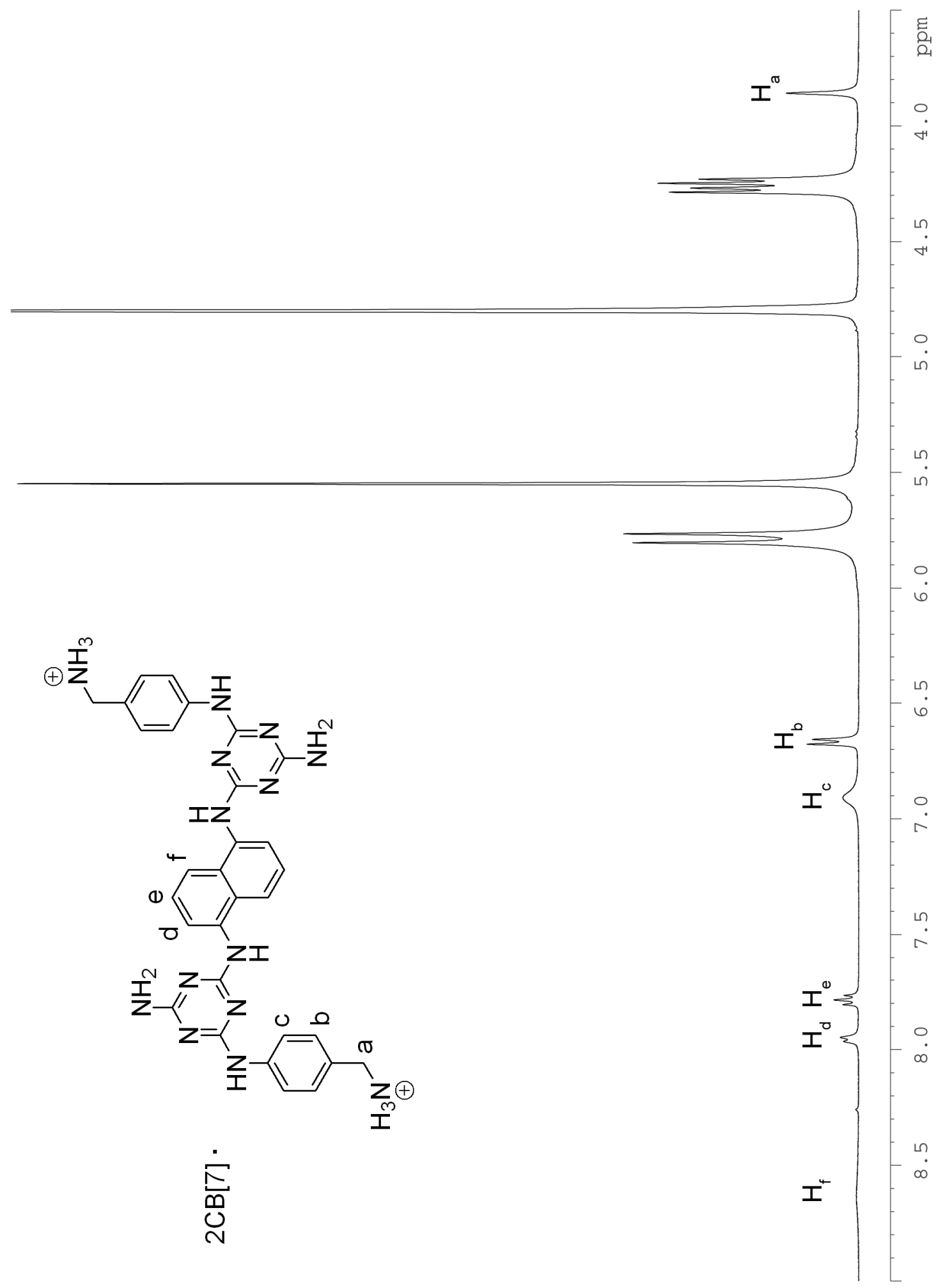

Figure S35. ${ }^{1} \mathrm{H}$ NMR spectrum (400 $\left.\mathrm{MHz}, \mathrm{D}_{2} \mathrm{O}, \mathrm{RT}\right)$ for $\mathrm{CB}[7] \bullet 8 \bullet \mathrm{CB}[7]$. 


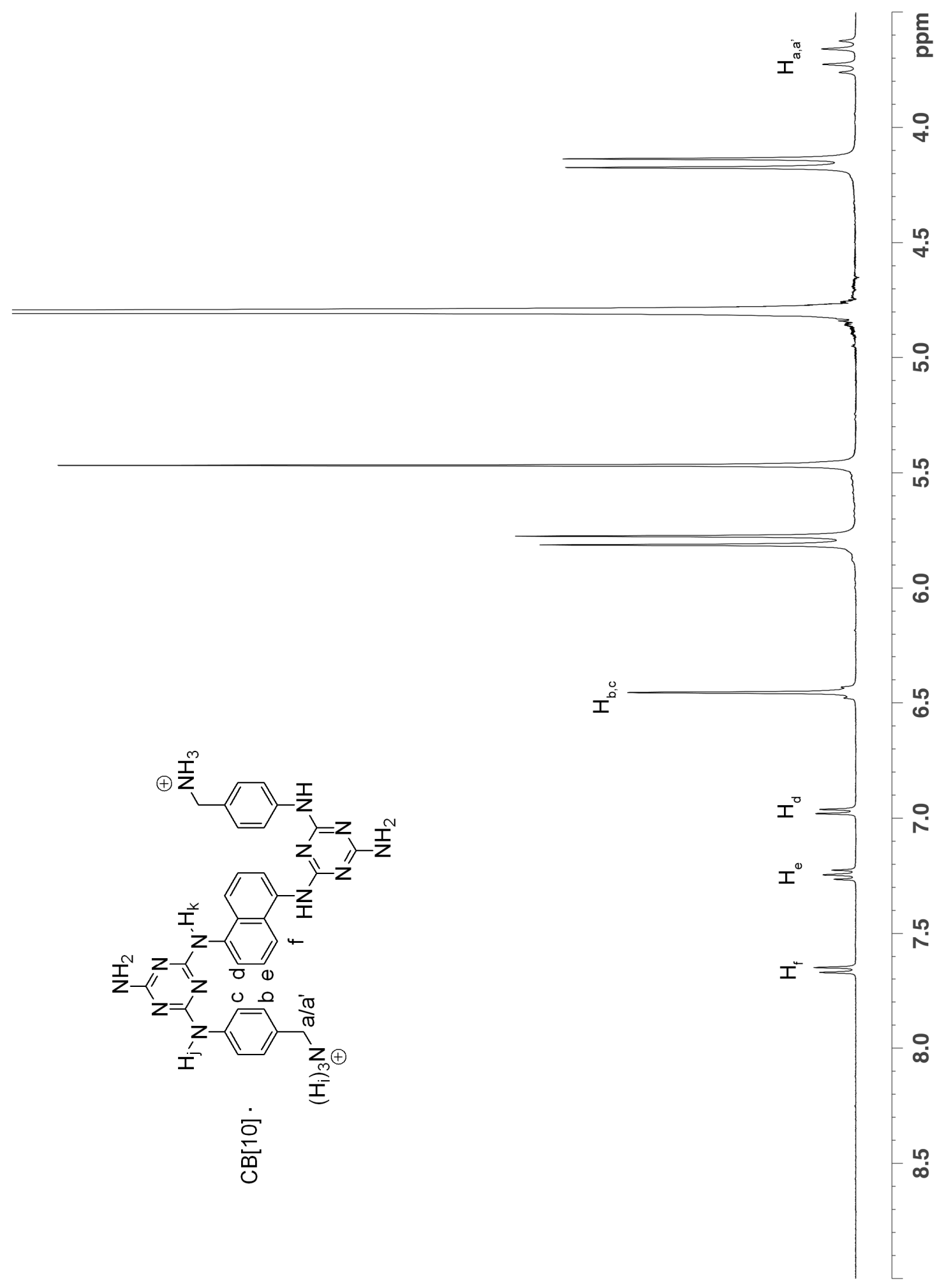

Figure S36. ${ }^{1} \mathrm{H}$ NMR spectrum $\left(400 \mathrm{MHz}, \mathrm{D}_{2} \mathrm{O}, \mathrm{RT}\right)$ for $\mathrm{CB}[10] \bullet 8$. 


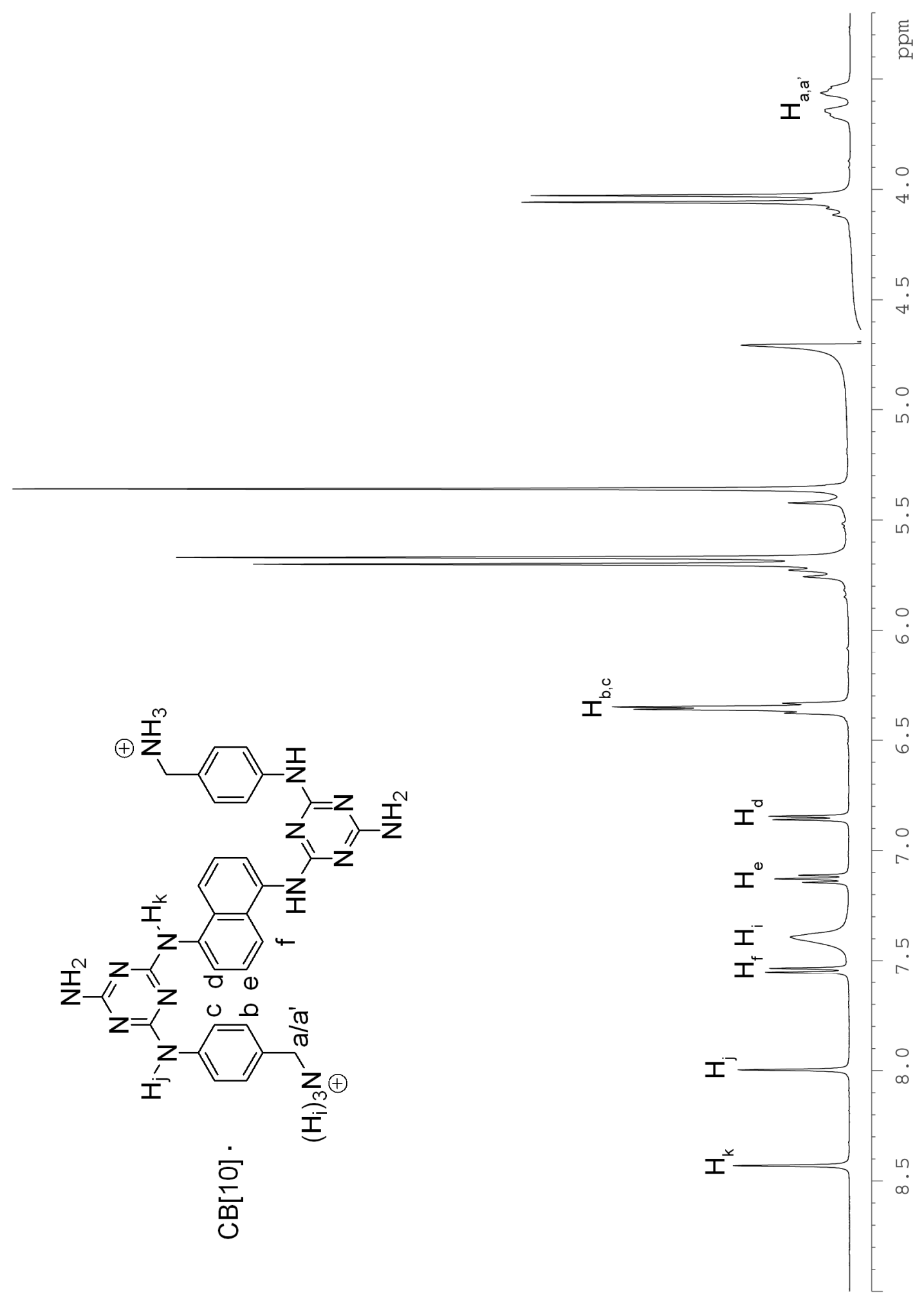

Figure S37. ${ }^{1} \mathrm{H}$ NMR spectrum $\left(500 \mathrm{MHz}, 85 \% \mathrm{H}_{2} \mathrm{O} / \mathrm{D}_{2} \mathrm{O}, \mathrm{RT}\right)$ for $\mathrm{CB}[10] \bullet 8$. 


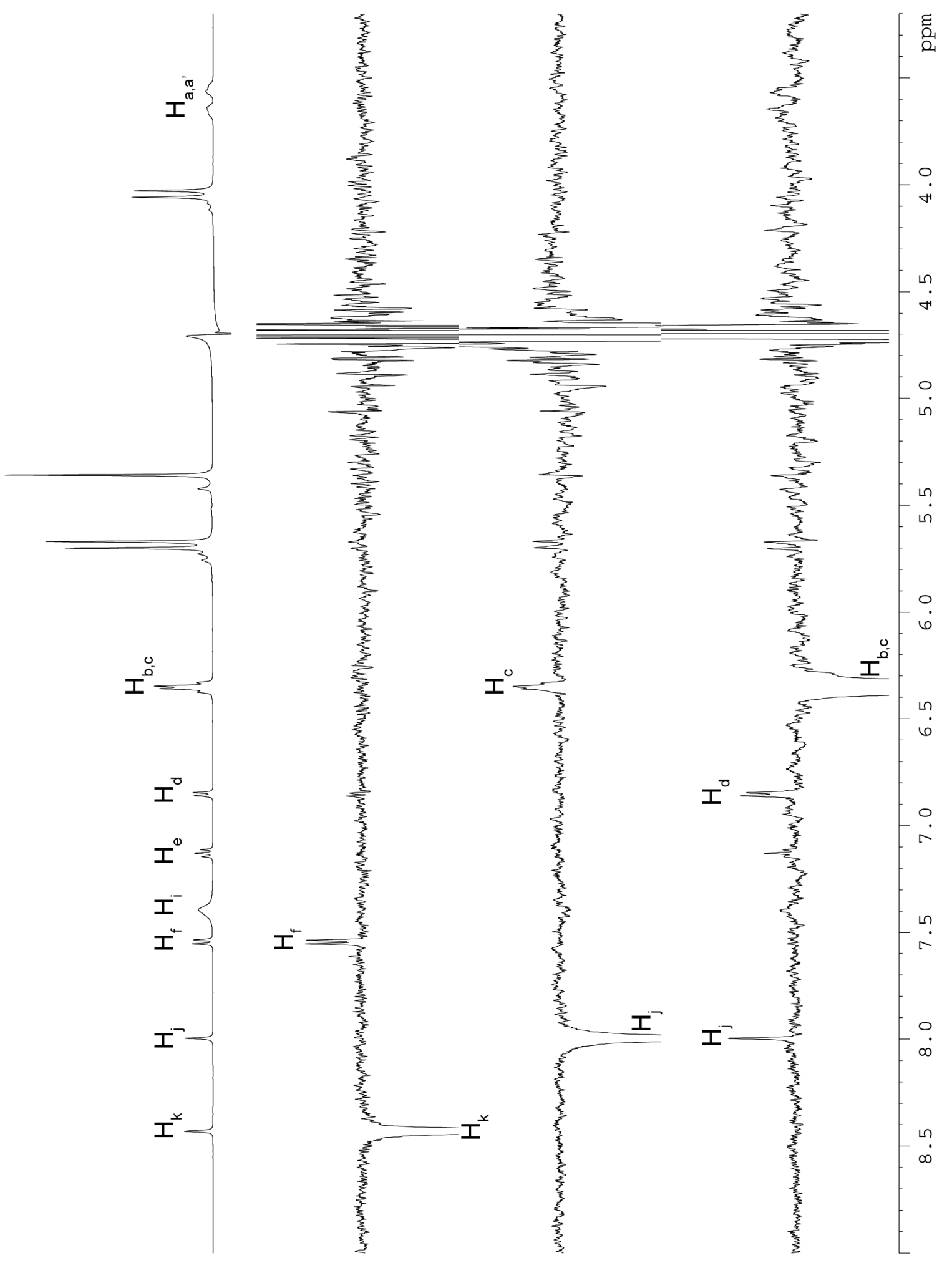

Figure S38. Selective 1D ROE spectra (500 MHz, $85 \% \mathrm{H}_{2} \mathrm{O} / \mathrm{D}_{2} \mathrm{O}$, RT) for CB[10]•8. 


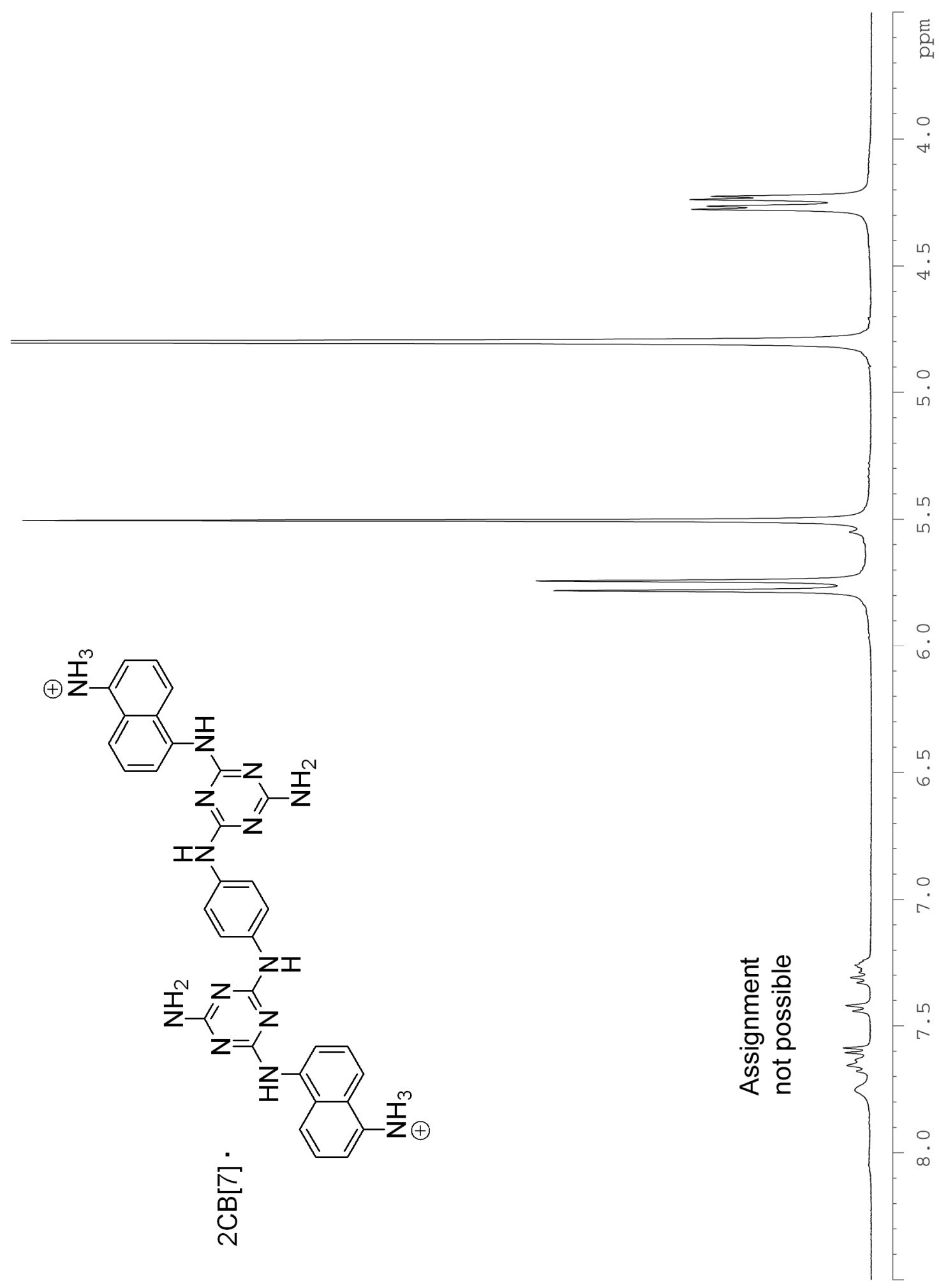

Figure S39. ${ }^{1} \mathrm{H} \mathrm{NMR}$ spectrum $\left(400 \mathrm{MHz}, \mathrm{D}_{2} \mathrm{O}, \mathrm{RT}\right)$ for $\mathrm{CB}[7] \bullet 11 \bullet \mathrm{CB}[7]$. 


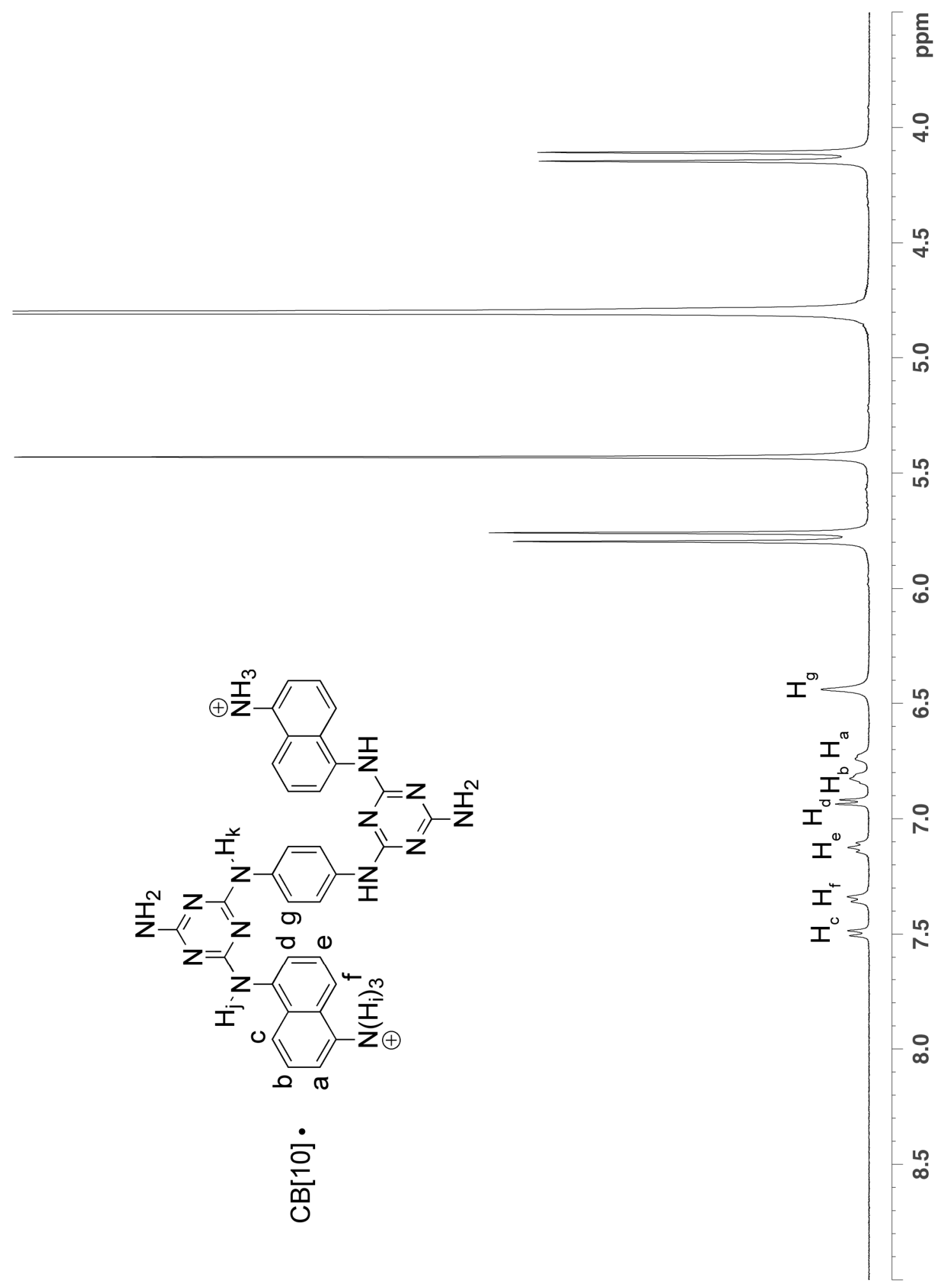

Figure S40. ${ }^{1} \mathrm{H}$ NMR spectrum $\left(400 \mathrm{MHz}, \mathrm{D}_{2} \mathrm{O}, \mathrm{RT}\right)$ for $\mathrm{CB}[10] \bullet 11$. 


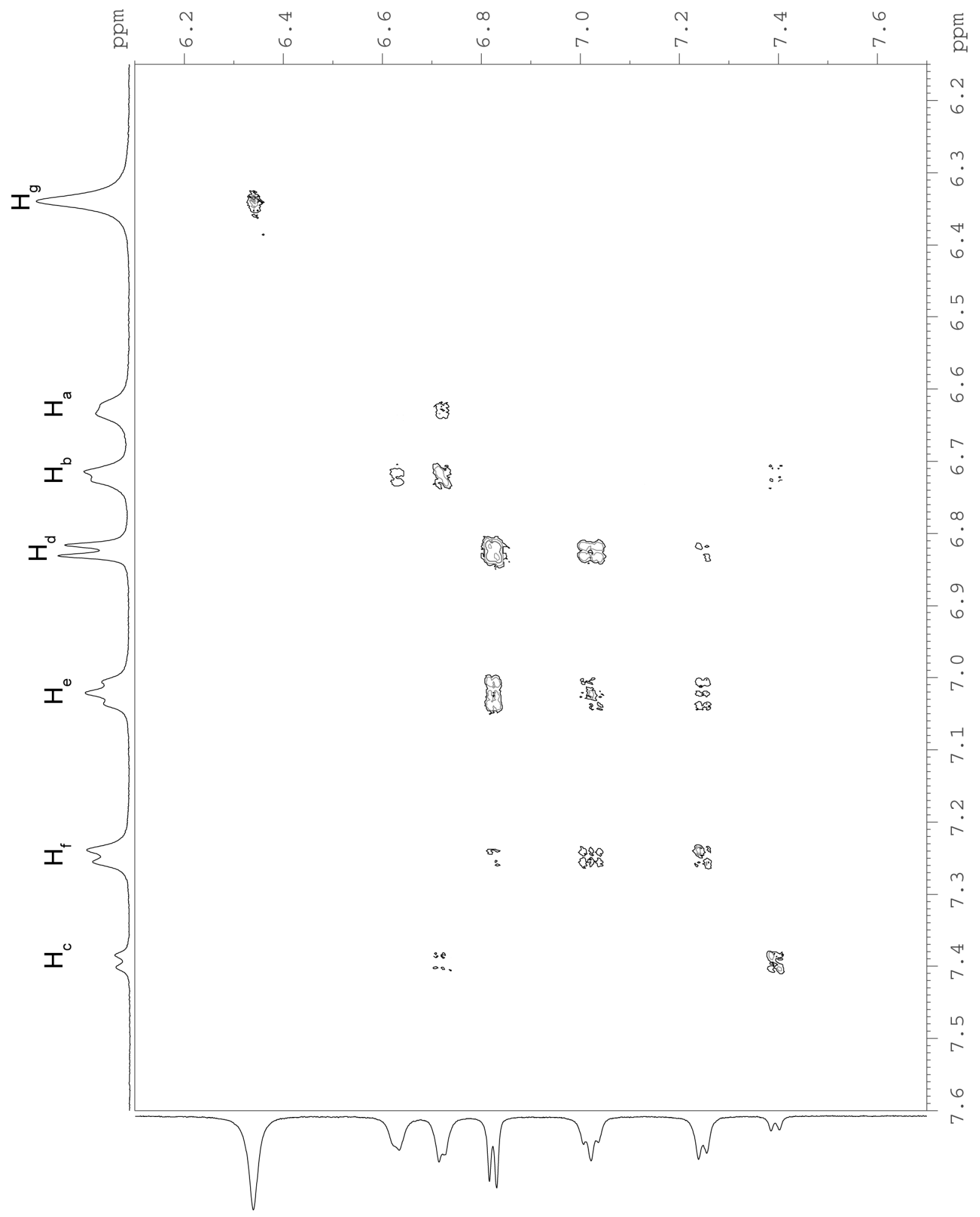

Figure S41. COSY spectrum (500 MHz, $\left.\mathrm{D}_{2} \mathrm{O}, \mathrm{RT}\right)$ for $\mathrm{CB}[10] \bullet 11$. 


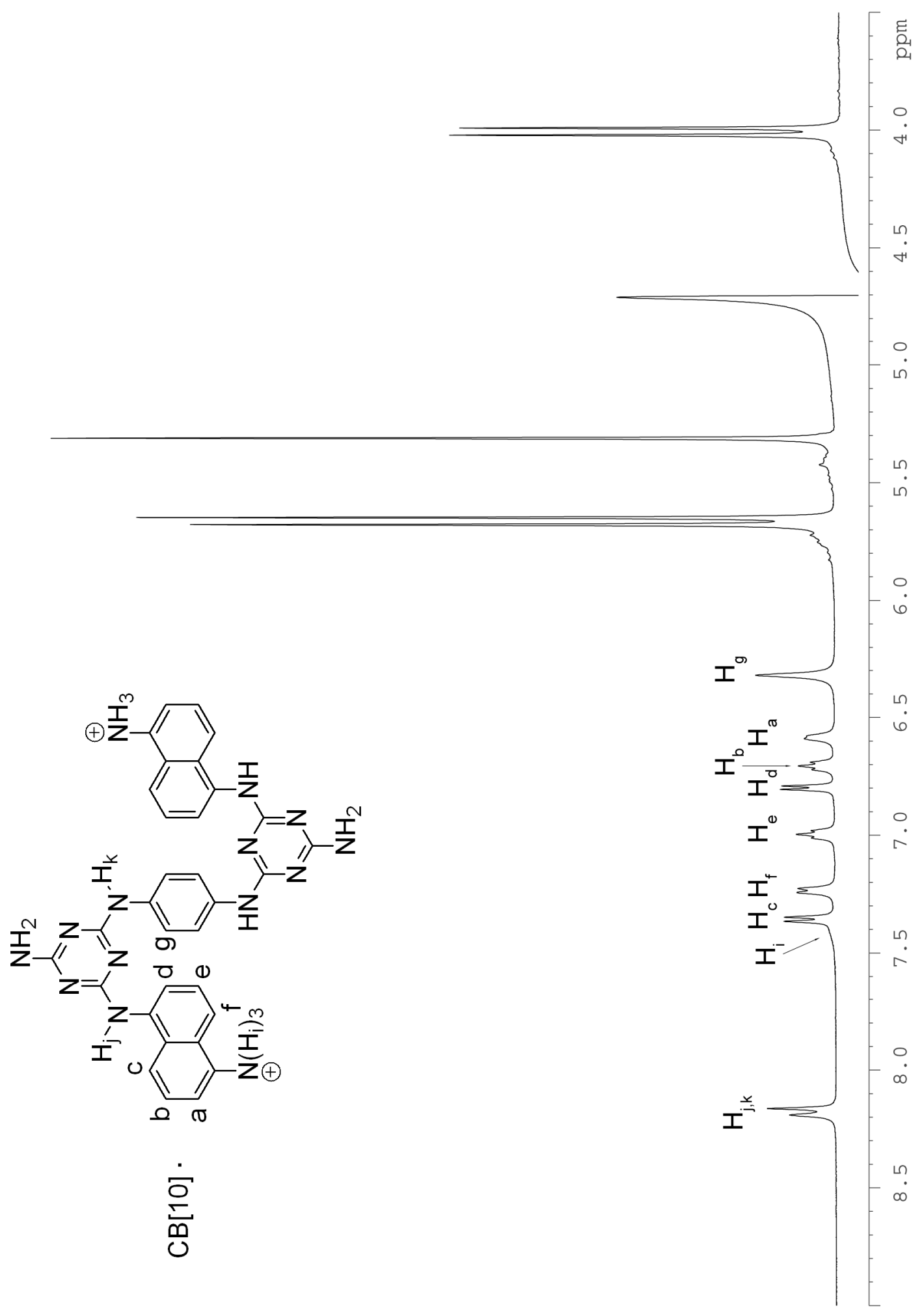

Figure S42. ${ }^{1} \mathrm{H}$ NMR spectrum $\left(500 \mathrm{MHz}, 85 \% \mathrm{H}_{2} \mathrm{O} / \mathrm{D}_{2} \mathrm{O}, \mathrm{RT}\right)$ for $\mathrm{CB}[10] \bullet 11$. 

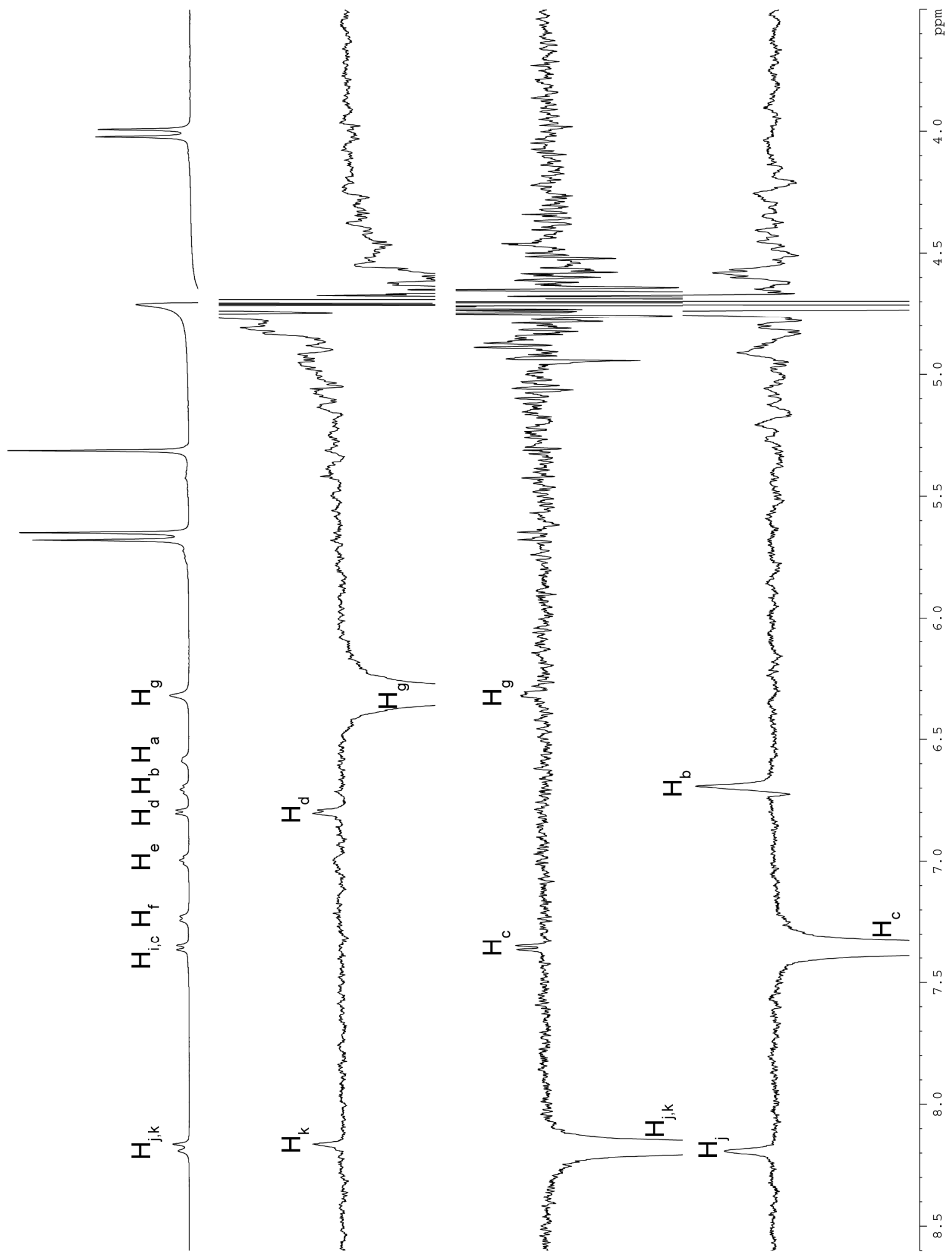

Figure S43. Selective 1D ROE spectra $\left(500 \mathrm{MHz}, 85 \% \mathrm{H}_{2} \mathrm{O} / \mathrm{D}_{2} \mathrm{O}, \mathrm{RT}\right)$ for $\mathrm{CB}[10] \bullet 11$. 


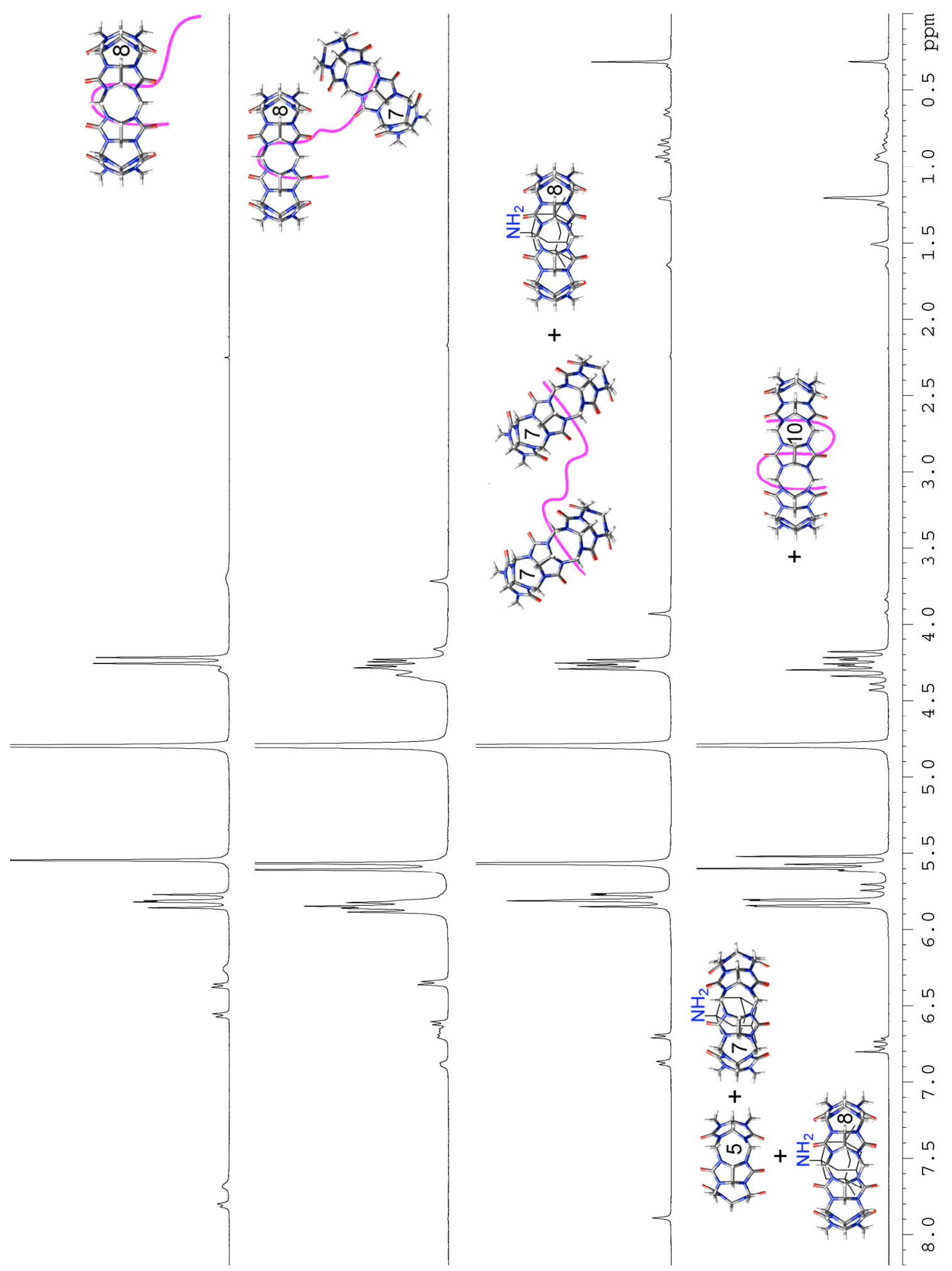

Figure S44. ${ }^{1} \mathrm{H}$ NMR spectra (400 MHz, $\left.\mathrm{D}_{2} \mathrm{O}, \mathrm{RT}\right)$ for the refolding of oligomer 3 in response to the sequential addition of $\mathrm{CB}[8], \mathrm{CB}[7], \mathbf{1 7}$, and $\mathrm{CB}[10] \cdot \mathrm{CB}[5]$ with $\mathbf{1 8}$. 


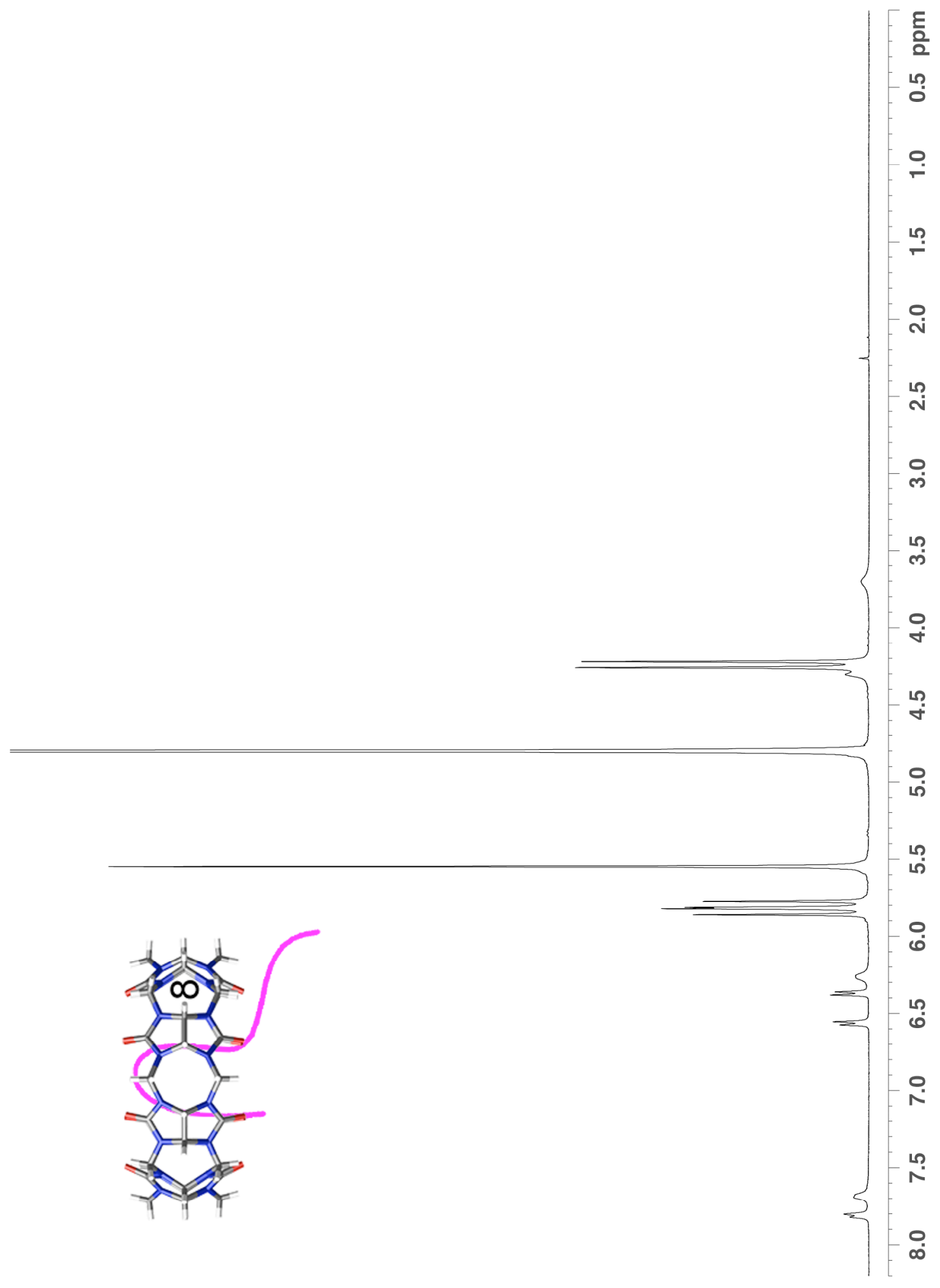

Figure S45. ${ }^{1} \mathrm{H}$ NMR spectra $\left(400 \mathrm{MHz}, \mathrm{D}_{2} \mathrm{O}, \mathrm{RT}\right)$ for $\mathrm{CB}[8] \bullet 3$. 


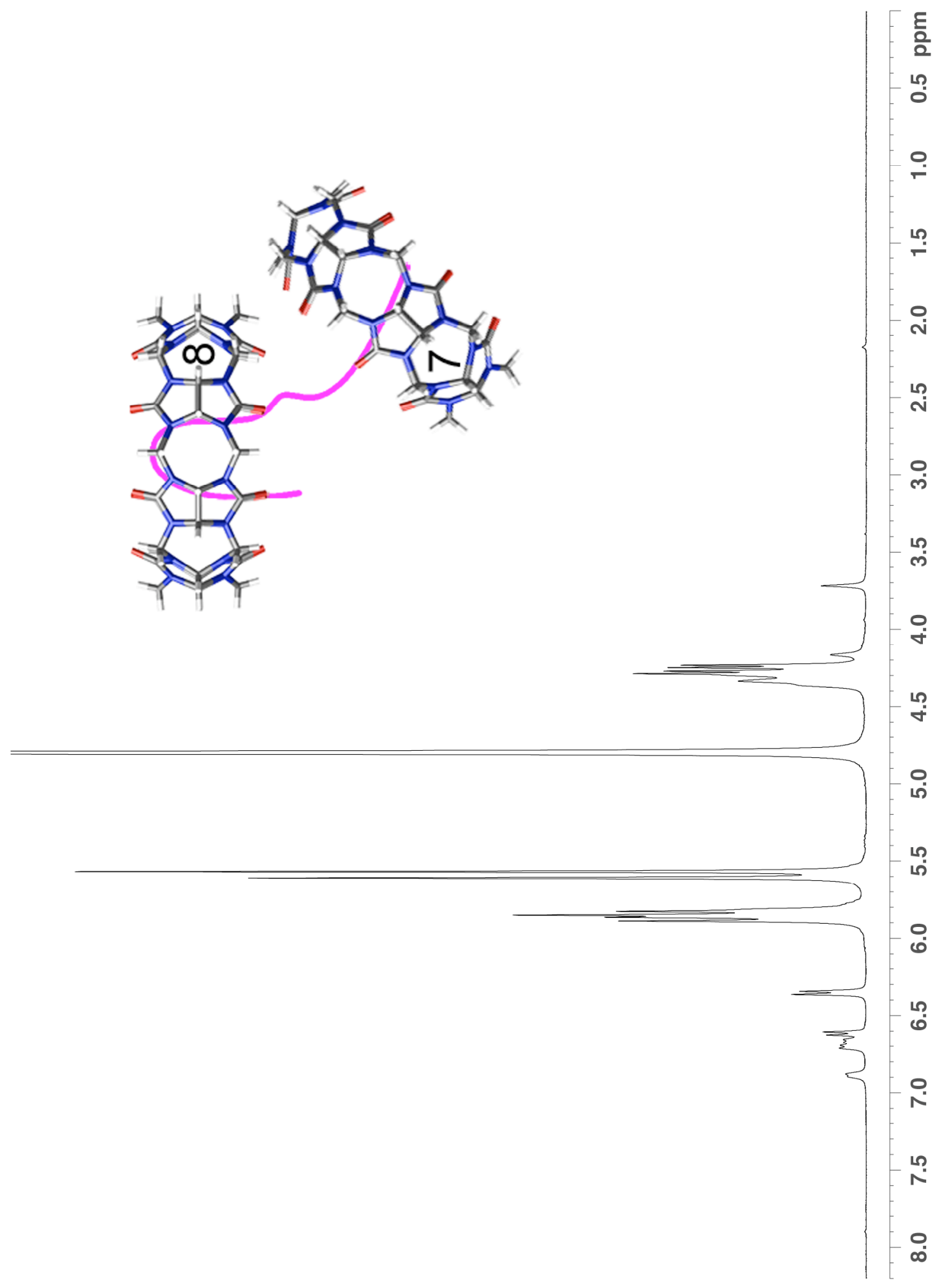

Figure S46. ${ }^{1} \mathrm{H} \mathrm{NMR}$ spectra $\left(400 \mathrm{MHz}, \mathrm{D}_{2} \mathrm{O}, \mathrm{RT}\right)$ for $\mathrm{CB}[8] \bullet 3 \bullet \mathrm{CB}[7]$. 


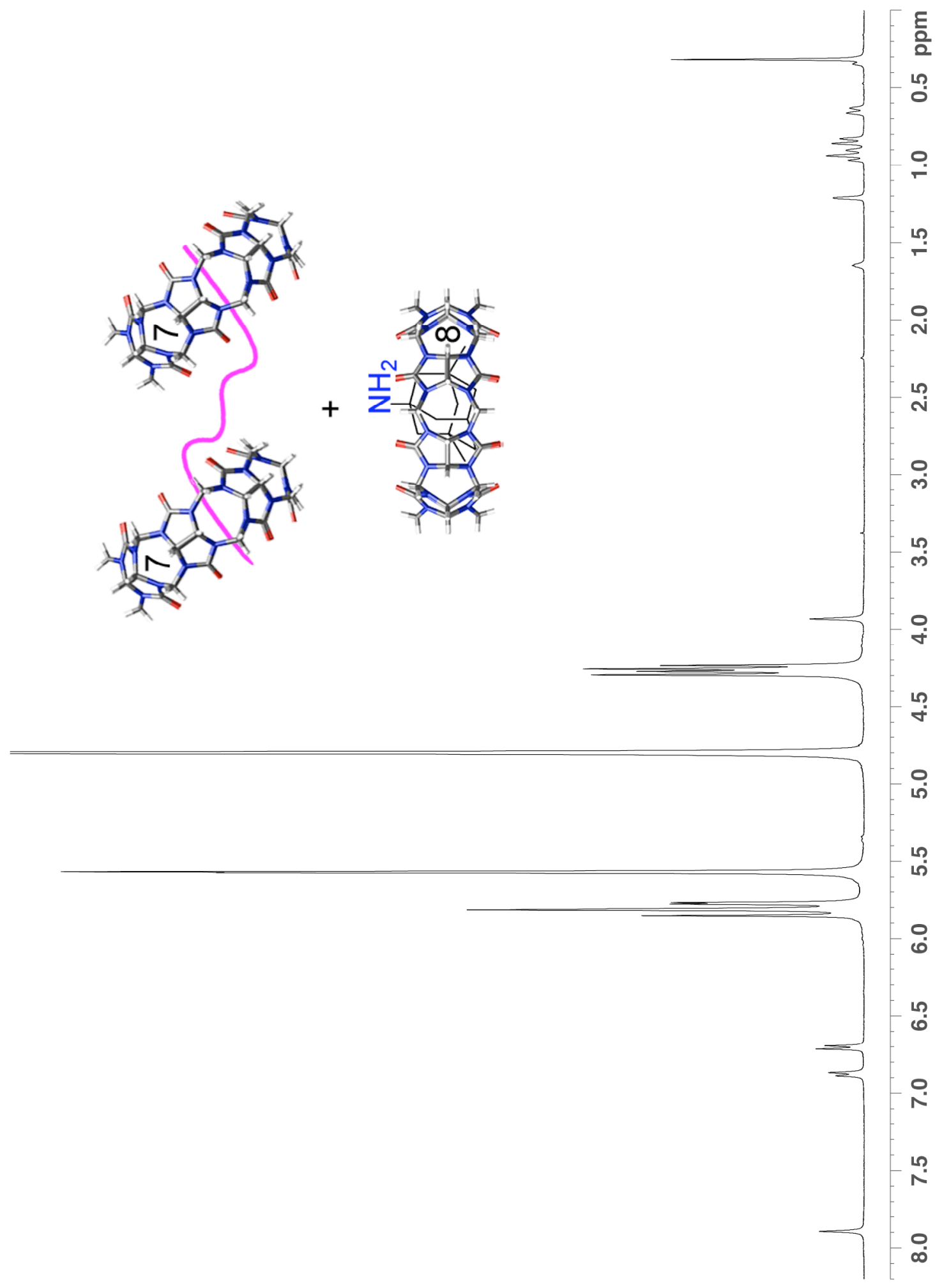

Figure S47. ${ }^{1} \mathrm{H} \mathrm{NMR}$ spectra $\left(400 \mathrm{MHz}, \mathrm{D}_{2} \mathrm{O}, \mathrm{RT}\right)$ for a mixture of $\mathrm{CB}[8] \bullet \mathbf{1 7}+\mathrm{CB}[7] \bullet \mathbf{3} \bullet \mathrm{CB}[7]$ prepared by sequential addition. 


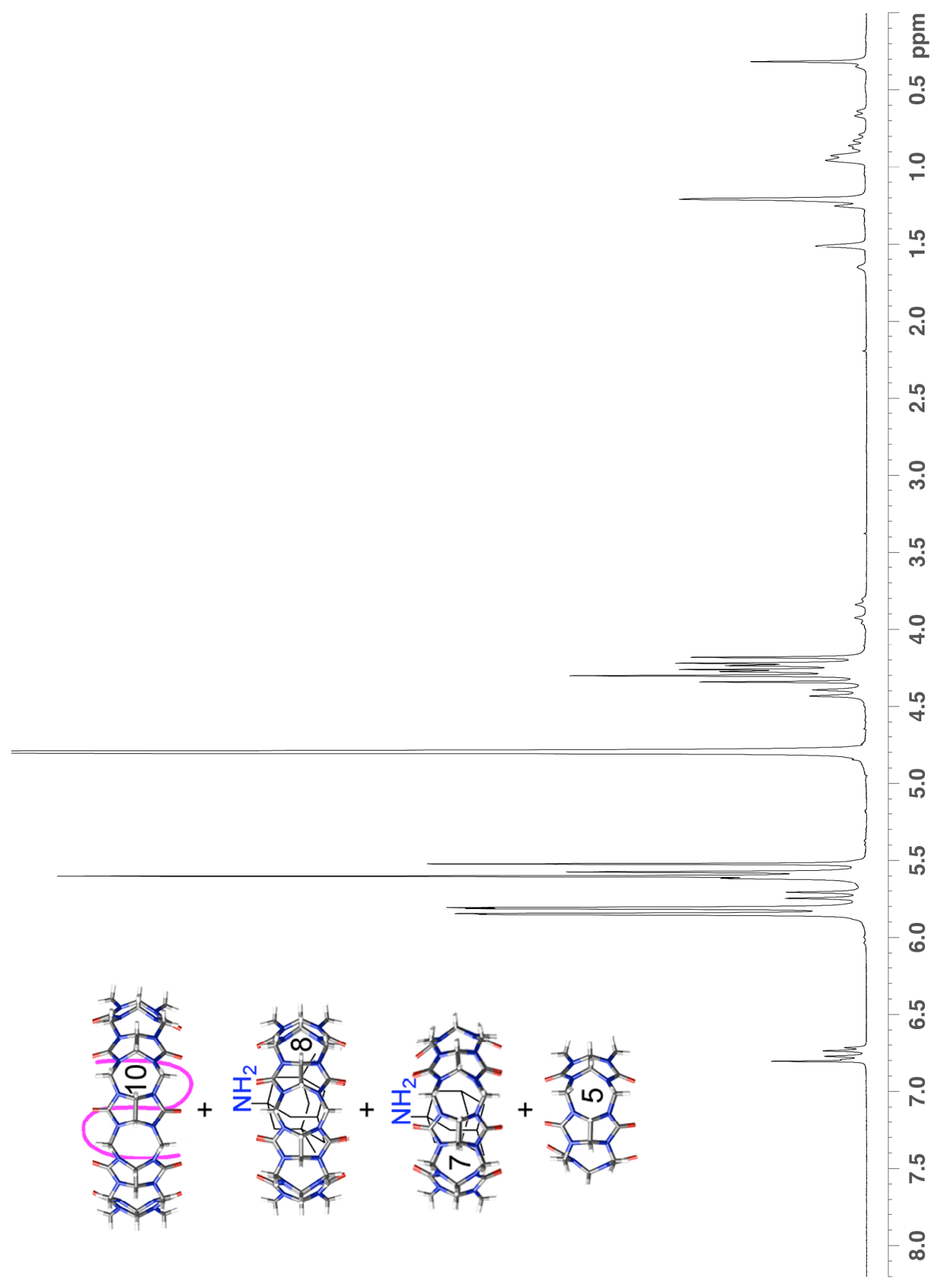

Figure S48. ${ }^{1} \mathrm{H}$ NMR spectra $\left(400 \mathrm{MHz}, \mathrm{D}_{2} \mathrm{O}, \mathrm{RT}\right)$ for a mixture of $\mathrm{CB}[10] \bullet \mathbf{3}+\mathrm{CB}[8] \bullet \mathbf{1 7}+2$ $\mathrm{CB}[7] \bullet 18+\mathrm{CB}[5]$ prepared by sequential addition. 


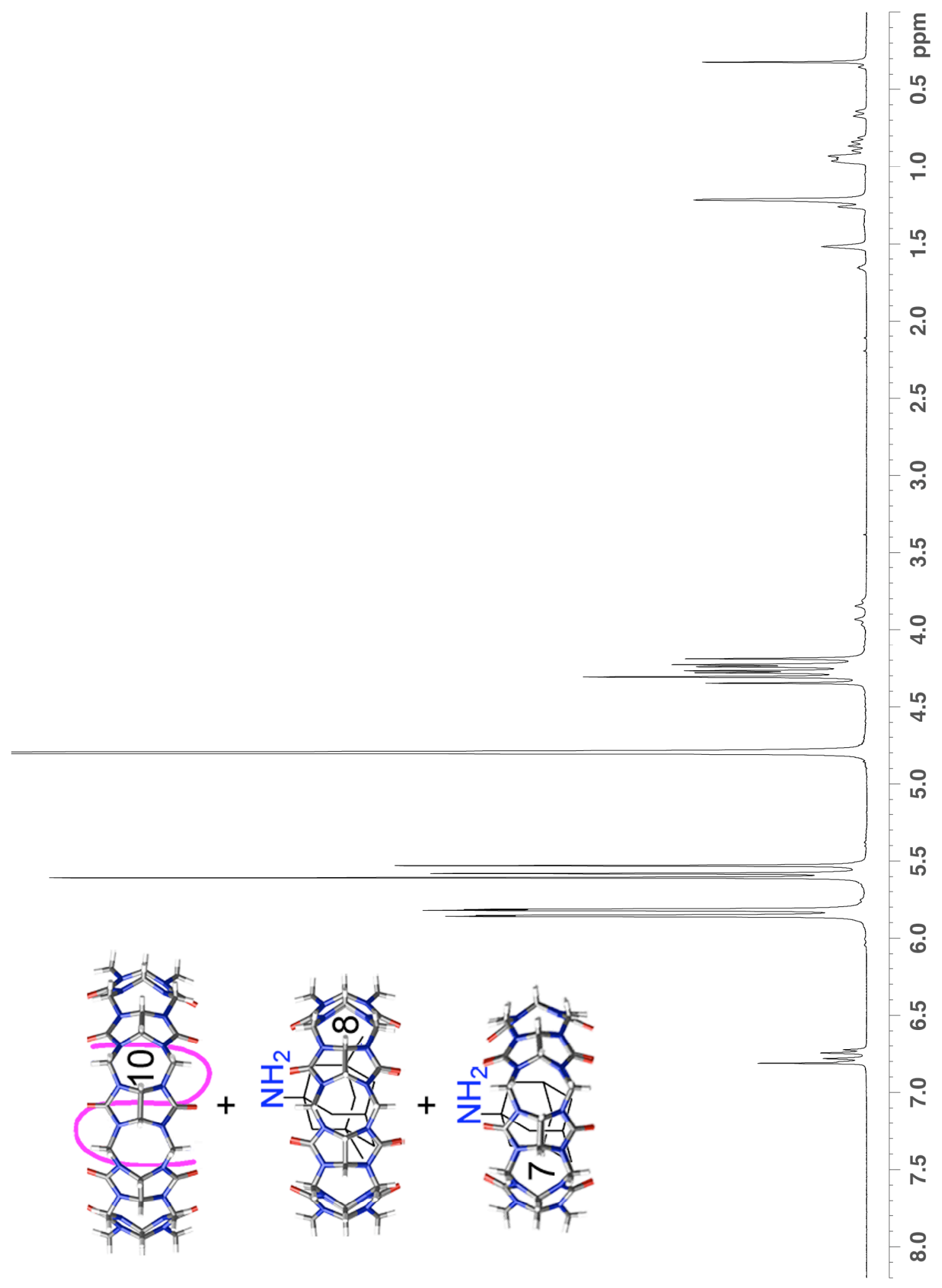

Figure S49. ${ }^{1} \mathrm{H}$ NMR spectra $\left(400 \mathrm{MHz}, \mathrm{D}_{2} \mathrm{O}, \mathrm{RT}\right)$ for a mixture of $\mathrm{CB}[10] \bullet \mathbf{3}+\mathrm{CB}[8] \bullet \mathbf{1 7}+2$ $\mathrm{CB}[7] \bullet 18$ prepared by sequential addition. 


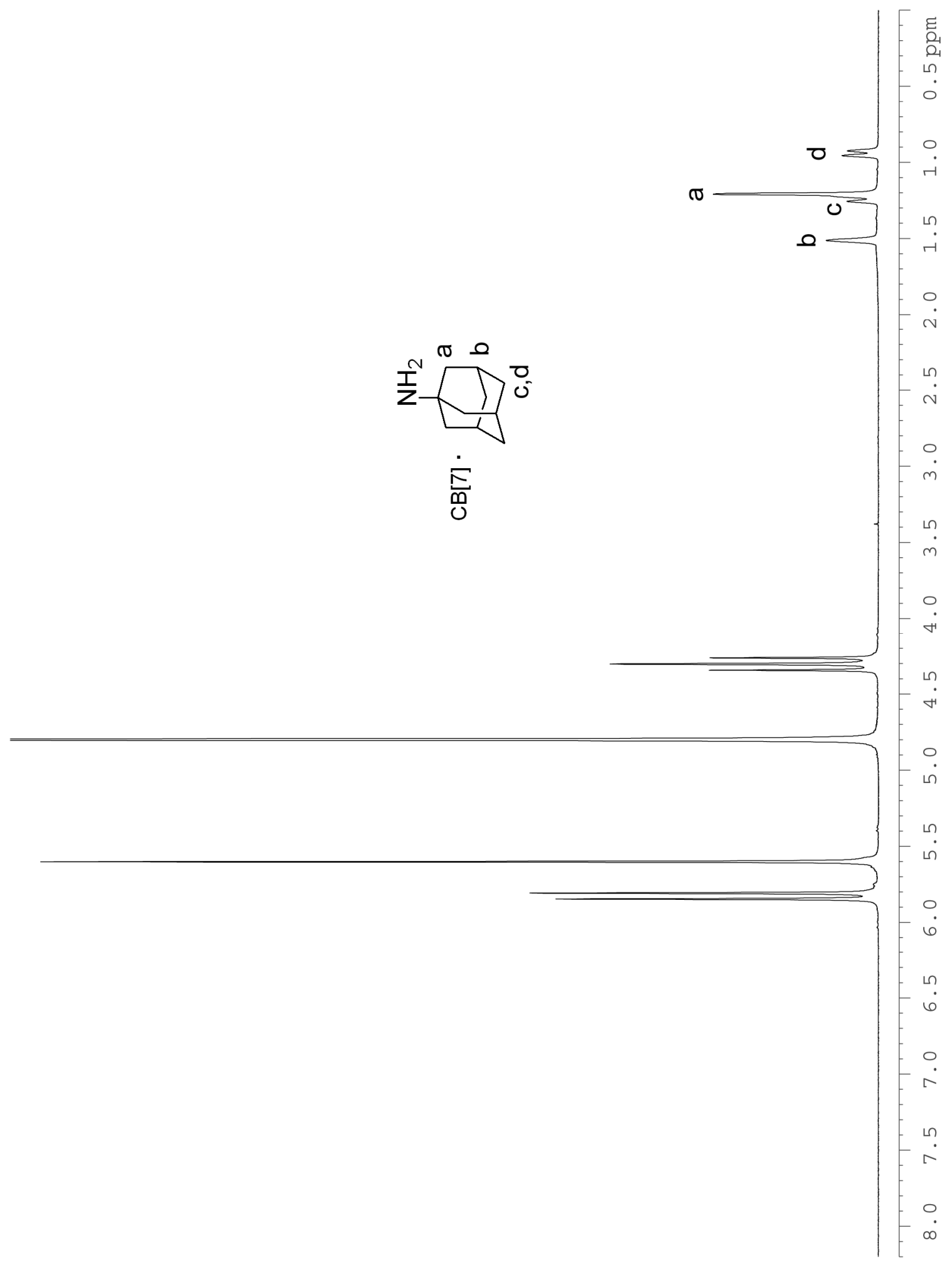

Figure S50. ${ }^{1} \mathrm{H}$ NMR spectra $\left(400 \mathrm{MHz}, \mathrm{D}_{2} \mathrm{O}, \mathrm{RT}\right)$ for $\mathrm{CB}[7] \bullet 18$. 


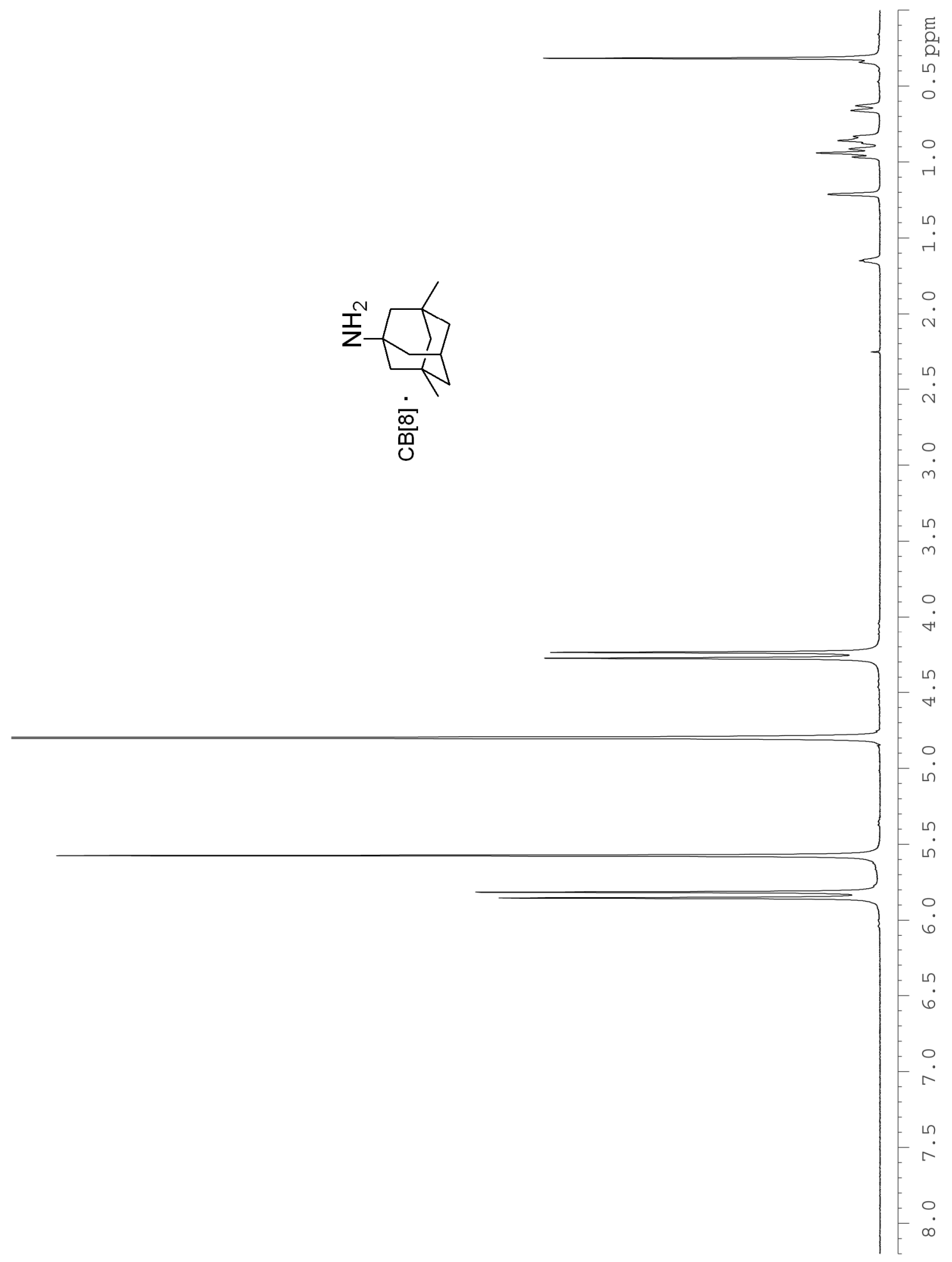

Figure S51. ${ }^{1} \mathrm{H}$ NMR spectra $\left(400 \mathrm{MHz}, \mathrm{D}_{2} \mathrm{O}, \mathrm{RT}\right)$ for $\mathrm{CB}[8] \bullet 17$. 


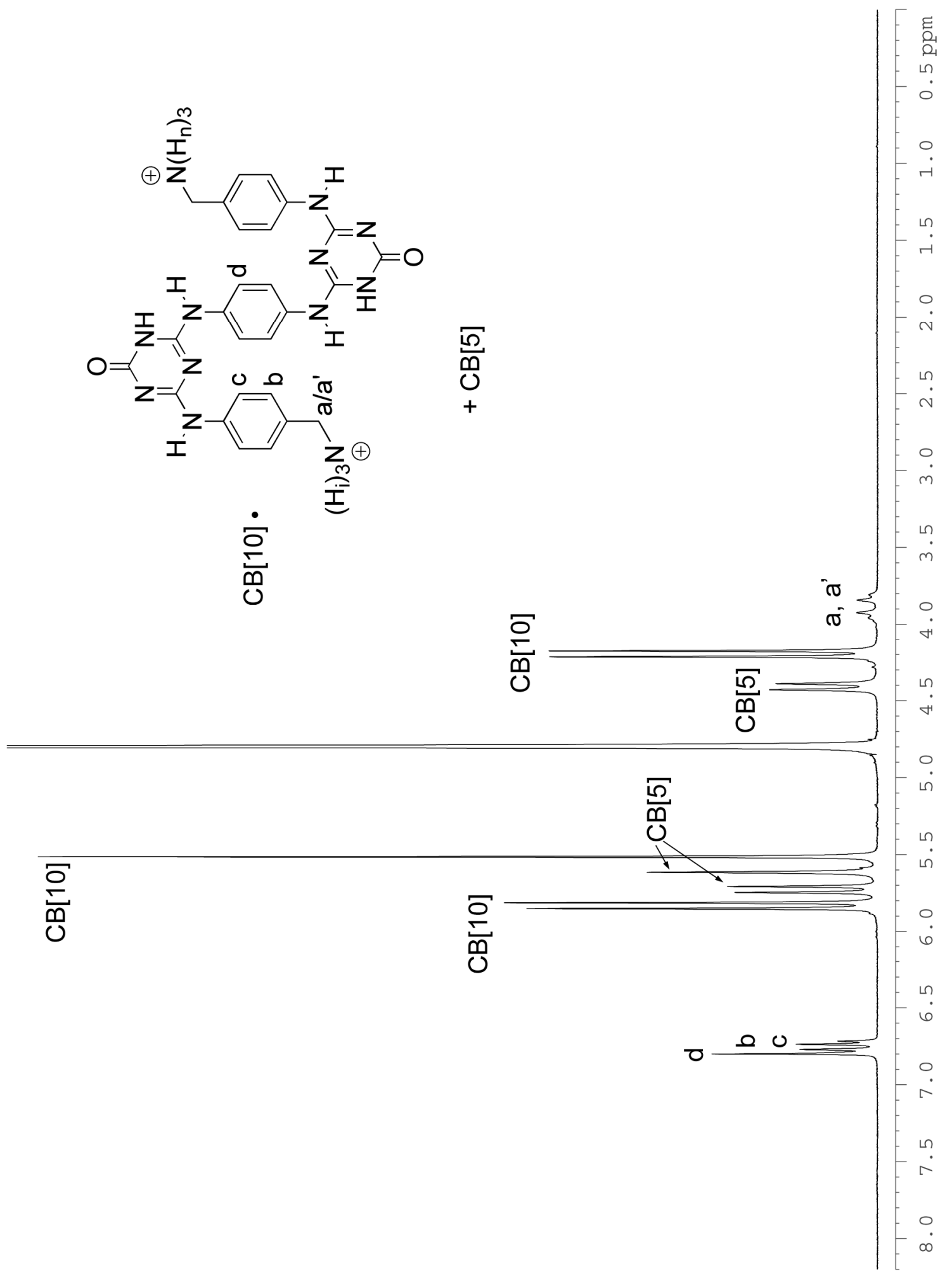

Figure S52. ${ }^{1} \mathrm{H}$ NMR spectra $\left(400 \mathrm{MHz}, \mathrm{D}_{2} \mathrm{O}, \mathrm{RT}\right)$ for $\mathrm{CB}[10] \cdot 3+\mathrm{CB}[5]$ prepared by the addition of $\mathbf{3}$ to $\mathrm{CB}[10] \cdot \mathrm{CB}[5]$. 


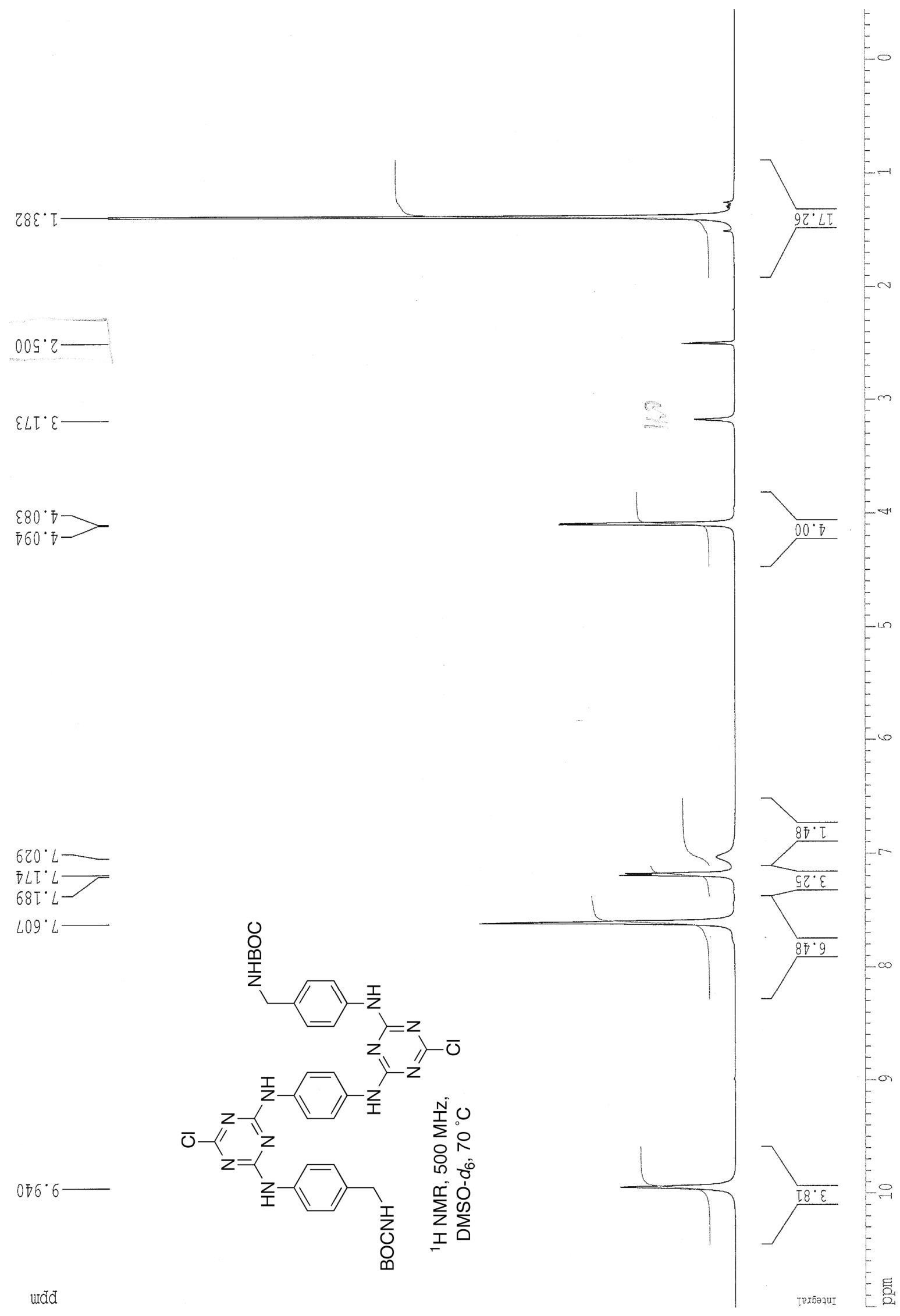




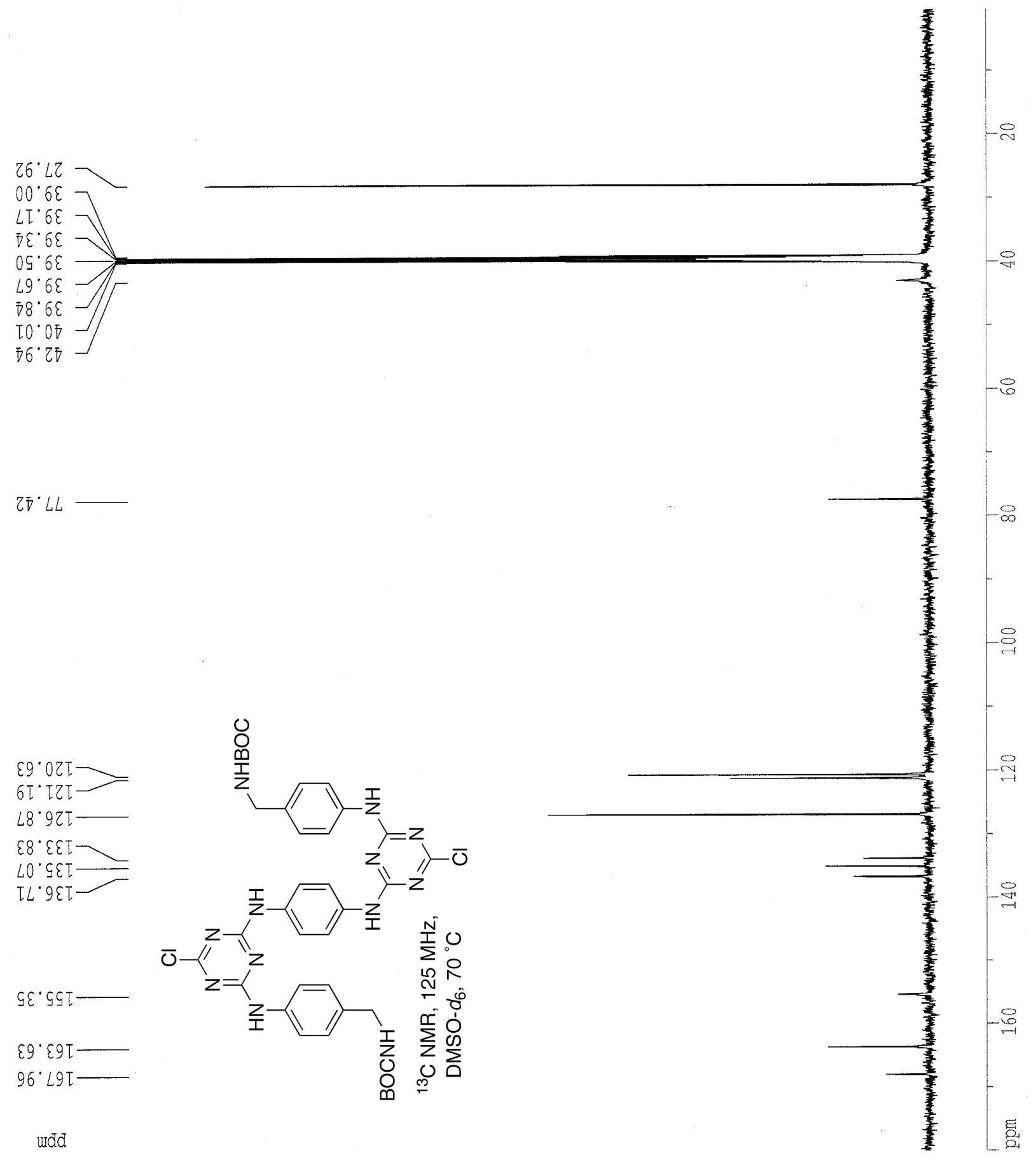




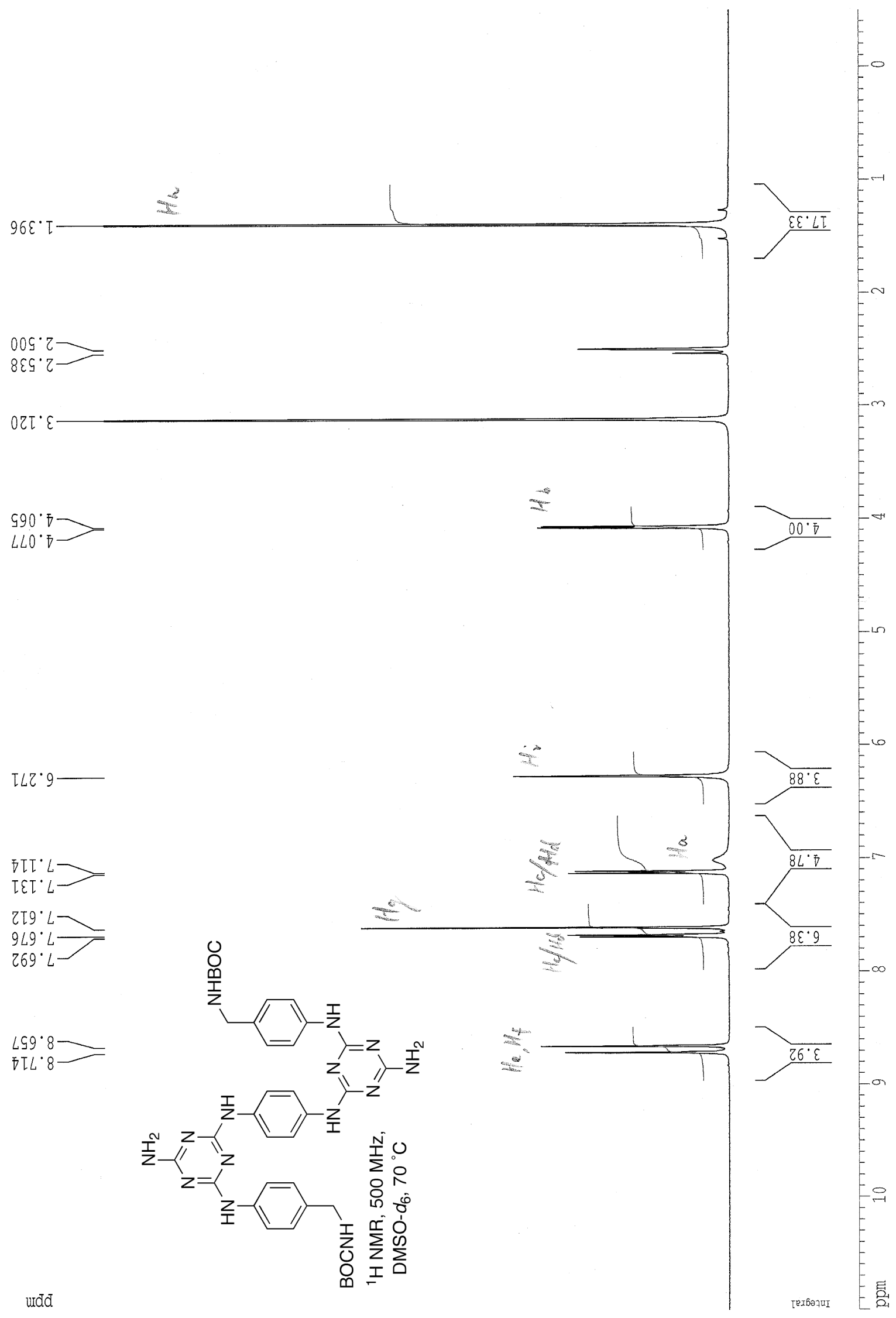




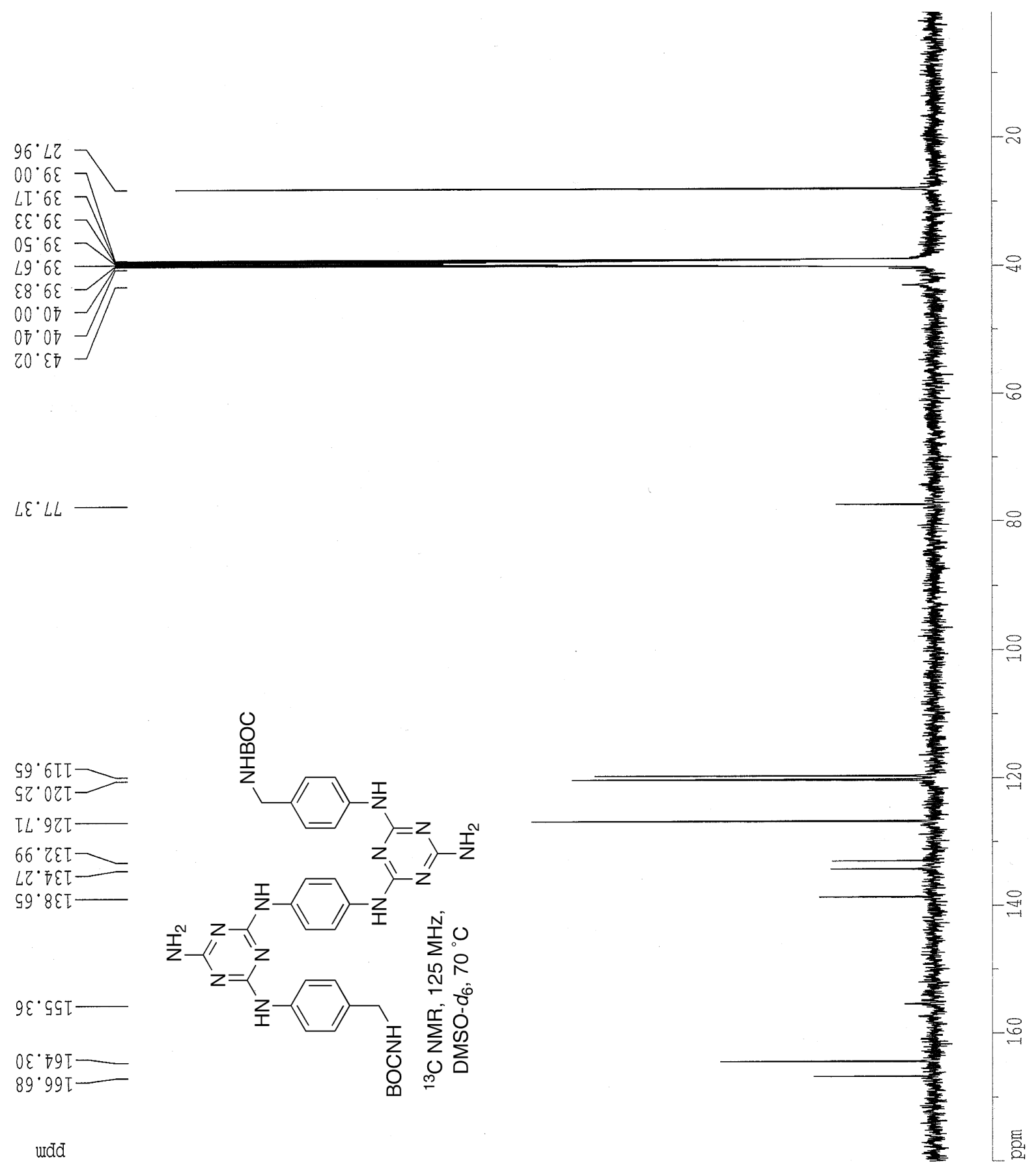




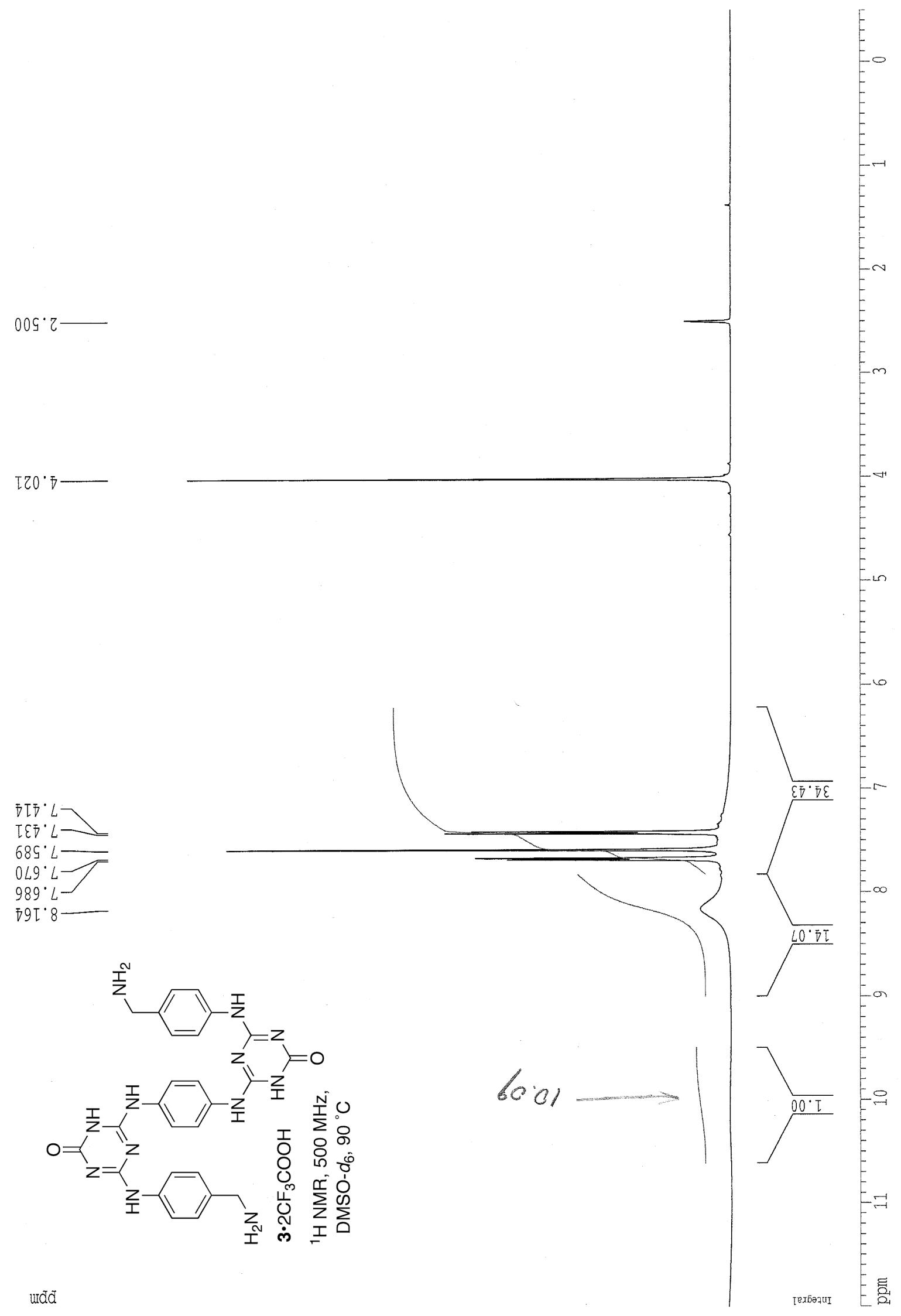




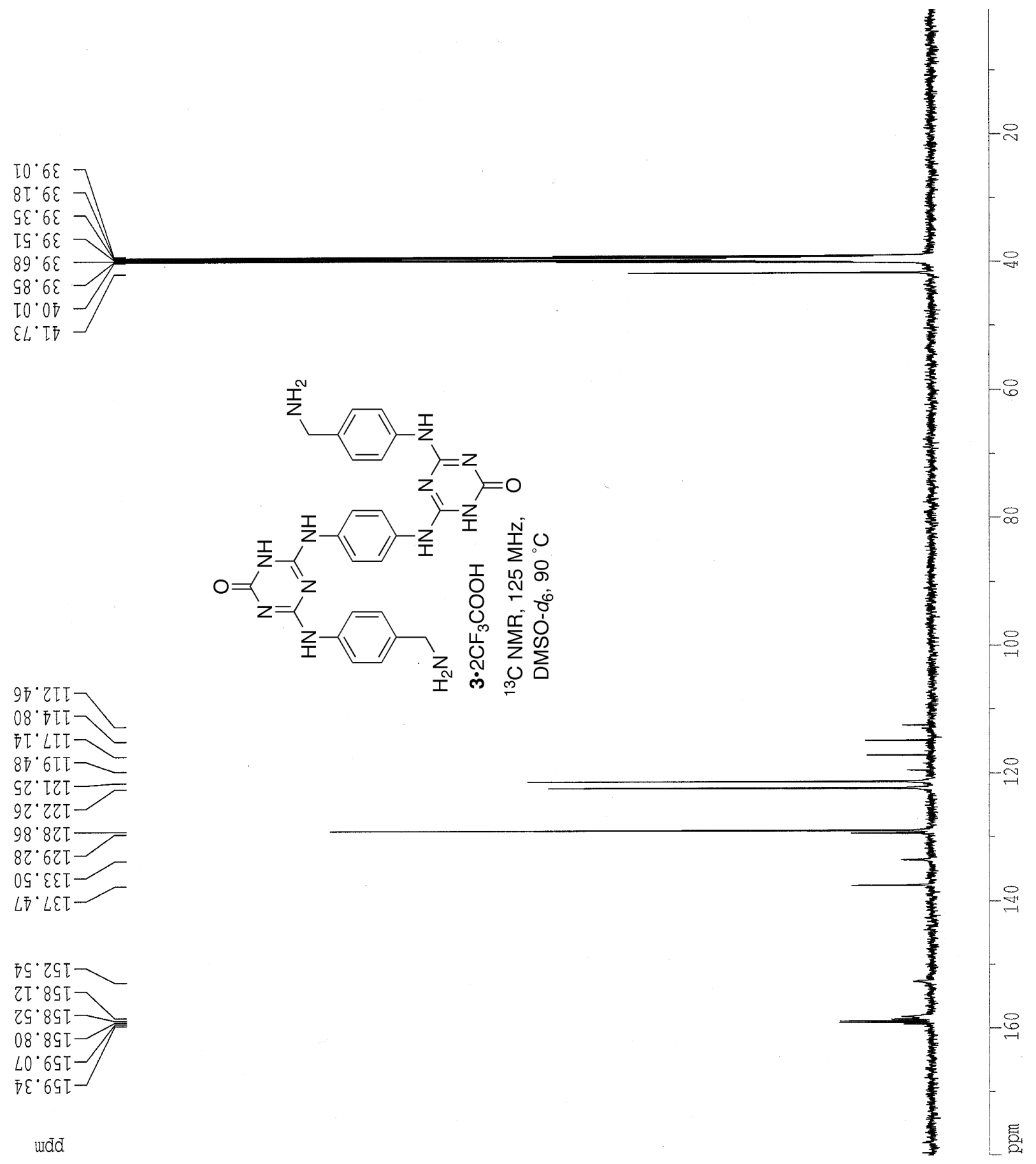




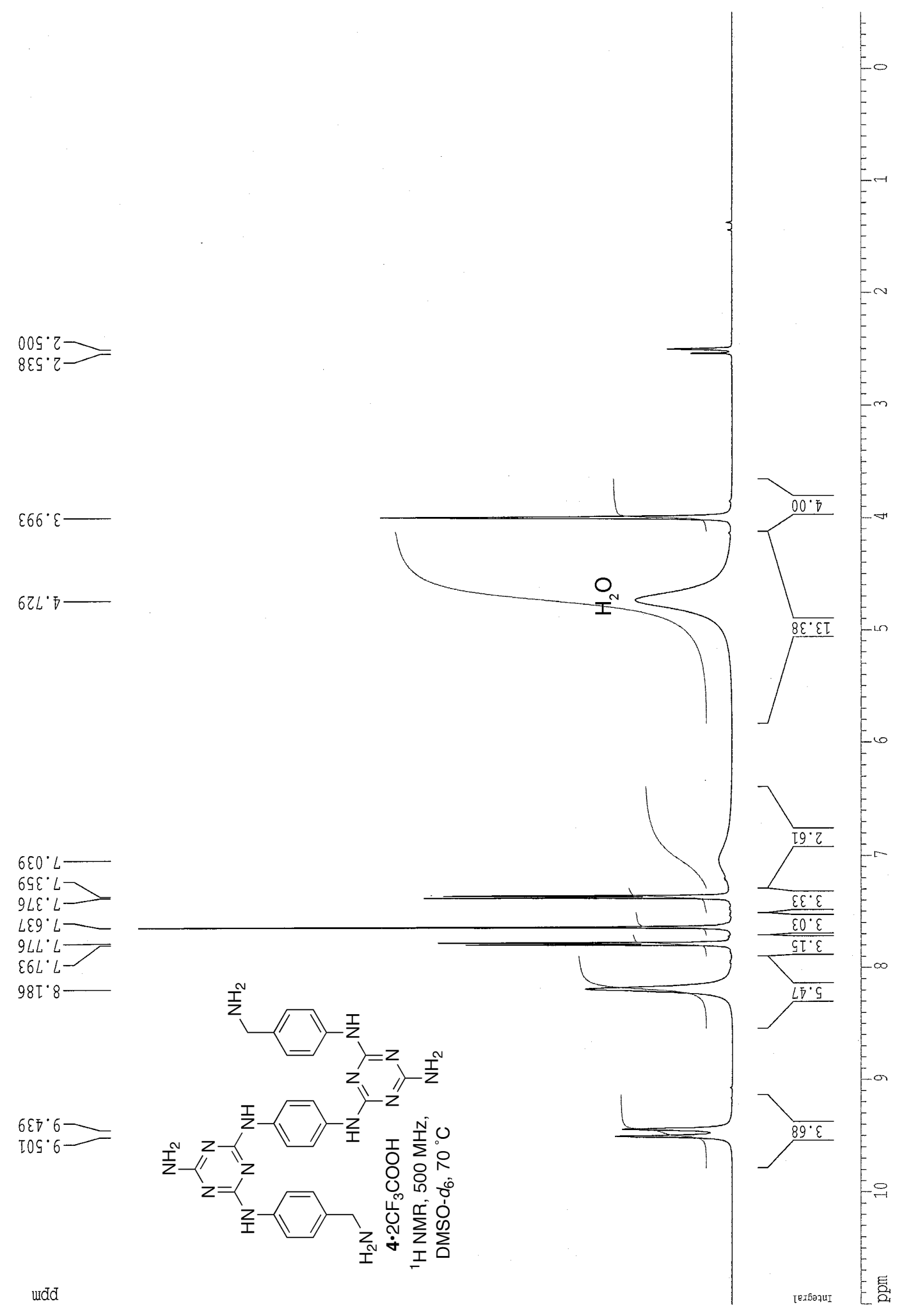




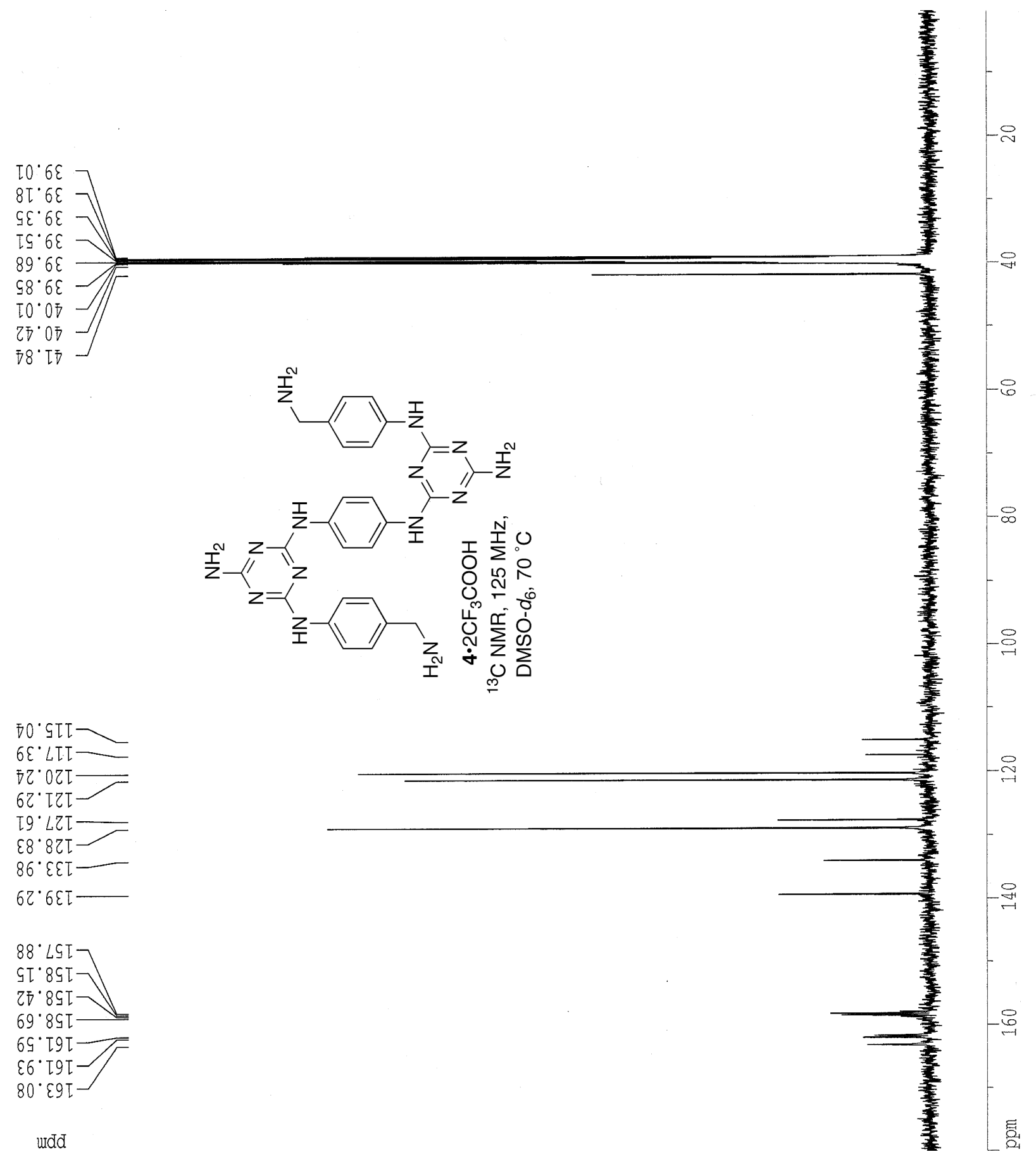




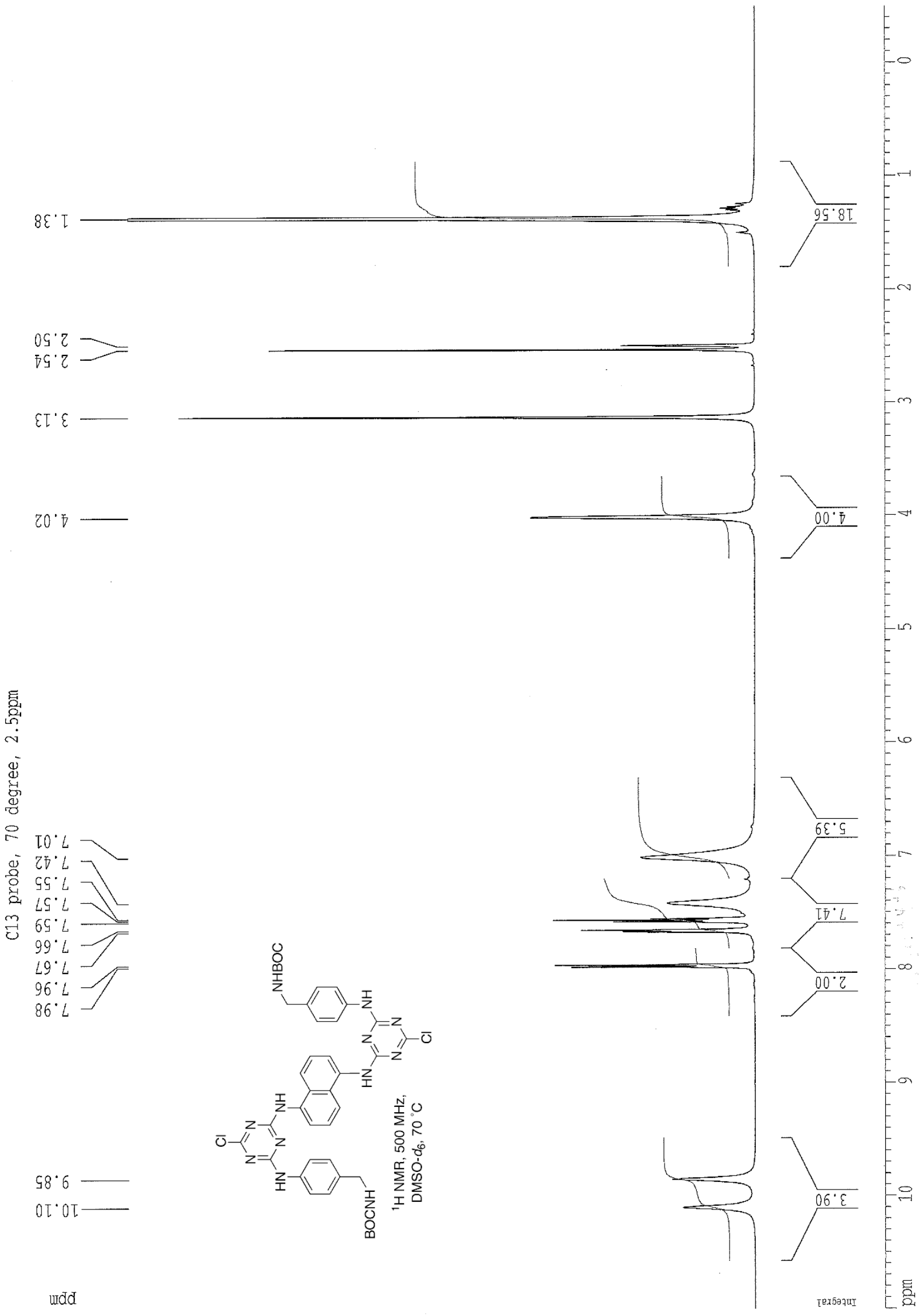




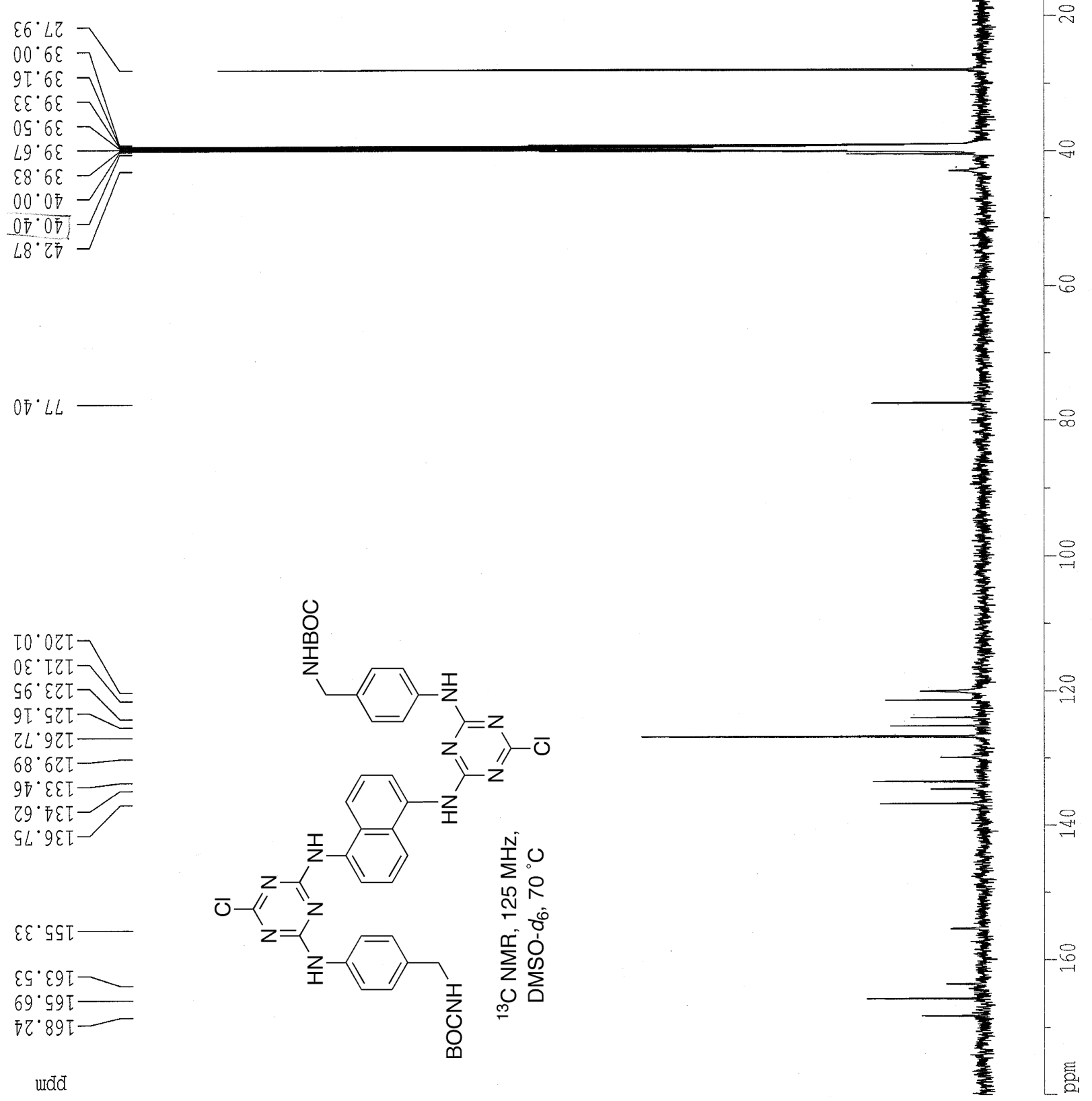




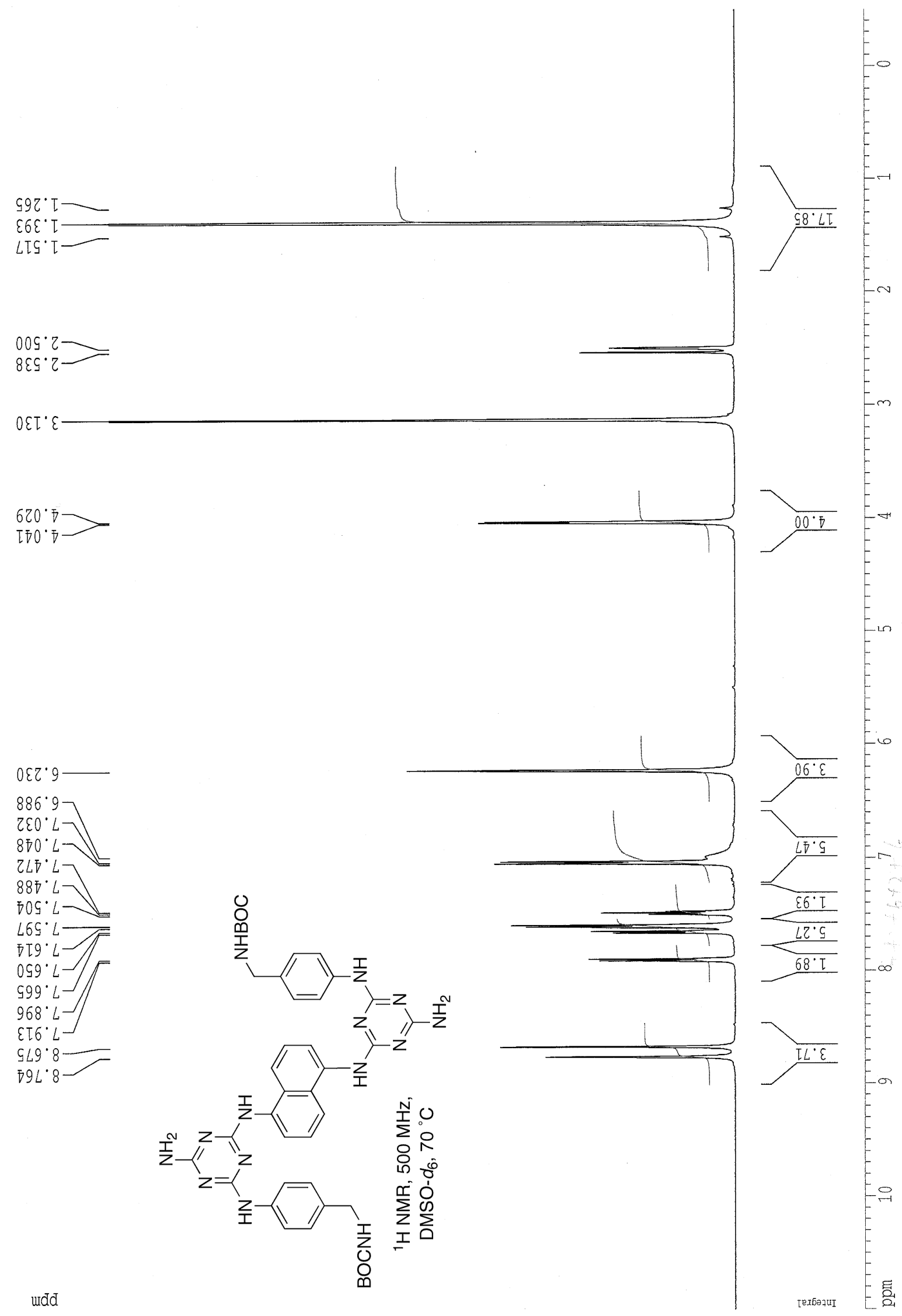




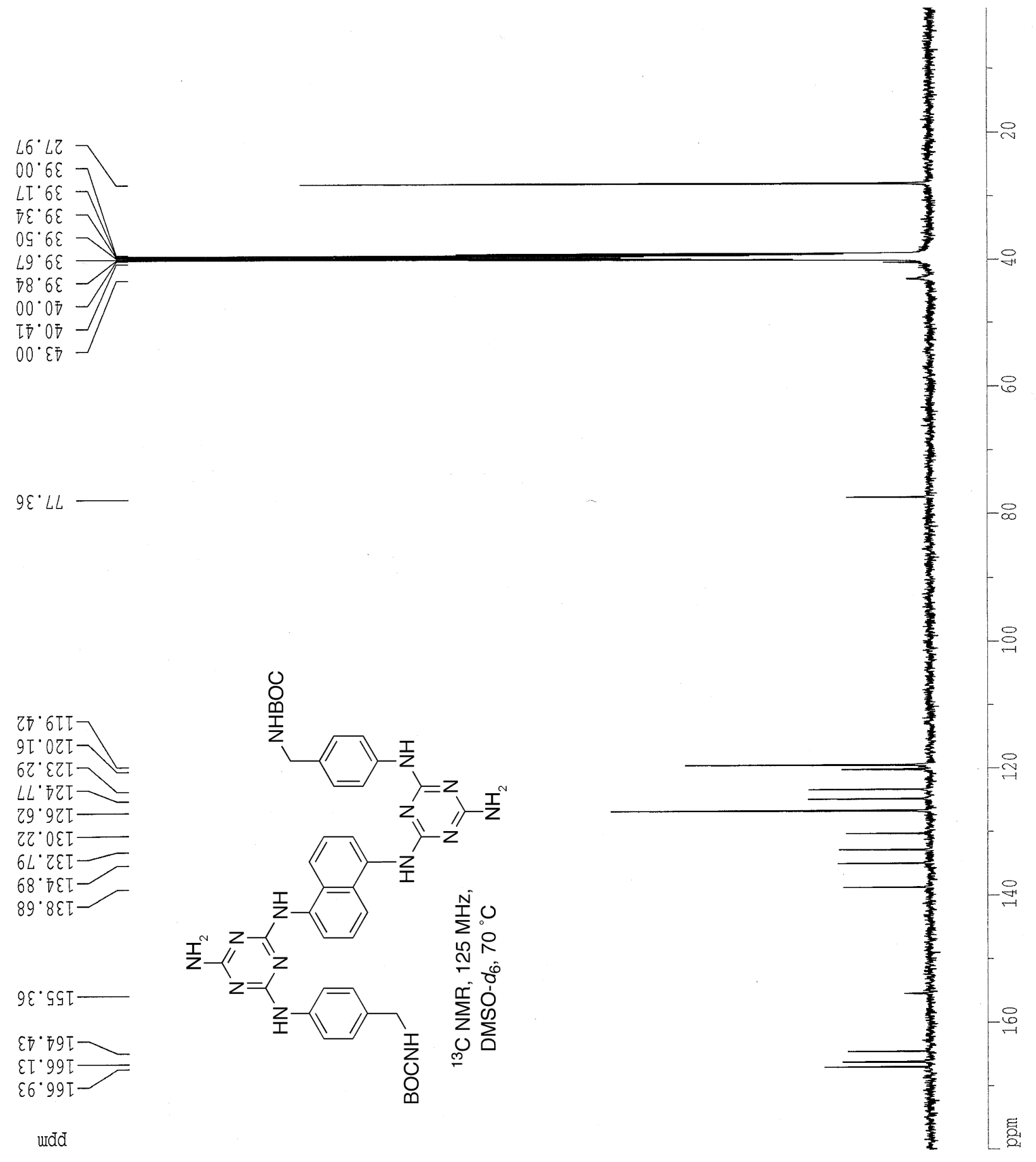




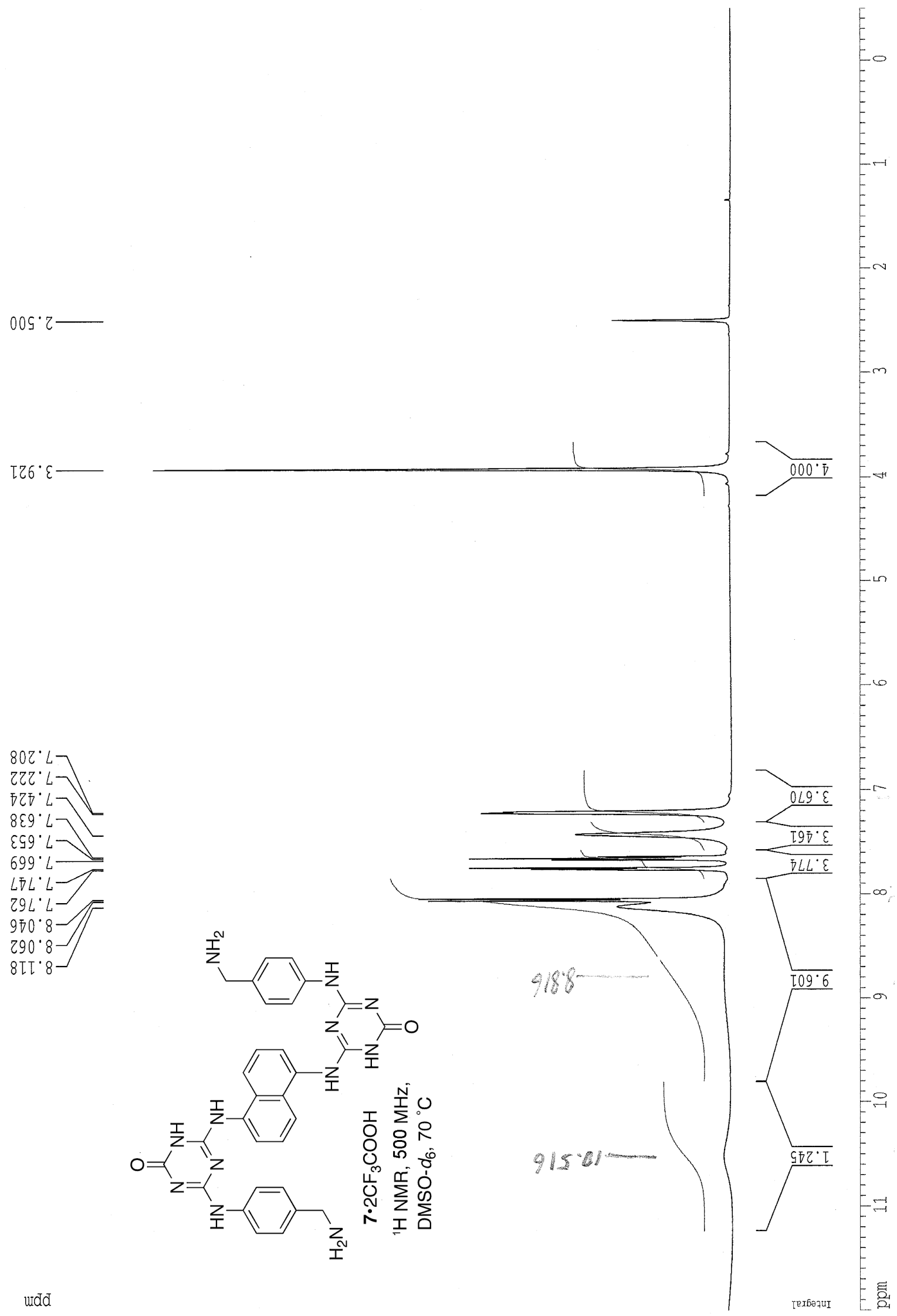




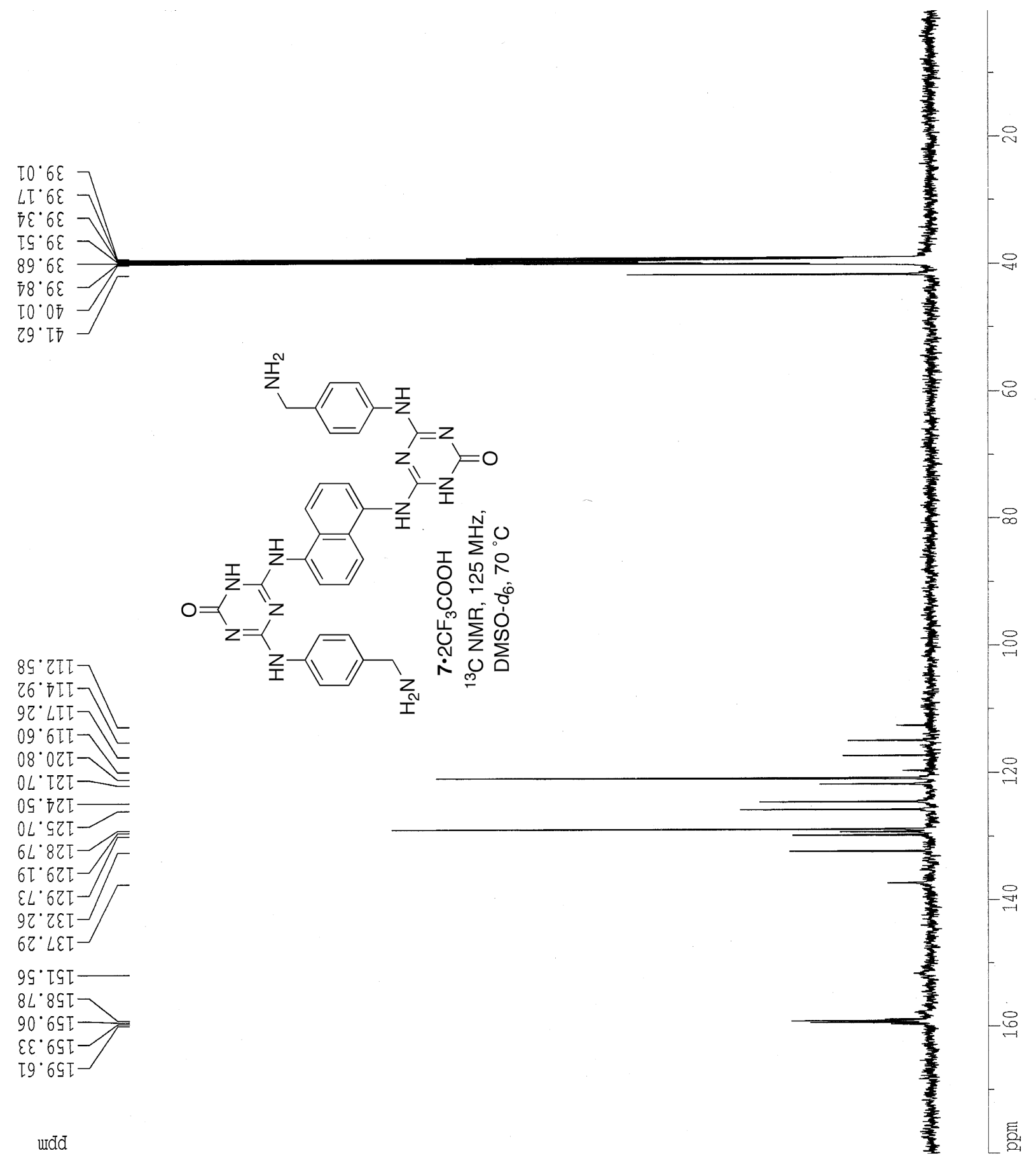


$005^{\circ} 2$

$L 76^{\circ} \varepsilon$

ZLE'

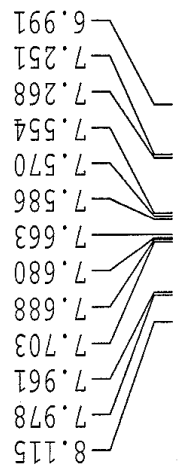

$807 \cdot 6$

$875 \cdot 6$

urdd

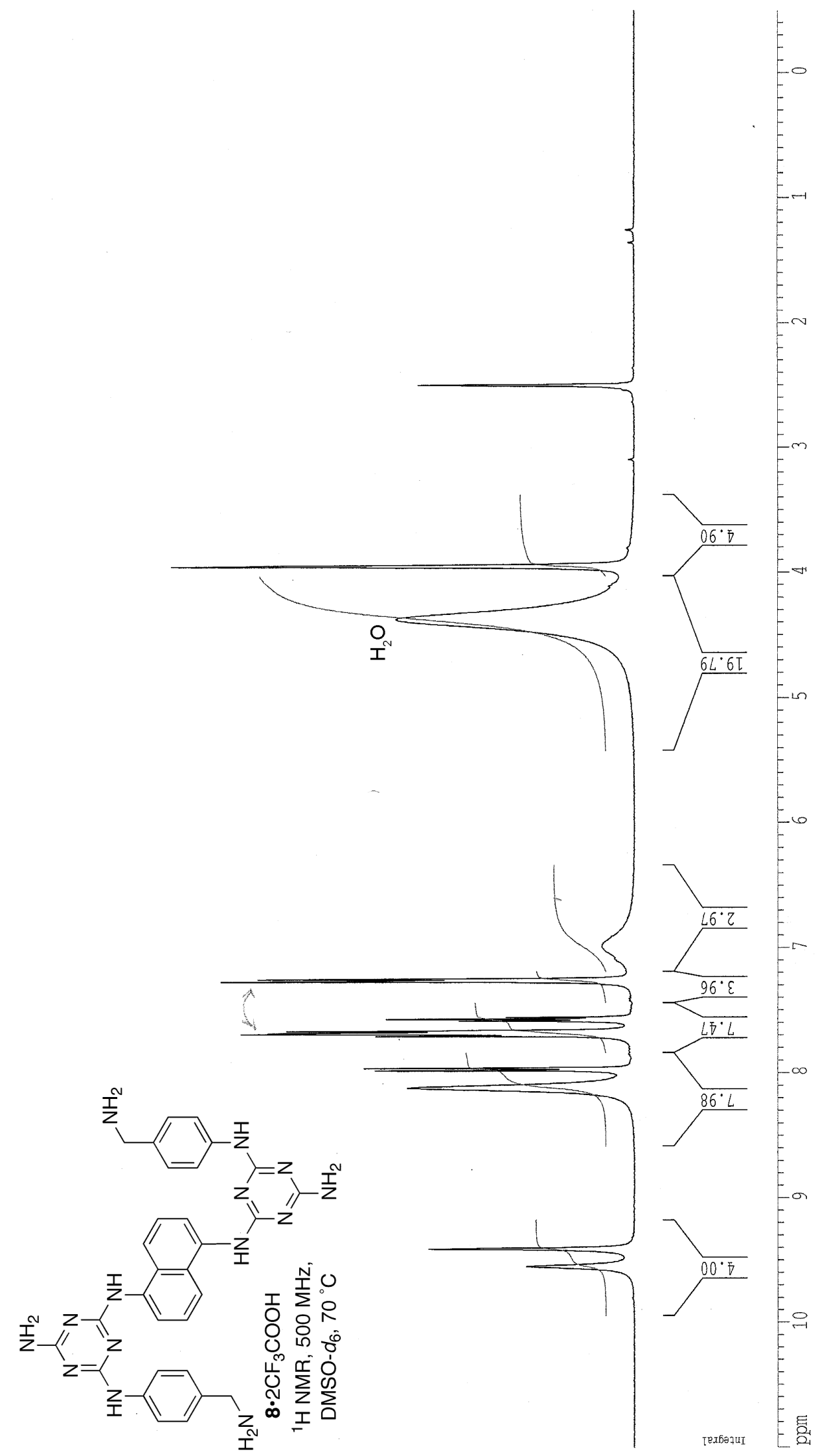




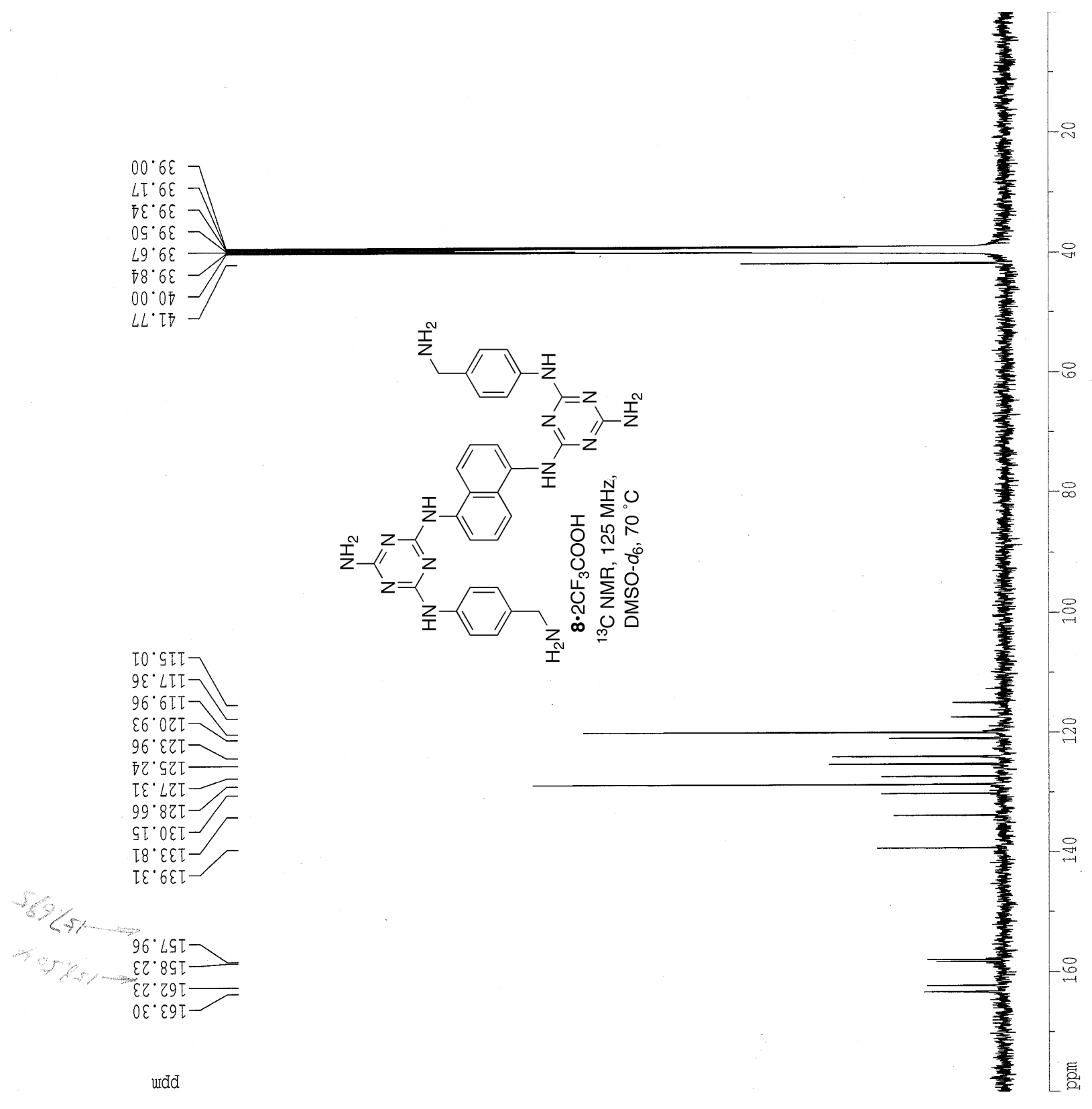




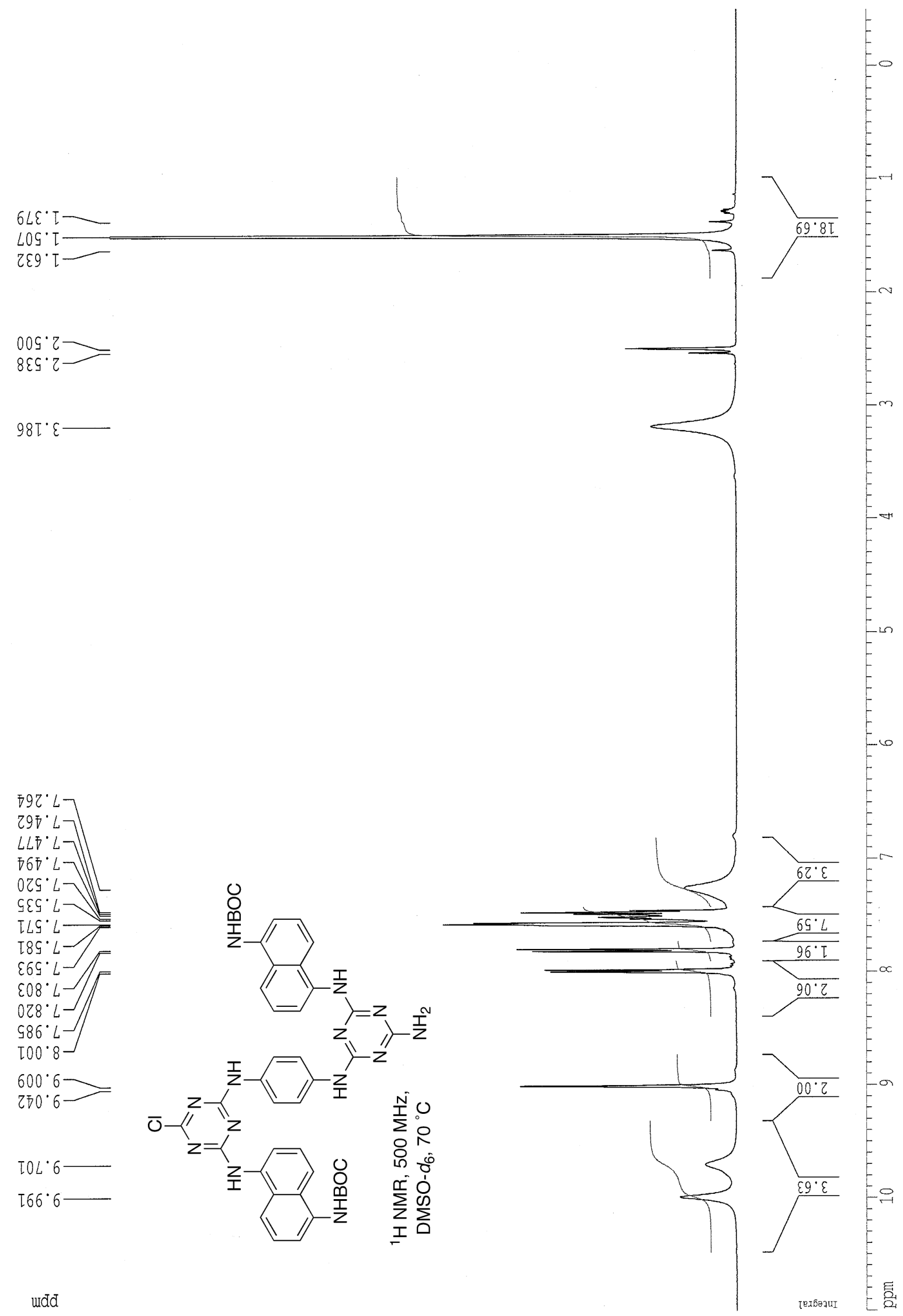




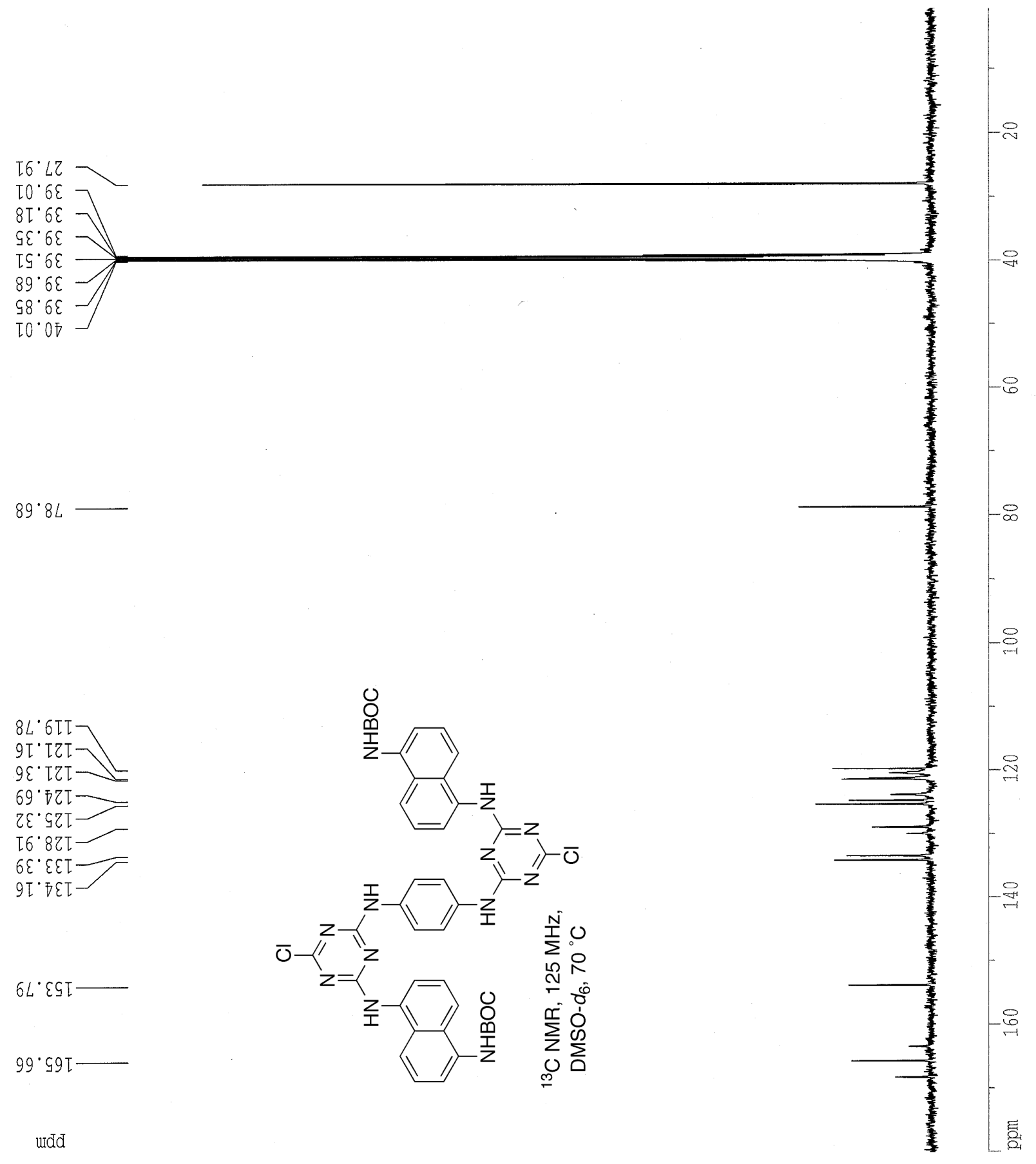




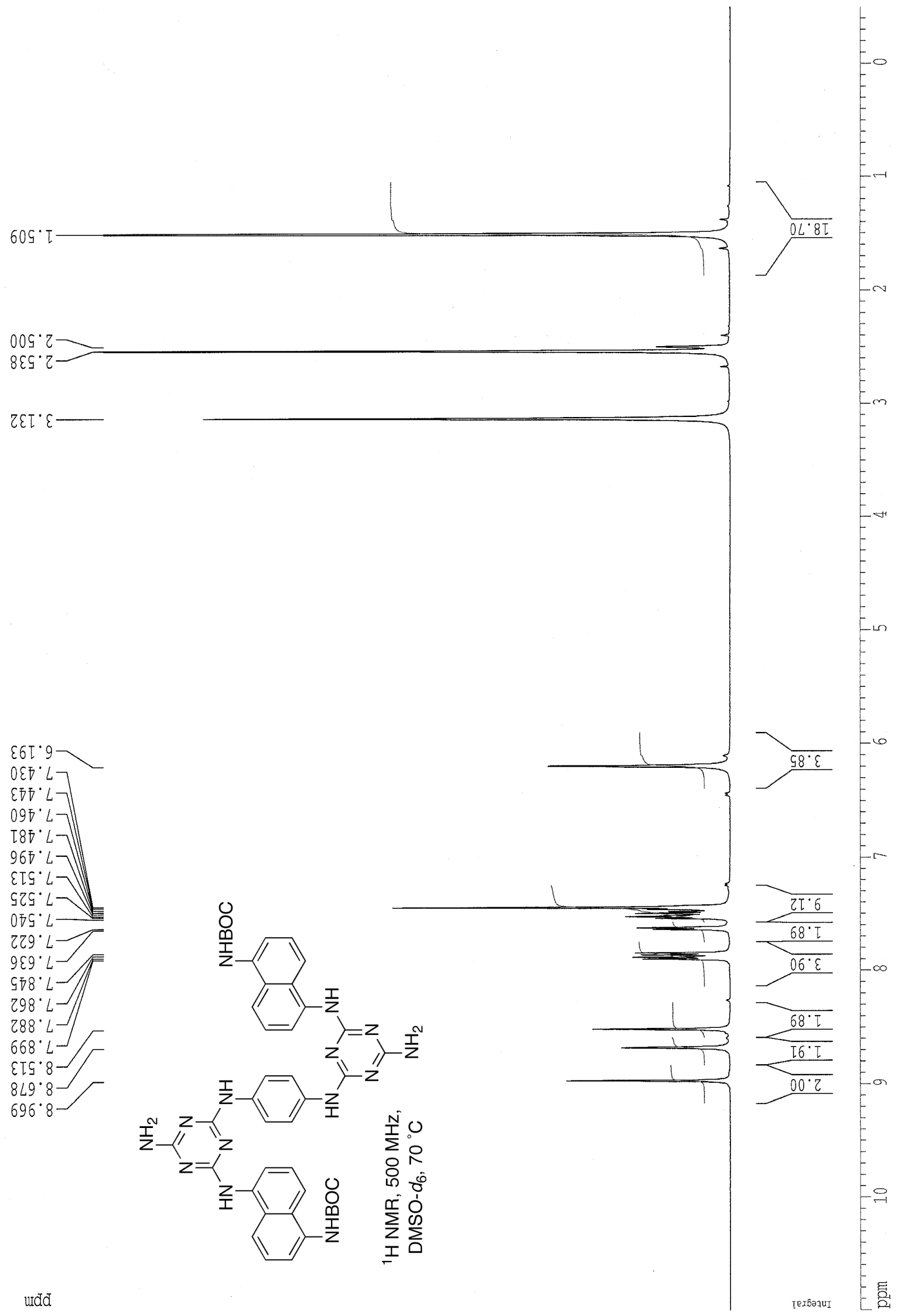




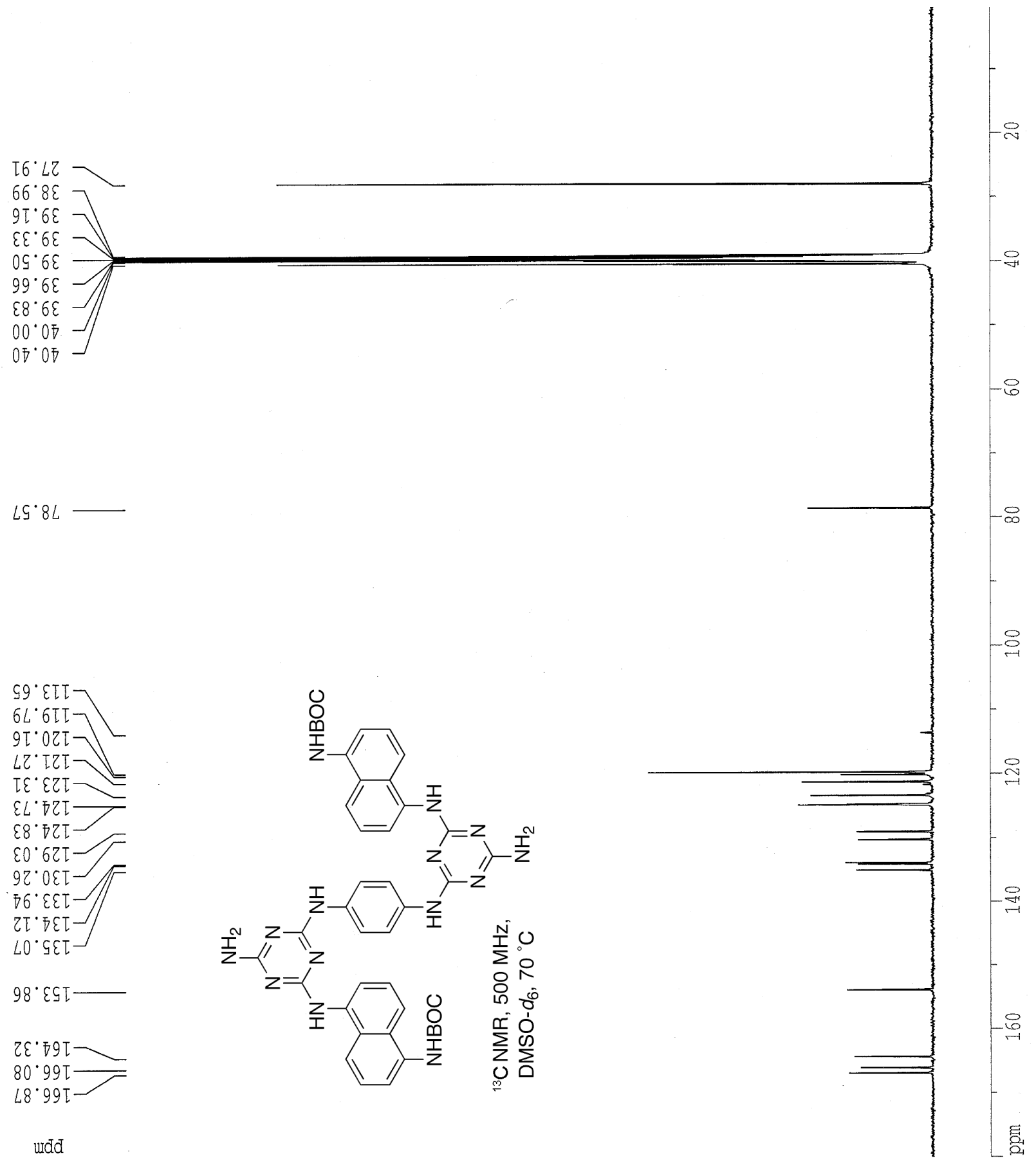


$0 s^{\circ} z=$

$\nabla G \cdot z$
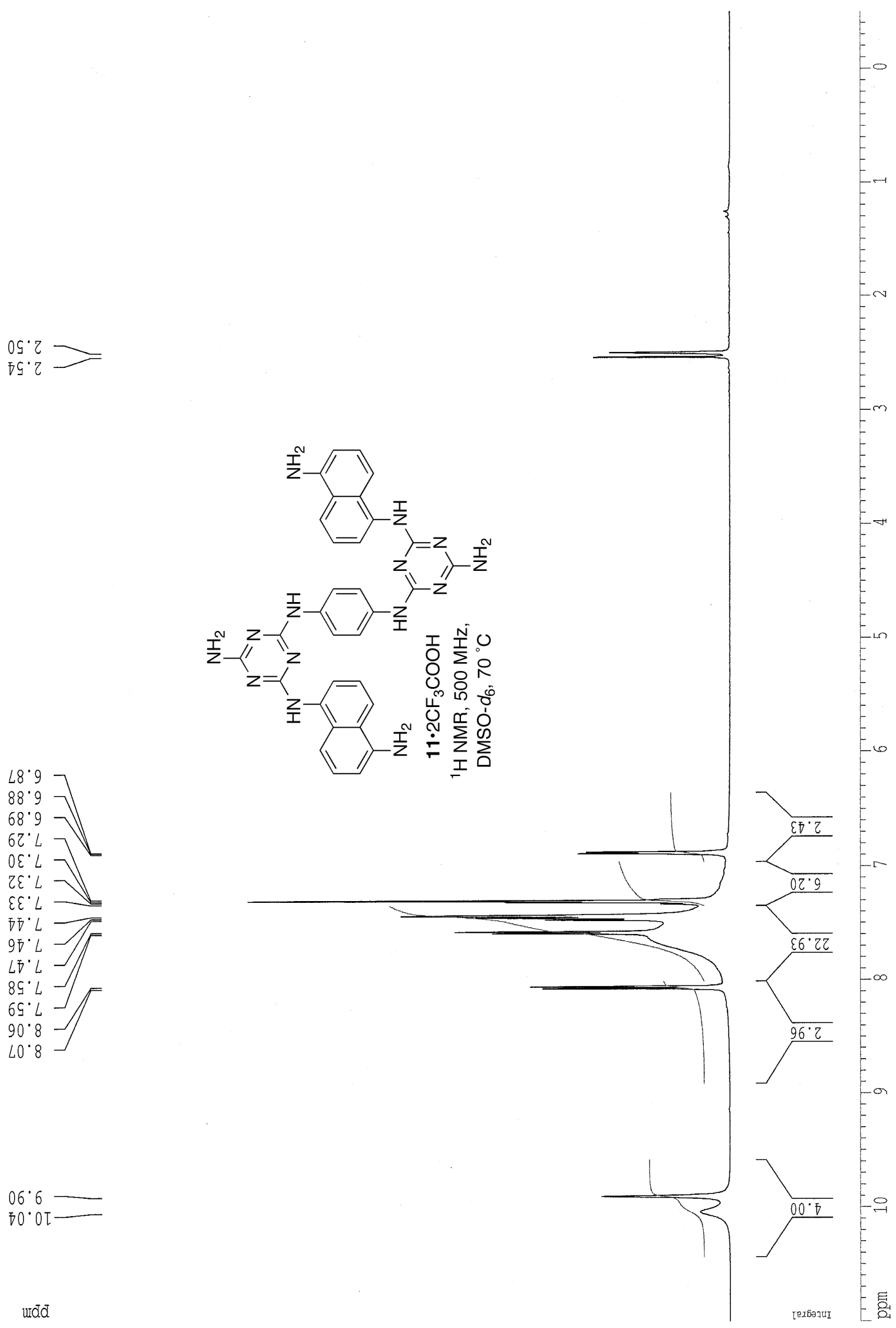

$06^{\circ} 6$

$70^{\circ} \mathrm{OT}$

ưd] 


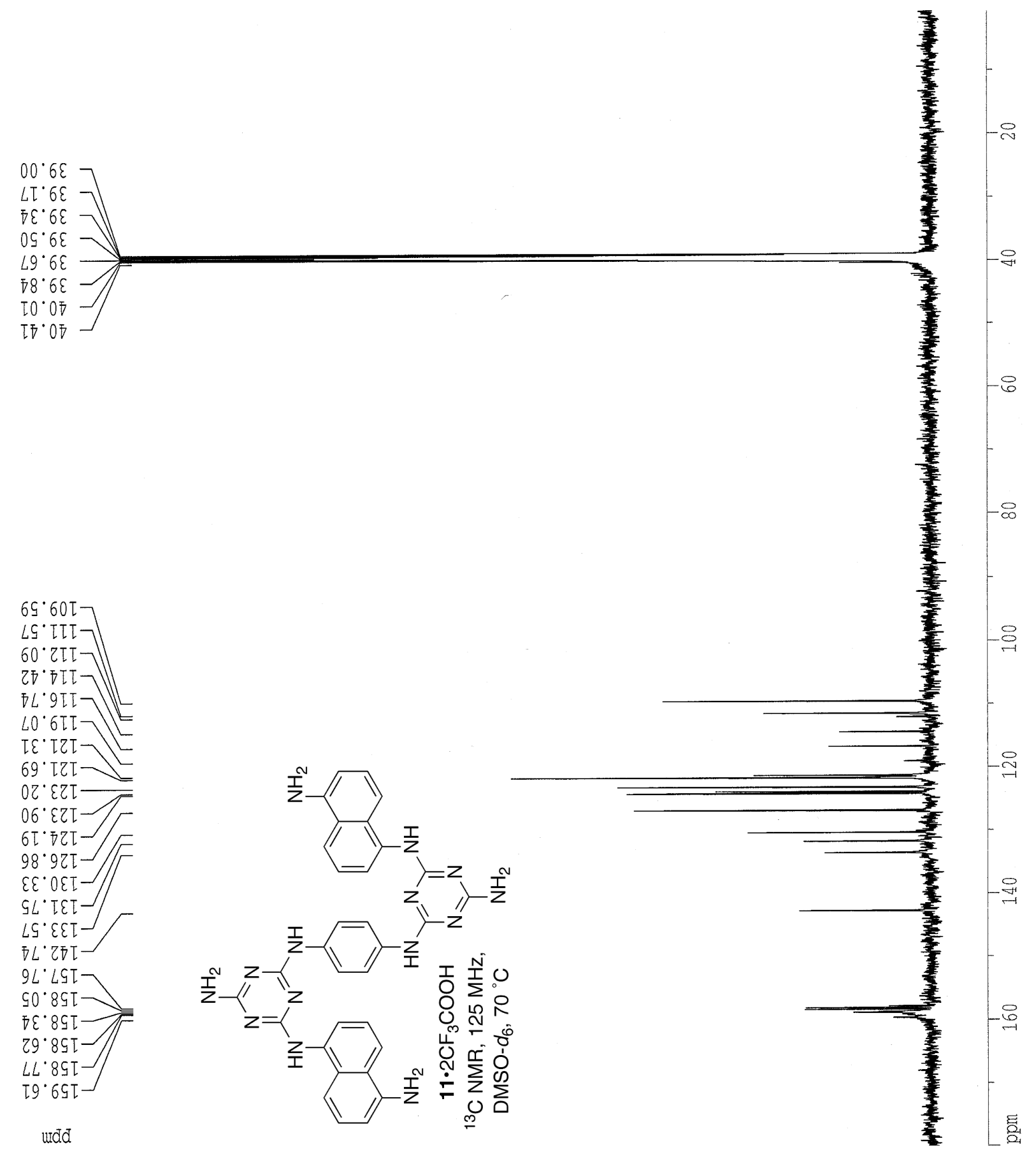

